\title{
Ford fundamental domains in symmetric spaces of rank one
}

\author{
Anke D. Pohl
}

Received: 2 July 2009 / Accepted: 13 December 2009 / Published online: 12 January 2010

(C) The Author(s) 2010. This article is published with open access at Springerlink.com

\begin{abstract}
We show the existence of isometric (or Ford) fundamental regions for a large class of subgroups of the isometry group of any rank one Riemannian symmetric space of noncompact type. The proof does not use the classification of symmetric spaces. All hitherto known existence results of isometric fundamental regions and domains are essentially subsumed by our work.
\end{abstract}

Keywords Ford fundamental domains - Isometric fundamental regions - Rank one symmetric spaces $\cdot$ Isometric spheres $\cdot$ Cygan metric

Mathematics Subject Classification (2000) $\quad 53 \mathrm{C} 35 \cdot 22 \mathrm{E} 40 \cdot 52 \mathrm{C} 22$

\section{Introduction}

Let $D$ be a rank one Riemannian symmetric space of noncompact type and denote its full group of Riemannian isometries by $G$. Suppose that $\Gamma$ is a subgroup of $G$. A subset $Y$ of $D$ is called a fundamental region for $\Gamma$ in $D$ if $Y$ is open, the translates of $Y$ by each two elements of $\Gamma$ are disjoint, and $D$ is covered by the family of $\Gamma$-translates of the closure of $Y$. If, in addition, $Y$ is connected, then it is called a fundamental domain for $\Gamma$ in $D$.

In this article, we show the existence of so-called isometric (or Ford) fundamental regions for a large class of subgroups of $G$, see Theorem 3.18, Corollary 3.20 and Proposition 4.38. In many situations, the fundamental regions will turn out to actually be fundamental domains (see Corollary 3.23 and Proposition 4.38). Moreover, in Sect. 4, we show that our results subsume all previously known existence results of isometric fundamental regions and domains.

Let $\Gamma$ be an admissible subgroup of $G$. The distinctive trait of an isometric fundamental region for $\Gamma$ is that it consists of two building blocks. One of them is a fundamental region $\mathcal{F}_{\infty}$ for the stabilizer group $\Gamma_{\infty}$ of $\infty$. The other one is the common part of the exteriors

A. D. Pohl $(\bowtie)$

Max-Planck-Institut für Mathematik, Vivatsgasse 7, 53111 Bonn, Germany

e-mail: pohl@mpim-bonn.mpg.de 
of all isometric spheres of $\Gamma$ (see Sect. 3 for a definition). Then the isometric fundamental region is the set

$$
\mathcal{F}:=\mathcal{F}_{\infty} \cap \bigcap_{g \in \Gamma \backslash \Gamma_{\infty}} \operatorname{ext} I(g),
$$

where ext $I(g)$ denotes the exterior of the isometric sphere $I(g)$ of $g \in \Gamma \backslash \Gamma_{\infty}$. These fundamental regions are of interest for several applications. For example, they reflect the geometry of $\Gamma$ in a way particularly adjusted to the needs of the construction of a symbolic dynamics for the geodesic flow on the orbifold $\Gamma \backslash D$ (see [19]).

Our proof of the existence of isometric fundamental regions does not use the classification of rank one Riemannian symmetric spaces of noncompact type. This was made possible by the classification-free constructions of all these spaces provided by Cowling et al. in [6] and [7], respectively, by Korányi and Ricci in [15] and [16]. These constructions appear to be the first ones which are uniform both on the level of spaces and on the level of isometry groups. The approach in [6] and [7] is intimately connected with the restricted root space decomposition of the Lie algebra of the isometry group of the symmetric space, and hence it is the method of choice for considerations of algebraic nature. In contrast, the construction in [16] and [15] reflects the geometric side of the spaces. Both constructions are amazingly easy to work with. Moreover, one can effortless switch from one construction to the other and translate insights and advantages from one model to the other. We recall both constructions in Sect. 2.

Using their work we provide a uniform definition of the notion of an isometric sphere and its exterior in Sect. 3. The uniformity on the level of isometry groups then allows to stick to a classification-free treatment of the isometric spheres, which finally results in a classification-free proof of the existence of isometric fundamental regions.

For real, complex and quaternionic hyperbolic spaces, there already exist several (different and also non-equivalent) definitions of isometric spheres in the literature, e.g., in [10] for the hyperbolic plane, in [13] for the upper half plane model and the disk model of twodimensional real hyperbolic space, in [17] for three-dimensional real hyperbolic space, in [1] and [2] for real hyperbolic spaces of arbitrary dimension, in [11,18] and [12] for complex hyperbolic spaces, and in [14] for quaternionic hyperbolic spaces. Moreover, for certain subgroups of the isometry group of real and complex hyperbolic spaces, the existence of isometric fundamental regions was already known. More precisely, Apanasov [1] and [2] provides the hitherto most general treatment of groups acting on real hyperbolic spaces. In [10], Ford investigates the case of the hyperbolic plane. However, his definition of fundamental region is not equivalent to our definition. Therefore, his result cannot be compared to our one. Groups acting on complex hyperbolic spaces are considered by Kamiya in [12]. In Sect. 4, we will investigate which definitions of isometric spheres are subsumed by our uniform one, and we will show that the known isometric fundamental regions are special cases of Theorem 3.18.

Throughout we will use the following notation. If $T$ is a topological space and $U$ a subset of $T$, then the closure of $U$ is denoted by $\bar{U}$ or $\operatorname{cl}(U)$ and its boundary is denoted by $\partial U$. Moreover, we write $U^{\circ}$ for the interior of $U$. The complement of $U$ in $T$ is denoted by $\complement U$ or $T \backslash U$.

For two arbitrary sets $A$ and $B$, the complement of $B$ in $A$ is written as $A \backslash B$. If $\sim$ is an equivalence relation on $A$, then $A / \sim$ denotes the set of equivalence classes. Likewise, if $\Gamma$ a group acting on $A$, then we write $A / \Gamma$ for the space of right cosets. Moreover, if $p$ is a point of $A$, then $\Gamma_{p}$ denotes the stabilizer group $\{g \in \Gamma \mid g p=p\}$ of $p$ in $\Gamma$. 


\section{Classification-free constructions}

The basic objects of the classification-free construction of rank one Riemannian symmetric spaces of noncompact type in [6] and [7] are so-called $H$-type (Heisenberg type) algebras. In contrast, Korányi and Ricci [15] and [16] construct these symmetric spaces from so-called $J^{2} C$-module structures.

In this section, we introduce these notions and we briefly recall the two constructions. Mostly we duplicate, for the convenience of the reader, material from their work. Nevertheless, in parts it can be considered as complementary, e. g., Lemma 2.2 on the (non-)uniqueness of decompositions of $\mathrm{H}$-type algebras, the notion of ordered decompositions, and a refined definition of an isomorphism between $H$-type algebras, respectively, between $C$-module structures (which corrects a minor inaccuracy). Moreover, we prove in detail that the Cayley transform is an isometry, and that the group $M$ given in [6] and [7] and that in [15] and [16] are indeed the same.

All omitted proofs can be found in [6] or [7] for statements in the language of $H$-type algebras, and in [15] or [16] for those in the language of $J^{2} C$-modules. As long as no confusion can arise, each inner product is denoted by $\langle\cdot, \cdot\rangle$ and its associated norm by $|\cdot|$.

\section{1 $H$-type algebras and the $J^{2}$-condition}

A vector space is called Euclidean if it is a finite-dimensional real vector space endowed with an inner product. A Lie algebra is called Euclidean if, in addition to being a Lie algebra, it is a Euclidean vector space. For a vector space $\mathfrak{v}$ let $\operatorname{End}_{\mathrm{vs}}(\mathfrak{v})$ denote the group and vector space of endomorphisms of $\mathfrak{v}$. If $\mathfrak{v}$ carries additional structures, then the elements of $\operatorname{End}_{\mathrm{vs}}(\mathfrak{v})$ are not required to be compatible with these structures. In particular, if $\mathfrak{v}$ is Euclidean, then $\varphi \in \operatorname{End}_{\mathrm{vs}}(\mathfrak{v})$ need not be orthogonal.

Definition 2.1 Let $\mathfrak{n}$ be a Euclidean Lie algebra. Then $\mathfrak{n}$ is said to be an H-type algebra if

(H1) there are two subvector spaces $\mathfrak{v}, \mathfrak{z}$ of $\mathfrak{n}$ (each of which may be trivial) such that

$$
[\mathfrak{n}, \mathfrak{z}]=\{0\}, \quad[\mathfrak{n}, \mathfrak{n}] \subseteq \mathfrak{z},
$$

and $\mathfrak{n}$ is the orthogonal direct sum of $\mathfrak{z}$ and $\mathfrak{v}$,

(H2) for all $X \in \mathfrak{v}$, all $Z \in \mathfrak{z}$ we have

$$
|J(Z) X|=|Z| \cdot|X|
$$

where $J: \mathfrak{z} \rightarrow \operatorname{End}_{\mathrm{vs}}(\mathfrak{v})$ is the $\mathbb{R}$-linear map defined by

$$
\langle J(Z) X, Y\rangle=\langle Z,[X, Y]\rangle
$$

for all $X, Y \in \mathfrak{v}$, all $Z \in \mathfrak{z}$. The map $J$ is well-defined and unique by Riesz' Representation Theorem (or its finite-dimensional counterpart).

If (H1) holds, then $\mathfrak{n}$ is either abelian or two-step nilpotent. In the first case we call $\mathfrak{n}$ degenerate, in the second non-degenerate.

The construction of a symmetric space from an $H$-type algebra $\mathfrak{n}$ depends on the choice of $\mathfrak{z}$ and $\mathfrak{v}$ in the decomposition $\mathfrak{z} \oplus \mathfrak{v}$ of $\mathfrak{n}$. We call the pair $(\mathfrak{z}, \mathfrak{v})$ an ordered decomposition of $\mathfrak{n}$. The following lemma shows that the ordered decomposition is unique unless $\mathfrak{n}$ is non-trivial and abelian (which precisely is the reason for calling abelian $H$-type algebras degenerate). It will turn out that both possible ordered decompositions of a non-trivial abelian $H$-type algebra give rise to the same symmetric space, but in different models. Nevertheless, 
this non-uniqueness calls for a careful notion of isomorphisms between $H$-type algebras, which we will discuss after the lemma.

We denote the center of a Lie algebra $\mathfrak{g}$ by $Z(\mathfrak{g})$.

Lemma 2.2 Let $\mathfrak{n}$ be an H-type algebra. If $\mathfrak{n}$ is non-abelian or $\mathfrak{n}=\{0\}$, then the ordered decomposition $(\mathfrak{z}, \mathfrak{v})$ of $\mathfrak{n}$ is unique. In this case, we have $\mathfrak{z}=Z(\mathfrak{n})$ and $\mathfrak{v}=Z(\mathfrak{n})^{\perp}$. If $\mathfrak{n}$ is abelian and $\mathfrak{n} \neq\{0\}$, then there are two ordered decompositions of $\mathfrak{n}$, namely $(\mathfrak{z}, \mathfrak{v})=$ $(Z(\mathfrak{n}),\{0\})=(\mathfrak{n},\{0\})$ and $(\mathfrak{z}, \mathfrak{v})=(\{0\}, \mathfrak{n})$.

Proof Suppose that $(\mathfrak{z}, \mathfrak{v})$ is an ordered decomposition of $\mathfrak{n}$. Then $\mathfrak{v}$ is uniquely determined by $\mathfrak{z}$, namely $\mathfrak{v}=\mathfrak{z}^{\perp}$. Since $[\mathfrak{z}, \mathfrak{z}]=\{0\}$, we know that $\mathfrak{z}$ is a subvector space of $Z(\mathfrak{n})$ (even a subalgebra). If $\mathfrak{z}=\{0\}$, then $[\mathfrak{n}, \mathfrak{n}] \subseteq \mathfrak{z}=\{0\}$. In this case, $\mathfrak{n}$ is abelian and $(\mathfrak{z}, \mathfrak{v})=(\{0\}, \mathfrak{n})$.

Suppose now that $\mathfrak{z} \neq\{0\}$. We have to prove that $\mathfrak{z}=Z(\mathfrak{n})$. For contradiction assume that $\mathfrak{z} \neq Z(\mathfrak{n})$, hence $\operatorname{dim} \mathfrak{z}<\operatorname{dim} Z(\mathfrak{n})$. Then there is a non-trivial element $X \in \mathfrak{v} \cap Z(\mathfrak{n})$. Fix some $Z \in \mathfrak{z}, Z \neq 0$. For all $Y \in \mathfrak{v}$ it follows that

$$
\langle J(Z) X, Y\rangle=\langle Z,[X, Y]\rangle=0 .
$$

Thus $J(Z) X=0$. But then

$$
|J(Z) X|=0 \neq|Z| \cdot|X|,
$$

which is a contradiction to $(\mathrm{H} 2)$. Therefore, $\mathfrak{z}=Z(\mathfrak{n})$.

This shows that for non-abelian $\mathfrak{n}$ or for $\mathfrak{n}=\{0\}$, the pair $(\mathfrak{z}, \mathfrak{v})=\left(Z(\mathfrak{n}), Z(\mathfrak{n})^{\perp}\right)$ is the only candidate for an ordered decomposition of $\mathfrak{n}$. Because there is at least one by hypothesis, $\left(Z(\mathfrak{n}), Z(\mathfrak{n})^{\perp}\right)$ is indeed an ordered decomposition of $\mathfrak{n}$. For non-trivial abelian $\mathfrak{n}$ we have the two candidates $(\{0\}, \mathfrak{n})$ and $(\mathfrak{n},\{0\})$, which both clearly satisfy (H1) and (H2).

Let $\mathfrak{n}$ be a non-trivial abelian $H$-type algebra. Then $\mathfrak{n}$ admits the two ordered decompositions $(\mathfrak{n},\{0\})$ and $(\{0\}, \mathfrak{n})$. The isomorphism $\mathrm{id}_{\mathfrak{n}}$ of $\mathfrak{n}$ as a Euclidean Lie algebra does not respect these decompositions. In Sect. 2.3 we will see that preserving the decompositions is essential for the bijection between $H$-type algebras and $C$-module structures. Therefore, from now on, we will always consider an $H$-type algebra $\mathfrak{n}$ as being equipped with a (fixed) ordered decomposition and denote it by $\mathfrak{n}=(\mathfrak{z}, \mathfrak{v}, J)$ or, briefly, by $(\mathfrak{z}, \mathfrak{v}, J)$. Although the map $J$ is determined by $\mathfrak{z}$ and $\mathfrak{v}$, we keep it in the triple to fix a notation for it.

Definition 2.3 Let $\mathfrak{n}_{j}=\left(\mathfrak{z}_{j}, \mathfrak{v}_{j}, J_{j}\right), j=1,2$, be $H$-type algebras. An isomorphism from $\mathfrak{n}_{1}$ to $\mathfrak{n}_{2}$ is a pair $(\varphi, \psi)$ of isomorphisms of Euclidean vector spaces $\varphi: \mathfrak{z}_{1} \rightarrow \mathfrak{z}_{2}$ and $\psi: \mathfrak{v}_{1} \rightarrow \mathfrak{v}_{2}$ such that the diagram

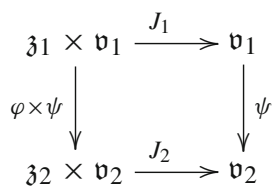

commutes.

The following lemma shows that isomorphism of $H$-type algebras is a refined notion of isomorphism of Euclidean Lie algebras.

Lemma 2.4 Let $\mathfrak{n}_{j}=\left(\mathfrak{z}_{j}, \mathfrak{v}_{j}, J_{j}\right), j=1,2$, be H-type algebras and suppose that the map $(\varphi, \psi): \mathfrak{n}_{1} \rightarrow \mathfrak{n}_{2}$ is an isomorphism. Then $\varphi \times \psi: \mathfrak{z}_{1} \oplus \mathfrak{v}_{1} \rightarrow \mathfrak{z}_{2} \oplus \mathfrak{v}_{2}$ is an isomorphism of Euclidean Lie algebras. 
Proof For all $Z \in \mathfrak{z}_{2}$ and all $X, Y \in \mathfrak{v}_{1}$ we have

$$
\begin{aligned}
\langle Z,[\psi(X), \psi(Y)]\rangle & =\left\langle J_{2}(Z)(\psi(X)), \psi(Y)\right\rangle=\left\langle\psi\left(J_{1}\left(\varphi^{-1}(Z)\right) X\right), \psi(Y)\right\rangle \\
& =\left\langle J_{1}\left(\varphi^{-1}(Z)\right) X, Y\right\rangle=\left\langle\varphi^{-1}(Z),[X, Y]\right\rangle \\
& =\langle Z, \varphi([X, Y])\rangle .
\end{aligned}
$$

Since $\left.\langle\cdot, \cdot\rangle\right|_{\mathfrak{z}_{2} \times \mathfrak{z}_{2}}$ is non-degenerate, it follows that $\varphi([X, Y])=[\psi(X), \psi(Y)]$. Define $\chi:=\varphi \times \psi$ and let $Z_{1}, Z_{2} \in \mathfrak{z} 1, X_{1}, X_{2} \in \mathfrak{v}_{1}$. Then

$$
\left[Z_{1}+X_{1}, Z_{2}+X_{2}\right]=\left[X_{1}, X_{2}\right]
$$

and

$$
\begin{aligned}
{\left[\chi\left(Z_{1}+X_{1}\right), \chi\left(Z_{2}+X_{2}\right)\right] } & =\left[\varphi\left(Z_{1}\right)+\psi\left(X_{1}\right), \varphi\left(Z_{2}\right)+\psi\left(X_{2}\right)\right] \\
& =\left[\psi\left(X_{1}\right), \psi\left(X_{2}\right)\right]=\varphi\left(\left[X_{1}, X_{2}\right]\right) \\
& =\chi\left(\left[Z_{1}+X_{1}, Z_{2}+X_{2}\right]\right) .
\end{aligned}
$$

Finally, $\chi$ is clearly an isomorphism of Euclidean vector spaces. This completes the proof.

Remark 2.5 Suppose that $\mathfrak{n}_{1}, \mathfrak{n}_{2}$ are non-degenerate $H$-type algebras and let the map $\chi: \mathfrak{n}_{1}$ $\rightarrow \mathfrak{n}_{2}$ be an isomorphism between $\mathfrak{n}_{1}$ and $\mathfrak{n}_{2}$ as Euclidean Lie algebras. Then $\chi\left(Z\left(\mathfrak{n}_{1}\right)\right)=$ $Z\left(\mathfrak{n}_{2}\right)$. Hence Lemma 2.2 implies that $\chi$ is an isomorphism of $H$-type algebras. In turn, Lemma 2.4 shows that the isomorphisms between $\mathfrak{n}_{1}$ and $\mathfrak{n}_{2}$ as $H$-type algebras coincide with the isomorphisms between $\mathfrak{n}_{1}$ and $\mathfrak{n}_{2}$ as Euclidean Lie algebras.

For an $H$-type algebra $(\mathfrak{z}, \mathfrak{v}, J)$ we often write $J_{Z} X$ instead of $J(Z) X$, and we abbreviate the set $\left\{J_{Z} \mid Z \in \mathfrak{z}\right\}$ with $J_{\mathfrak{z}}$.

Definition 2.6 An $H$-type algebra $\mathfrak{n}=(\mathfrak{z}, \mathfrak{v}, J)$ is said to satisfy the $J^{2}$-condition if

$$
\forall X \in \mathfrak{v} \forall Z_{1}, Z_{2} \in \mathfrak{z}:\left(\left\langle Z_{1}, Z_{2}\right\rangle=0 \Rightarrow \exists Z_{3} \in \mathfrak{z}: J_{Z_{1}} J_{Z_{2}} X=J_{Z_{3}} X\right) .
$$

If $\mathfrak{n}$ is abelian, then (H3) is trivially satisfied.

$2.2 C$-module structures and the $J^{2}$-condition

A $C$-module structure is a triple $(C, V, J)$ consisting of two Euclidean vector spaces $C$ and $V$ and an $\mathbb{R}$-bilinear map $J: C \times V \rightarrow V$ satisfying the following properties:

(M1) there exists $e \in C \backslash\{0\}$ such that $J(e, v)=v$ for all $v \in V$,

(M2) for all $\zeta \in C$ and all $v \in V$ we have $|J(\zeta, v)|=|\zeta||v|$.

The cases where $V=\{0\}$ or $C=\mathbb{R} e$ are not excluded. We refer to these as degenerate. If $V \neq\{0\}$ and $C \neq \mathbb{R} e$, then the $C$-module structure $(C, V, J)$ is called non-degenerate. For brevity, a $C$-module structure $(C, V, J)$ is sometimes called a $C$-module structure ${ }^{1}$ on $V$. One easily proves the following lemma.

Lemma 2.7 Let $(C, V, J)$ be a $C$-module structure. If $V \neq\{0\}$, then the element e in $(M 1)$ is uniquely determined and of unit length.

1 The " $C$ " is " $C$-module structure" or in " $C$-module structure on $V$ " does not refer to the Euclidean space $C$ in the triple $(C, V, J)$. Hence, if $\left(C^{\prime}, V, J^{\prime}\right)$ satisfies (M1) and (M2), then it is still called a $C$-module structure on $V$. 
The construction of a symmetric space from a $C$-module structure (satisfying the $J^{2}$-condition defined below) depends on the choice of $e$ in (M1) and uses $|e|=1$. If $(C, V, J)$ is a $C$-module structure with $V=\{0\}$, then $J$ vanishes everywhere. Thus every element $a \in C \backslash\{0\}$ satisfies $J(a, \cdot)=\mathrm{id}_{V}$. In this case, we endow $C$ with a distinguished vector $e$ of unit length and fix it (sometimes) in the notation as $(C, e, V, J)$. The influence of the particular choice of $e$ on the constructed symmetric space is much weaker than that of the different ordered decompositions of degenerate $H$-type algebras. In fact, the choice of $e$ determines the orthogonal decomposition $C=\mathbb{R} e \oplus C^{\prime}$ (see below). If $e_{1}, e_{2}$ are two choices for $e$, then there is an isomorphism between $\mathbb{R} e_{1} \oplus C_{1}^{\prime}$ and $\mathbb{R} e_{2} \oplus C_{2}^{\prime}$ as Euclidean vector spaces which respects the decompositions. In turn, $\left(C, e_{1}, V, J\right)$ and $\left(C, e_{2}, V, J\right)$ are isomorphic as $C$-module structures (see below for the definition of isomorphism).

If $(C, V, J)$ is a non-degenerate $C$-module structure, then the element $e$ in (M1) is unique by Lemma 2.7. For reasons of uniformity, also in this case, we will often use the notation $(C, e, V, J)$ for $(C, V, J)$.

If $(C, V, J)$ is a $C$-module structure, then we will use $J_{\zeta} v$ or $\zeta v$ to abbreviate $J(\zeta, v)$. Further, we set $C v:=\{\zeta v \mid \zeta \in C\}$ for $v \in V$.

A $C$-module structure $(C, V, J)$ is said to satisfy the $J^{2}$-condition if

$$
C(C v)=C v \text { for all } v \in V .
$$

In this case, $V$ is called ${ }^{2}$ a $J^{2} C$-module and $(C, V, J)$ a $J^{2} C$-module structure.

Let $\left(C_{1}, e_{1}, V_{1}, J_{1}\right)$ and $\left(C_{2}, e_{2}, V_{2}, J_{2}\right)$ be $C$-module structures. An isomorphism ${ }^{3}$ from $\left(C_{1}, e_{1}, V_{1}, J_{1}\right)$ to $\left(C_{2}, e_{2}, V_{2}, J_{2}\right)$ is a pair $(\varphi, \psi)$ of isomorphisms of Euclidean vector spaces $\psi: V_{1} \rightarrow V_{2}$ and $\varphi: C_{1} \rightarrow C_{2}$ with $\varphi\left(e_{1}\right)=e_{2}$ such that the diagram

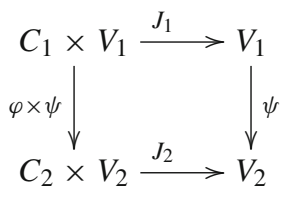

commutes.

The requirement that $\varphi\left(e_{1}\right)=e_{2}$ is relevant only if one of the $C$-module structures is degenerate. In fact, if $\left(C_{1}, e_{1}, V_{1}, J_{1}\right)$ and $\left(C_{2}, e_{2}, V_{2}, J_{2}\right)$ are non-degenerate $C$-module structures and $(\varphi, \psi)$ is a pair of isomorphisms of Euclidean vector spaces $\psi: V_{1} \rightarrow V_{2}$ and $\varphi: C_{1} \rightarrow C_{2}$ such that $J_{2} \circ(\varphi \times \psi)=\psi \circ J_{1}$, then

$$
J_{2}\left(\varphi\left(e_{1}\right), v\right)=\psi\left(J_{1}\left(e_{1}, \psi^{-1}(v)\right)\right)=v
$$

for each $v \in V$. The uniqueness of $e_{2}$ shows that $\varphi\left(e_{1}\right)=e_{2}$.

For a $C$-module structure $(C, e, V, J)$ let $C^{\prime}:=e^{\perp}$ denote the orthogonal complement of $\mathbb{R} e$ in $C$. For $\zeta=a e+z \in C$ with $a \in \mathbb{R}$ and $z \in C^{\prime}$ we set $\operatorname{Re} \zeta:=a$, the real part of $\zeta$, and $\operatorname{Im} \zeta:=z$, the imaginary part ${ }^{4}$ of $\zeta$. Further we set $\bar{\zeta}:=a e-z$, the conjugate of $\zeta$. We will use the identification $\mathbb{R} e \rightarrow \mathbb{R}, a e \mapsto a$, of Euclidean vector spaces.

2 As with the " $C$ " in " $C$-module structure", the " $J{ }^{2}$ " in " $J{ }^{2}$-condition" and " $J{ }^{2} C$-module" does not refer to the map $J$.

3 In [15] and [16] the condition $\varphi\left(e_{1}\right)=e_{2}$ is omitted from the definition. However, as discussed, this condition is needed and indeed this stronger notion of isomorphism is used in their work.

4 Note that, in contrast to the usual definition in complex analysis, if $C=\mathbb{C}$ and $\zeta=a+i b \in \mathbb{C}$, then one has here $\operatorname{Im} \zeta=i b$. 
2.3 Bijection between $H$-type algebras and $C$-module structures

Let $\mathfrak{n}=(\mathfrak{z}, \mathfrak{v}, J)$ be an $H$-type algebra. Endow $\mathbb{R}$ with the standard inner product and consider the Euclidean direct sum $\mathfrak{c}:=\mathbb{R} \oplus \mathfrak{z}$. The map $\widetilde{J}: \mathfrak{c} \times \mathfrak{v} \rightarrow \mathfrak{v}$, defined by

$$
\widetilde{J}(t+Z, X):=t X+J_{Z} X
$$

for all $t+Z \in \mathbb{R} \oplus \mathfrak{z}, X \in \mathfrak{v}$, is $\mathbb{R}$-bilinear. Since $\left\langle J_{Z} X, X\right\rangle=\langle Z,[X, X]\rangle=0$, we further find

$$
\begin{aligned}
|\widetilde{J}(t+Z, X)|^{2} & =\left|t X+J_{Z} X\right|^{2}=t^{2}|X|^{2}+\left|J_{Z} X\right|^{2} \\
& =t^{2}|X|^{2}+|Z|^{2}|X|^{2}=\left(t^{2}+|Z|^{2}\right)|X|^{2} \\
& =|t+Z|^{2}|X|^{2},
\end{aligned}
$$

hence $|\widetilde{J}(t+Z, X)|=|t+Z||X|$. Moreover, for each $X \in \mathfrak{v}$ we have

$$
\widetilde{J}(1, X)=X .
$$

Therefore, $(\mathfrak{c}, \mathfrak{v}, \widetilde{J})$ is a $C$-module structure with $e=1$. The condition (H3) is easily seen to be equivalent to

$$
\widetilde{J}(\mathfrak{c}) \widetilde{J}(\mathfrak{c}) X=\widetilde{J}(\mathfrak{c}) X \text { for all } X \in \mathfrak{v} .
$$

Thus, $(\mathfrak{c}, \mathfrak{v}, \widetilde{J})$ satisfies the $J^{2}$-condition if and only if $\mathfrak{n}$ does. This construction provides an assignment of a $C$-module structure to each $H$-type algebra (with fixed ordered decomposition).

Vice versa, let $(C, e, V, J)$ be a $C$-module structure and let $[\cdot, \cdot]: V \times V \rightarrow C^{\prime}$ be the map defined by

$$
\langle z,[x, y]\rangle=\langle J(z, x), y\rangle
$$

for all $z \in C^{\prime}$, all $x, y \in V$. Riesz' Representation Theorem (or its finite-dimensional counterpart) shows that $[\cdot, \cdot]$ is well-defined. We extend $[\cdot, \cdot]$ to the Euclidean $\operatorname{direct} \operatorname{sum} C^{\prime} \oplus V$ by

$$
\left[z_{1}+v_{1}, z_{2}+v_{2}\right]:=\left[v_{1}, v_{2}\right]
$$

for all $z_{j}+v_{j} \in C^{\prime} \oplus V$. This map is $\mathbb{R}$-bilinear. Since $J_{z}$ is skew-symmetric for each $z \in C^{\prime}$ (cf. Sect. 2.5), the (extended) map $[\cdot, \cdot]$ is anti-symmetric. Moreover, $[V, V] \subseteq C^{\prime}$ and $\left[V, C^{\prime}\right]=\left[C^{\prime}, C^{\prime}\right]=\{0\}$ imply the Jacobi identity for $[\cdot, \cdot]$. Thus, $C^{\prime} \oplus V$ endowed with $[\cdot, \cdot]$ is a Euclidean Lie algebra. Let $J^{\prime}: C^{\prime} \rightarrow \operatorname{End}_{\mathrm{vs}}(V)$ denote the map $J^{\prime}(z)(v)=J(z, v)$. Then $\left(C^{\prime}, V, J^{\prime}\right)$ is an $H$-type algebra. Using the equivalence of (H3) and (H3'), we see that this $H$-type algebra satisfies the $J^{2}$-condition if and only if $(C, V, J)$ does so.

Using the identification $e=1$ from Sect. 2.2, the construction of a $C$-module structure from an $H$-type algebra

$$
(\mathfrak{z}, \mathfrak{v}, J) \mapsto(\mathbb{R} \oplus \mathfrak{z}, 1, \mathfrak{v}, \widetilde{J})
$$

and that of an $H$-type algebra from a $C$-module structure

$$
(C, e, V, J) \mapsto\left(C^{\prime}, V, J^{\prime}\right)
$$

are inverse to each other. Moreover, one easily sees that these constructions are equivariant under isomorphisms of $C$-module structures, respectively of $H$-type algebras. More precisely, if $(\varphi, \psi):\left(\mathfrak{z}_{1}, \mathfrak{v}_{1}, J_{1}\right) \rightarrow\left(\mathfrak{z}_{2}, \mathfrak{v}_{2}, J_{2}\right)$ is an isomorphism of $H$-type algebras, then 


$$
(\text { id } \times \varphi, \psi):\left(\mathbb{R} \oplus \mathfrak{z}_{1}, 1, \mathfrak{v}_{1}, \widetilde{J}_{1}\right) \rightarrow\left(\mathbb{R} \oplus \mathfrak{z}_{2}, 1, \mathfrak{v}_{2}, \widetilde{J}_{2}\right)
$$

is an isomorphism of $C$-module structures.

Conversely, if $(\varphi, \psi):\left(C_{1}, e_{1}, V_{1}, J_{1}\right) \rightarrow\left(C_{2}, e_{2}, V_{2}, J_{2}\right)$ is an isomorphism of $C$-module structures, then $\varphi\left(C_{1}^{\prime}\right)=C_{2}^{\prime}$ and the map

$$
\left(\left.\varphi\right|_{C_{1}^{\prime}}, \psi\right):\left(C_{1}^{\prime}, V_{1}, J_{1}^{\prime}\right) \rightarrow\left(C_{2}^{\prime}, V_{2}, J_{2}^{\prime}\right)
$$

is an isomorphism of $H$-type algebras.

\subsection{The model $D$}

Let $\mathfrak{n}=(\mathfrak{z}, \mathfrak{v}, J)$ be an $H$-type algebra. Further let $\mathfrak{a}$ be a one-dimensional Euclidean Lie algebra and fix an element $H$ of unit length in $\mathfrak{a}$. Then $\mathfrak{a}$ is spanned by $H$. We denote by $\mathfrak{s}$ the Euclidean direct sum Lie algebra $\mathfrak{a} \oplus \mathfrak{n}=\mathfrak{a} \oplus \mathfrak{z} \oplus \mathfrak{v}$ endowed with the Lie bracket that is determined by requiring that

$$
\begin{array}{ll}
{[H, X]=\frac{1}{2} X} & \text { for all } X \in \mathfrak{v} \\
{[H, Z]=Z} & \text { for all } Z \in \mathfrak{z}
\end{array}
$$

and that equals the original Lie bracket on $\mathfrak{n}$, when restricted to $\mathfrak{n}$.

Let $\exp (\mathfrak{s})$ be the connected, simply connected Lie group with Lie algebra $\mathfrak{s}$. We identify the tangent $\operatorname{space}$ to $\exp (\mathfrak{s})$ at the identity with $\mathfrak{s}$. Further we endow $\exp (\mathfrak{s})$ with the left$\exp (\mathfrak{s})$-invariant Riemannian metric that coincides with the inner product on $\mathfrak{s}$ at the identity of $\exp (\mathfrak{s})$. We parametrize $\exp (\mathfrak{s})$ by

$$
\left\{\begin{aligned}
\mathbb{R}^{+} \times \mathfrak{z} \times \mathfrak{v} & \rightarrow \exp (\mathfrak{s}) \\
(t, Z, X) & \mapsto \exp (Z+X) \exp ((\log t) H)
\end{aligned}\right.
$$

and set $S:=\mathbb{R}^{+} \times \mathfrak{z} \times \mathfrak{v}$. By requiring this parametrization to be a diffeomorphism and an isometry, $S$ inherits the structure of a connected, simply connected Lie group with Riemannian metric. The Campbell-Baker-Hausdorff formula for $\mathfrak{n}=\mathfrak{z} \oplus \mathfrak{v}$ shows that the group operations on $\exp (\mathfrak{s})$ correspond on $S$ to the group operations

$$
\begin{aligned}
\left(t_{1}, Z_{1}, X_{1}\right)\left(t_{2}, Z_{2}, X_{2}\right) & =\left(t_{1} t_{2}, Z_{1}+t_{1} Z_{2}+\frac{1}{2} t_{1}^{1 / 2}\left[X_{1}, X_{2}\right], X_{1}+t_{1}^{1 / 2} X_{2}\right) \\
(t, Z, X)^{-1} & =\left(t^{-1},-t^{-1} Z,-t^{-1 / 2} X\right)
\end{aligned}
$$

for all $\left(t_{j}, Z_{j}, X_{j}\right),(t, Z, X) \in S$. The differential structure on $S$ coincides with the differential structure on the open subset $\mathbb{R}^{+} \times \mathfrak{z} \times \mathfrak{v}$ of some $\mathbb{R}^{m}$. Now let

$$
D:=\left\{(t, Z, X) \in \mathbb{R} \times \mathfrak{z} \times\left.\mathfrak{v}\left|t>\frac{1}{4}\right| X\right|^{2}\right\}
$$

and consider the bijection

$$
\Theta:\left\{\begin{array}{l}
\mathbb{R} \times \mathfrak{z} \times \mathfrak{v} \rightarrow \mathbb{R} \times \mathfrak{z} \times \mathfrak{v} \\
(t, Z, X) \mapsto\left(t+\frac{1}{4}|X|^{2}, Z, X\right) .
\end{array}\right.
$$

Then $\Theta(S)=D$, so that we define the structure of a Riemannian manifold on $D$ by requiring $\Theta$ to be an isometry. The differential structure on $D$ is identical to that of $D$ being an open 
subset of $\mathbb{R} \times \mathfrak{z} \times \mathfrak{v}$. Moreover, $\Theta$ induces a simply transitive action of $S$ on $D$ by defining

$$
s \cdot p:=\Theta\left(s \Theta^{-1}(p)\right)
$$

for $s \in S$ and $p \in D$. In coordinates $s=\left(t_{s}, Z_{s}, X_{s}\right)$ and $p=\left(t_{p}, Z_{p}, X_{p}\right)$ this action reads

$$
s \cdot p=\left(t_{s} t_{p}+\frac{1}{4}\left|X_{s}\right|^{2}+\frac{1}{2} t_{s}^{1 / 2}\left\langle X_{s}, X_{p}\right\rangle, Z_{s}+t_{s} Z_{p}+\frac{1}{2} t_{s}^{1 / 2}\left[X_{s}, X_{p}\right], X_{s}+t_{s}^{1 / 2} X_{p}\right)
$$

Due to the definition of the Riemannian metric, $S$ obviously acts by isometries. We call $o_{D}:=(1,0,0)$ the base point of $D$. The geodesic inversion $\sigma$ of $D$ at $o_{D}$ is given by

$$
\sigma(t, Z, X)=\frac{1}{t^{2}+|Z|^{2}}\left(t,-Z,\left(-t+J_{Z}\right) X\right)
$$

for all $(t, Z, X) \in D$. Then $\sigma$ is an isometry, and hence $D$ a symmetric space, if and only if $\mathfrak{n}$ satisfies the $J^{2}$-condition. In this case, $D$ has rank one and, if in addition $\mathfrak{n}$ is non-trivial, then $D$ is of noncompact type. If $\mathfrak{n}=\{0\}$, then the constructed space $D$ is the rank one Euclidean symmetric space $\mathbb{R}$.

Conversely, let $D$ be a rank one symmetric space of noncompact type. Suppose that $\mathfrak{g}$ is the simple Lie algebra of the Lie group of Riemannian isometries of $D$. Let $\vartheta$ be a Cartan involution of $\mathfrak{g}$, and let $\mathfrak{k}$ and $\mathfrak{p}$ be its +1 - respectively -1 -eigenspace. Fix a maximal abelian subalgebra $\mathfrak{a}$ of $\mathfrak{p}$ and choose a vector $H \in \mathfrak{a}$ which spans $\mathfrak{a}$. Then the decomposition of $\mathfrak{g}$ into restricted root spaces is

$$
\mathfrak{g}=\mathfrak{g}_{-2 \alpha} \oplus \mathfrak{g}_{-\alpha} \oplus(\mathfrak{a} \oplus \mathfrak{m}) \oplus \mathfrak{g}_{\alpha} \oplus \mathfrak{g}_{2 \alpha},
$$

where

$$
\mathfrak{g}_{\beta}:=\{X \in \mathfrak{g} \mid[H, X]=\beta(H) X\}
$$

for the linear functional $\beta: \mathfrak{a} \rightarrow \mathbb{R}$ and

$$
\mathfrak{m}:=\{X \in \mathfrak{k} \mid[H, X]=0\} .
$$

We suppose that $H$ is normalized such that $\alpha(H)=\frac{1}{2}$. If we set $p:=\operatorname{dim} \mathfrak{g}_{\alpha}$ and $q:=\operatorname{dim} \mathfrak{g}_{2 \alpha}$ and if we endow $\mathfrak{n}:=\mathfrak{g}_{2 \alpha} \oplus \mathfrak{g}_{\alpha}$ with the inner product

$$
\langle X, Y\rangle:=-\frac{1}{p+4 q} B(X, \vartheta Y)
$$

where $B$ is the Killing form of $\mathfrak{g}$, then $\mathfrak{n}=\left(\mathfrak{g}_{2 \alpha}, \mathfrak{g}_{\alpha}, J\right)$ is an $H$-type algebra with $J^{2}$ condition. The symmetric space constructed from $\left(\mathfrak{g}_{2 \alpha}, \mathfrak{g}_{\alpha}, J\right)$ is exactly $D$. This means that each rank one Riemannian symmetric space of noncompact type arises from the construction above.

\subsection{The ball model $B$}

Let $(C, e, V, J)$ be a $J^{2} C$-module structure and let $W:=C \oplus V$ be the Euclidean direct sum of $C$ and $V$. Consider the unit disc

$$
B:=\{w \in W|| w \mid<1\}
$$

in $W$ and endow it with the differential structure induced from $W$. In the following we will define a Riemannian metric on $B$ with respect to which $B$ is a rank one Riemannian symmetric space of noncompact type if $(C, V) \neq(\mathbb{R} e,\{0\})$. 
Polarization of the equation in (M2) shows that we have

$$
\langle\zeta u, \eta v\rangle+\langle\eta u, \zeta v\rangle=2\langle\zeta, \eta\rangle\langle u, v\rangle
$$

for all $\zeta, \eta \in C$ and all $u, v \in V$. For $\zeta \in C$ let $J_{\zeta}^{*}$ denote the adjoint of $J_{\zeta}$. Then (2.6) implies

$$
J_{\zeta}^{*}=J_{\bar{\zeta}}
$$

Moreover we have

$$
J_{\bar{\zeta}} J_{\zeta}=|\zeta|^{2} \mathrm{id}_{V}=J_{\zeta} J_{\bar{\zeta}}
$$

Thus, if we set $\zeta^{-1}:=|\zeta|^{-2} \bar{\zeta}$ for $\zeta \in C \backslash\{0\}$, then we have

$$
\zeta^{-1}(\zeta v)=v=\zeta\left(\zeta^{-1} v\right)
$$

for all $v \in V$. In Sect. 4.1 we will see that, if $V \neq\{0\}$, there is a multiplication on $C$ such that $\zeta^{-1}$ is the inverse of $\zeta$.

Definition 2.8 Let $(\zeta, v),(\eta, u) \in W \backslash\{0\}$. Then $(\zeta, v)$ is called equivalent to $(\eta, u)$, if either $\zeta=0=\eta$ and $u \in C v$, or $\zeta \neq 0 \neq \eta$ and $\zeta^{-1} v=\eta^{-1} u$. In this case, we write $(\zeta, v) \sim(\eta, u)$

One easily proves that $\sim$ is an equivalence relation on $W \backslash\{0\}$. For an element $w \in$ $W \backslash\{0\}$ let

$$
C w:=\left\{w^{\prime} \in W \backslash\{0\} \mid w^{\prime} \sim w\right\} \cup\{0\}
$$

denote the equivalence class of $w$ together with the element $0 \in W$. Since $B$ is an open subset of the real vector space $W$, we shall identify the tangent space $T_{w} B$ to the point $w \in B$ with $W$. The Riemannian metric $w \mapsto\langle\cdot, \cdot\rangle_{w-}$ on $B$ is defined by

$$
\langle X, Y\rangle_{0-}:=4\langle X, Y\rangle \text { on } T_{0} B
$$

and, for $w \in B \backslash\{0\}$, by

$$
\langle X, Y\rangle_{w-}:= \begin{cases}4 \frac{\langle X, Y\rangle}{\left(1-|w|^{2}\right)^{2}} & \text { if } X, Y \in C w \\ 4 \frac{\langle X, Y\rangle}{1-|w|^{2}} & \text { if } X, Y \in(C w)^{\perp} \\ 0 & \text { if } X \in C w, Y \in(C w)^{\perp} \text { (or vice versa). }\end{cases}
$$

2.6 The Cayley transform

Let $\mathfrak{n}=(\mathfrak{z}, \mathfrak{v}, J)$ be a non-trivial $H$-type algebra which satisfies the $J^{2}$-condition. Further let $\mathfrak{a}$ be a one-dimensional Euclidean Lie algebra with fixed unit length vector $H$. Suppose that $(C, e, V, J)$ is the $J^{2} C$-module structure associated to $(\mathfrak{z}, \mathfrak{v}, J)$ (see Sect. 2.3). We consider the Riemannian symmetric spaces $D$ and $B$ which are constructed in Sect. 2.4, respectively, 2.5.

The Cayley transform (in $\mathbb{R} \times \mathfrak{z} \times \mathfrak{v}$-coordinates, see [7, (2.10a)])

$$
\mathcal{C}:\left\{\begin{array}{l}
B \rightarrow D \\
(t, Z, X) \mapsto \frac{1}{(1-t)^{2}+|Z|^{2}}\left(1-t^{2}-|Z|^{2}, 2 Z, 2\left(1-t+J_{Z}\right) X\right)
\end{array}\right.
$$


is clearly a diffeomorphism from $B$ to $D$. Its inverse (see [7, (2.10b)]) is given by

$$
\mathcal{C}^{-1}:\left\{\begin{array}{l}
D \rightarrow B \\
(t, Z, X) \mapsto \frac{1}{(1+t)^{2}+|Z|^{2}}\left(-1+t^{2}+|Z|^{2}, 2 Z,\left(1+t-J_{Z}\right) X\right) .
\end{array}\right.
$$

Proposition 2.9 The Cayley transform is an isometry.

Proof In [7], the pullback of the Riemannian metric of $D$ to $B$ via $\mathcal{C}$ is described as follows: Let $p \in B$. Denote the tangent space to $B$ at $p$ by $T_{p} B$, which we identify with $\mathbb{R} \times \mathfrak{z} \times \mathfrak{v}$. If $\|\mathfrak{X}\|_{p}$ denotes the norm of $\mathfrak{X} \in T_{p} B$ induced by the pullback of the Riemannian metric on $D$, then

$$
\|\mathfrak{X}\|_{0}=2|\mathfrak{X}|
$$

for all $\mathfrak{X} \in T_{0} B$. For $p \in B \backslash\{0\}$ we have

$$
\|\mathfrak{X}\|_{p}^{2}= \begin{cases}4 \frac{|\mathfrak{X}|^{2}}{1-|p|^{2}} & \text { if } \mathfrak{X} \in T_{p}^{(1)} \\ 4 \frac{|\mathfrak{X}|^{2}}{\left(1-|p|^{2}\right)^{2}} & \text { if } \mathfrak{X} \in \mathbb{R} p \oplus T_{p}^{(2)}\end{cases}
$$

where

$$
T_{p} B=\mathbb{R} p \oplus T_{p}^{(2)} \oplus T_{p}^{(1)}
$$

is a direct sum which is orthogonal w.r.t. Euclidean metric and Riemannian inner product on $T_{p} B$. Theorem 6.8 in [7] provides explicit formulas for $T_{p}^{(1)}$ and $\mathbb{R} p \oplus T_{p}^{(2)}$, which we will state in the following. For $X \in \mathfrak{v}$ let $\mathfrak{j}(X):=J_{\mathfrak{z}} X$ and define $\mathfrak{k}(X)$ to be the orthogonal complement of $\mathbb{R} X$ in

$$
\mathfrak{j}(X)^{\perp}=\left\{Y \in \mathfrak{v} \mid \forall Z \in \mathfrak{z}:\left\langle J_{Z} X, Y\right\rangle=0\right\} .
$$

For $p=(t, Z, X) \in B \backslash\{0\}$ we have

(i) $T_{p}^{(1)}=\mathfrak{v}$ and $\mathbb{R} p \oplus T_{p}^{(2)}=\mathbb{R} \oplus \mathfrak{z}$ if $X=0$,

(ii) $T_{p}^{(1)}=\mathbb{R} \oplus \mathfrak{z} \oplus \mathfrak{k}(X)$ and $\mathbb{R} p \oplus T_{p}^{(2)}=\mathfrak{j}(X) \oplus \mathbb{R} X$ if $(t, Z)=(0,0)$,

(iii) in the remaining cases,

$$
T_{p}^{(1)}=\mathfrak{k}(X) \oplus\left\{\left(|X|^{2} u,|X|^{2} W,-\left(u+J_{W}\right)\left(t-J_{Z}\right) W\right) \mid W \in \mathfrak{z}, u \in \mathbb{R}\right\}
$$

and

$$
\mathbb{R} p \oplus T_{p}^{(2)}=\left\{\left(\left(t^{2}+|Z|^{2}\right) u,\left(t^{2}+|Z|^{2}\right) W,\left(u+J_{W}\right)\left(t-J_{Z}\right) X\right) \mid W \in \mathfrak{z}, u \in \mathbb{R}\right\} .
$$

Note that this subsumes the degenerate cases. On $T_{0} B$, the Riemannian inner product (2.10) obviously coincides with this one. For $p \in B \backslash\{0\}$, (2.11) implies that it suffices to show that $\mathbb{R} p \oplus T_{p}^{(2)}=C p$. To that end let $p=(\zeta, v)=(t, Z, X) \in B \backslash\{0\}$ (hence $\zeta=(t, Z) \in$ $C=\mathbb{R} \times \mathfrak{z}$ and $v=X \in V=\mathfrak{v})$. If $v=0$, then

$$
C p=C \times\{0\}=C=\mathbb{R} \times \mathfrak{z}=\mathbb{R} p \oplus T_{p}^{(2)} .
$$

If $\zeta=0$, then

$$
C p=C v=\left(\mathbb{R}+J_{\mathfrak{z}}\right) X=\mathbb{R} p \oplus T_{p}^{(2)} .
$$

If $\zeta \neq 0$ and $v \neq 0$, then $(\eta, u) \in C p$ if and only if $\eta \neq 0$ and $\zeta^{-1} v=\eta^{-1} u$, or $(\eta, u)=0$. This means that in both cases

$$
u=J_{\eta} J_{\zeta^{-1}} v=|\zeta|^{-2} J_{\eta} J_{\bar{\zeta}} v=J_{|\zeta|^{-2} \eta} J_{\bar{\zeta}} v .
$$


Hence

$$
C p=\left\{\left(\eta, J_{|\zeta|^{-2} \eta} J_{\bar{\zeta}} v\right) \mid \eta \in C\right\}=\left\{\left(|\zeta|^{2} \xi, J_{\xi} J_{\bar{\zeta}} v\right) \mid \xi \in C\right\}=\mathbb{R} p \oplus T_{p}^{(2)} .
$$

This completes the proof.

Note that for $\mathfrak{n}=\{0\}$, respectively, $(C, V)=(\mathbb{R} e,\{0\})$ Proposition 2.9 shows that the model $B$ is isometric to the Euclidean symmetric space $\mathbb{R}$.

2.7 The map $\beta_{2}$

Let $(C, e, V, J)$ be a $J^{2} C$-module structure. In this section, we introduce a map

$$
\beta_{2}: V \times V \rightarrow C,
$$

which will be shown to be $C$-hermitian, i.e., $\beta_{2}$ is $\mathbb{R}$-bilinear and for all $u, v \in V$ we have $\beta_{2}(u, v)=\overline{\beta_{2}(v, u)}$. The map $\beta_{2}$ encodes the inner product and the Lie bracket on $V$. We define $\beta_{2}: V \times V \rightarrow C$ by $^{5}$

$$
\left\langle\beta_{2}(v, u), \zeta\right\rangle:=\left\langle J_{\zeta} u, v\right\rangle \text { for all } \zeta \in C .
$$

Lemma 2.10 For $\zeta, \eta \in C$ we have $\langle\eta, \bar{\zeta}\rangle=\langle\bar{\eta}, \zeta\rangle$.

Proof Let $\zeta=a+x$ and $\eta=b+y\left(a, b \in \mathbb{R}, x, y \in C^{\prime}\right)$ be the decompositions of $\zeta$ and $\eta$ w.r.t. $C=\mathbb{R} \oplus C^{\prime}$. Since $\mathbb{R}$ and $C^{\prime}$ are orthogonal, we find

$$
\begin{aligned}
\langle\bar{\zeta}, \eta\rangle & =\langle a, b\rangle-\langle x, b\rangle+\langle a, y\rangle-\langle x, y\rangle \\
& =\langle a, b\rangle-\langle x, y\rangle \\
& =\langle a, b\rangle+\langle x, b\rangle-\langle a, y\rangle-\langle x, y\rangle \\
& =\langle\zeta, \bar{\eta}\rangle .
\end{aligned}
$$

This proves the lemma.

Proposition 2.11 The map $\beta_{2}: V \times V \rightarrow C$ is $\mathbb{R}$-bilinear. Further we have

(i) $\beta_{2}(v, u)=\overline{\beta_{2}(u, v)}$ for all $u, v \in V$,

(ii) $\beta_{2}(v, v)=\langle v, v\rangle$ for all $v \in V$.

Proof One easily sees that $\beta_{2}$ is $\mathbb{R}$-bilinear. Using Lemma 2.10 we find

$$
\left\langle\beta_{2}(v, u), \zeta\right\rangle=\left\langle J_{\zeta} u, v\right\rangle=\left\langle u, J_{\bar{\zeta}} v\right\rangle=\left\langle\beta_{2}(u, v), \bar{\zeta}\right\rangle=\left\langle\overline{\beta_{2}(u, v)}, \zeta\right\rangle
$$

for all $u, v \in V$ and all $\zeta \in C$. Hence $\beta_{2}(v, u)=\overline{\beta_{2}(u, v)}$, which proves (i). Finally let $v \in V$. From $\beta_{2}(v, v)=\overline{\beta_{2}(v, v)}$ it follows that $\beta_{2}(v, v) \in \mathbb{R}$. Then

$$
\beta_{2}(v, v)=\left\langle\beta_{2}(v, v), e\right\rangle=\langle e v, v\rangle=\langle v, v\rangle,
$$

which shows (ii).

Lemma 2.12 For $v_{1}, v_{2} \in V$ we have

$$
\operatorname{Re} \beta_{2}\left(v_{1}, v_{2}\right)=\left\langle v_{1}, v_{2}\right\rangle \text { and } \operatorname{Im} \beta_{2}\left(v_{1}, v_{2}\right)=\left[v_{2}, v_{1}\right]
$$

5 This is $J^{*}$ in [16]. 
Proof Let $v_{1}, v_{2} \in V$. By Proposition 2.11 we have

$$
\begin{aligned}
\left|v_{1}\right|^{2}+2\left\langle v_{1}, v_{2}\right\rangle+\left|v_{2}\right|^{2} & =\left|v_{1}+v_{2}\right|^{2}=\beta_{2}\left(v_{1}+v_{2}, v_{1}+v_{2}\right) \\
& =\left|v_{1}\right|^{2}+\beta_{2}\left(v_{1}, v_{2}\right)+\beta_{2}\left(v_{2}, v_{1}\right)+\left|v_{2}\right|^{2} \\
& =\left|v_{1}\right|^{2}+\beta_{2}\left(v_{1}, v_{2}\right)+\overline{\beta_{2}\left(v_{1}, v_{2}\right)}+\left|v_{2}\right|^{2} \\
& =\left|v_{1}\right|^{2}+2 \operatorname{Re} \beta_{2}\left(v_{1}, v_{2}\right)+\left|v_{2}\right|^{2} .
\end{aligned}
$$

Hence $\operatorname{Re} \beta_{2}\left(v_{1}, v_{2}\right)=\left\langle v_{1}, v_{2}\right\rangle$. To show the second claim, note that Proposition $2.11 \mathrm{implies}$ that

$$
\operatorname{Im} \beta_{2}\left(v_{1}, v_{2}\right)=\frac{1}{2} \beta_{2}\left(v_{1}, v_{2}\right)-\frac{1}{2} \overline{\beta_{2}\left(v_{1}, v_{2}\right)}=\frac{1}{2} \beta_{2}\left(v_{1}, v_{2}\right)-\frac{1}{2} \beta_{2}\left(v_{2}, v_{1}\right) .
$$

For each $\zeta \in C^{\prime}$ it follows that

$$
\begin{aligned}
\left\langle\zeta, \operatorname{Im} \beta_{2}\left(v_{1}, v_{2}\right)\right\rangle & =\frac{1}{2}\left\langle\zeta, \beta_{2}\left(v_{1}, v_{2}\right)\right\rangle-\frac{1}{2}\left\langle\zeta, \beta_{2}\left(v_{2}, v_{1}\right)\right\rangle \\
& =\frac{1}{2}\left\langle J_{\zeta} v_{2}, v_{1}\right\rangle-\frac{1}{2}\left\langle J_{\zeta} v_{1}, v_{2}\right\rangle \\
& =\frac{1}{2}\left\langle J_{\zeta} v_{2}, v_{1}\right\rangle+\frac{1}{2}\left\langle v_{1}, J_{\zeta} v_{2}\right\rangle \\
& =\left\langle J_{\zeta} v_{2}, v_{1}\right\rangle=\left\langle\zeta,\left[v_{2}, v_{1}\right]\right\rangle .
\end{aligned}
$$

Since $\left.\langle\cdot, \cdot\rangle\right|_{C^{\prime} \times C^{\prime}}$ is non-degenerate and $\operatorname{Im} \beta_{2}\left(v_{1}, v_{2}\right) \in C^{\prime}$ and $\left[v_{2}, v_{1}\right] \in C^{\prime}$, it follows that $\operatorname{Im} \beta_{2}\left(v_{1}, v_{2}\right)=\left[v_{2}, v_{1}\right]$.

\subsection{The isometry group}

Let $\mathfrak{n}=(\mathfrak{z}, \mathfrak{v}, J)$ be a non-trivial $H$-type algebra which satisfies the $J^{2}$-condition, and let $\mathfrak{a}$ be a one-dimensional Euclidean Lie algebra. Construct the Euclidean Lie algebra $\mathfrak{s}$ and the spaces $S$ and $D$ as in Sect. 2.4. We denote by $(C, e, V, J)$ the $J^{2} C$-module structure $(\mathbb{R} \oplus \mathfrak{z}, 1, \mathfrak{v}, \widetilde{J})$ which is isomorphic to $\mathfrak{n}$. Let $G$ denote the full isometry group of $D$. Suppose that $N$, respectively, $A$ are the connected, simply connected Lie groups with Lie algebra $\mathfrak{n}$, respectively, a. Then $N$ and $A$ are subgroups of $S$, more precisely, $S$ is the semidirect product $A N$. In the parametrization of $S$, the groups $N$ and $A$ are given by

$$
N=\left\{n_{(Z, X)}:=(1, Z, X) \mid(Z, X) \in \mathfrak{z} \times \mathfrak{v}\right\}
$$

and

$$
A=\left\{a_{t}:=(t, 0,0) \mid t \in \mathbb{R}^{+}\right\} .
$$

Let $K$ be the stabilizer of the base point $o_{D}=(1,0,0)$ in $G$ and let $M:=Z_{K}(A)$ be the centralizer of $A$ in $K$. Recall the geodesic inversion

$$
\sigma(t, Z, X)=\frac{1}{t^{2}+|Z|^{2}}\left(t,-Z,\left(-t+J_{Z}\right) X\right)
$$

at the origin $o_{D}$ from Sect. 2.4.

Theorem 2.13 (Theorem 6.4 in [7]) The Lie group G has the Bruhat decomposition MAN $\cup$ $N \sigma M A N$. 
Let $\mathcal{X}:=\mathbb{R} \times \mathfrak{z} \times \mathfrak{v} \cup\{\infty\}$ be the one-point compactification of $\mathbb{R} \times \mathfrak{z} \times \mathfrak{v}$, where $\infty$ denotes the point at infinity. A compactification of $D$ is then given by the closure of $D$ in $\mathcal{X}$, namely

$$
\begin{aligned}
\bar{D}^{g} & =\left\{(t, Z, X) \in \mathbb{R} \times \mathfrak{z} \times\left.\mathfrak{v}\left|t \geq \frac{1}{4}\right| X\right|^{2}\right\} \cup\{\infty\} \\
& =\left\{(\zeta, v) \in C \times\left. V\left|\operatorname{Re} \zeta \geq \frac{1}{4}\right| v\right|^{2}\right\} \cup\{\infty\} .
\end{aligned}
$$

The space $\bar{D}^{g}$ is precisely the geodesic compactification of $D$, see, e.g., [3, Sect. I.2] or [8, Proposition 1.7.6]. Let $\bar{B}$ be the closed unit ball in $W=\mathbb{R} \times \mathfrak{z} \times \mathfrak{v}$. Then the Cayley transform $\mathcal{C}: B \rightarrow D$ extends (uniquely) to a homeomorphism $\bar{B} \rightarrow \bar{D}^{g}$. Therefore, [7, Corollary 6.2] amounts to the following proposition.

Proposition 2.14 The action of $G$ extends continuously to $\bar{D}^{g}$.

The Bruhat decomposition of $G$ implies that the stabilizer $G_{\infty}$ of $\infty$ in $G$ equals $M A N$.

For future purposes we need explicit formulas for the action of the groups $M, A$ and $N$ on $\bar{D}^{g}$. The action of $N$ and $A$ in $\mathbb{R} \times \mathfrak{z} \times \mathfrak{v}$-coordinates is already given in (2.5). Suppose that $(\zeta, v) \in \bar{D}^{g} \backslash\{\infty\}$. For $a_{t} \in A$ we have

$$
a_{t} \infty=\infty \quad \text { and } \quad a_{t}(\zeta, v)=\left(t \zeta, t^{1 / 2} v\right) .
$$

For $n_{(Z, X)} \in N$ we get $n_{(Z, X)} \infty=\infty$ and

$$
n_{(Z, X)}(\zeta, v)=\left(\frac{1}{4}|X|^{2}+Z+\zeta+\frac{1}{2} \beta_{2}(v, X), X+v\right) .
$$

In $C \times V$-coordinates, the geodesic inversion $\sigma$ reads as

$$
\sigma \infty=0, \quad \sigma 0=\infty
$$

and for $(\zeta, v) \in \bar{D}^{g} \backslash\{0, \infty\}$,

$$
\begin{aligned}
\sigma(\zeta, v) & =\frac{1}{(\operatorname{Re} \zeta)^{2}+|\operatorname{Im} \zeta|^{2}}\left(\operatorname{Re} \zeta,-\operatorname{Im} \zeta, J_{-\operatorname{Re} \zeta+\operatorname{Im} \zeta} v\right) \\
& =|\zeta|^{-2}\left(\bar{\zeta}, J_{-\bar{\zeta}} v\right)=\left(\zeta^{-1}, J_{-|\zeta|^{-2} \bar{\zeta}} v\right) \\
& =\left(\zeta^{-1},-\zeta^{-1} v\right)=\zeta^{-1}(1,-v)
\end{aligned}
$$

The Cayley transform in $C \times V$-coordinates is

$$
\mathcal{C}:\left\{\begin{array}{l}
B \rightarrow D \\
(\zeta, v) \mapsto|1-\zeta|^{-2}\left(1+2 \operatorname{Im} \zeta-|\zeta|^{2}, 2(1-\bar{\zeta}) v\right)
\end{array}\right.
$$

with

$$
\mathcal{C}^{-1}:\left\{\begin{array}{l}
D \rightarrow B \\
(\eta, u) \mapsto|1+\eta|^{-2}\left(-1+2 \operatorname{Im} \eta+|\eta|^{2}, 2(1+\bar{\eta}) u\right) .
\end{array}\right.
$$

The following proposition provides the explicit form of the group $M$.

Proposition 2.15 The group $M$ is the group of automorphisms of the $J^{2} C$-module structure $(C, e, V, J)$, that is, the elements of $M$ are the pairs $(\varphi, \psi)$ of orthogonal endomorphisms 
$\varphi: C \rightarrow C$ with $\varphi(e)=e$ and $\psi: V \rightarrow V$ such that the diagram

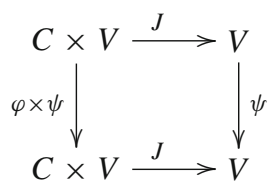

commutes. If $(\varphi, \psi) \in M$, then its action on $\bar{D}^{g}$ is given by $(\varphi, \psi)(\infty)=\infty$ and $(\varphi, \psi)(\zeta, v)=(\varphi(\zeta), \psi(v))$ for $(\zeta, v) \in \bar{D}^{g} \backslash\{\infty\}$.

Proof Let $\widetilde{G}:=\mathcal{C}^{-1} G \mathcal{C}$. For each $g \in G$ set $\widetilde{g}:=\mathcal{C}^{-1} g \mathcal{C}$, and for each subset $T$ of $G$ let $\widetilde{T}:=\mathcal{C}^{-1} T \mathcal{C}$ be the corresponding subset of $\widetilde{G}$. Clearly, $\widetilde{G}$ is the full isometry group of $B$. We will first characterize the centralizer $Z_{\widetilde{K}}(\widetilde{A})$ of $\widetilde{A}$ in $\widetilde{K}$ as a subgroup of $\widetilde{G}$. Let $\widetilde{M}$ denote the automorphism group of $(C, e, V, J)$ and define the action of $(\varphi, \psi) \in \widetilde{M}$ on $B$ by $(\varphi, \psi)(\zeta, v):=(\varphi(\zeta), \psi(v))$. We will show that $\widetilde{M}=Z_{\widetilde{K}}(\widetilde{A})$. By [15, Proposition 4.1] we have that $\widetilde{M} \subseteq \widetilde{K}$. Let $a_{t} \in A$ and $(\zeta, v) \in B$. Then one easily calculates that

$$
\begin{aligned}
\tilde{a}_{t}(\zeta, v)= & \mathcal{C}^{-1} \circ a_{t} \circ \mathcal{C}(\zeta, v) \\
= & || 1-\left.\zeta\right|^{2}+\left.t\left(1+2 \operatorname{Im} \zeta-|\zeta|^{2}\right)\right|^{-2} \\
& \times\left(-|1-\zeta|^{4}+4 t \operatorname{Im} \zeta+\left.\left.t^{2}|1+2 \operatorname{Im} \zeta-| \zeta\right|^{2}\right|^{2}\right. \\
& \left.4 t^{1 / 2}|1-\zeta|^{2}\left(|1-\zeta|^{2}+t\left(1-\operatorname{Im} \zeta-|\zeta|^{2}\right)\right)((1-\bar{\zeta}) v)\right)
\end{aligned}
$$

Suppose that $\widetilde{m}=(\varphi, \psi) \in \widetilde{M}$. Since $\varphi(e)=e$, we have that $\varphi(\bar{\zeta})=\overline{\varphi(\zeta)}$ and $\varphi(\operatorname{Im} \zeta)=$ $\operatorname{Im} \varphi(\zeta)$ for each $\zeta \in C$. Moreover, $|\zeta|=|\varphi(\zeta)|$ for each $\zeta \in C$. Then the first component of $\widetilde{m} \circ \widetilde{a}_{t}(\zeta, v)$ is given by

$$
\begin{aligned}
& \varphi\left(\frac{-|1-\zeta|^{4}+4 t \operatorname{Im} \zeta+\left.\left.t^{2}|1+2 \operatorname{Im} \zeta-| \zeta\right|^{2}\right|^{2}}{|| 1-\left.\zeta\right|^{2}+\left.t\left(1+2 \operatorname{Im} \zeta-|\zeta|^{2}\right)\right|^{2}}\right) \\
& \quad=\frac{-|1-\zeta|^{4}+4 t \operatorname{Im} \varphi(\zeta)+\left.\left.t^{2}|1+2 \operatorname{Im} \zeta-| \zeta\right|^{2}\right|^{2}}{|| 1-\left.\zeta\right|^{2}+\left.t\left(1+2 \operatorname{Im} \zeta-|\zeta|^{2}\right)\right|^{2}} \\
& =\frac{-|1-\varphi(\zeta)|^{4}+4 t \operatorname{Im} \varphi(\zeta)+\left.\left.t^{2}|1+2 \operatorname{Im} \varphi(\zeta)-| \varphi(\zeta)\right|^{2}\right|^{2}}{|| 1-\left.\varphi(\zeta)\right|^{2}+\left.t\left(1+2 \operatorname{Im} \varphi(\zeta)-|\varphi(\zeta)|^{2}\right)\right|^{2}}
\end{aligned}
$$

This is the first component of $\widetilde{a}_{t} \circ \widetilde{m}(\zeta, v)$. The second component of $\widetilde{m} \circ \widetilde{a}_{t}(\zeta, v)$ reads as

$$
\begin{aligned}
& \psi\left(\frac{\left(|1-\zeta|^{2}+t\left(1-2 \operatorname{Im} \zeta-|\zeta|^{2}\right)\right)((1-\bar{\zeta}) v)}{|| 1-\left.\zeta\right|^{2}+\left.t\left(1+2 \operatorname{Im} \zeta-|\zeta|^{2}\right)\right|^{2}}\right) \\
& =\frac{\varphi\left(|1-\zeta|^{2}+t\left(1-2 \operatorname{Im} \zeta-|\zeta|^{2}\right)\right) \psi((1-\bar{\zeta}) v)}{|| 1-\left.\zeta\right|^{2}+\left.t\left(1+2 \operatorname{Im} \zeta-|\zeta|^{2}\right)\right|^{2}} \\
& =\frac{\left(|1-\zeta|^{2}+t\left(1-2 \operatorname{Im} \varphi(\zeta)-|\zeta|^{2}\right)\right)(\varphi(1-\bar{\zeta}) \psi(v))}{|| 1-\left.\zeta\right|^{2}+\left.t\left(1+2 \operatorname{Im} \zeta-|\zeta|^{2}\right)\right|^{2}} \\
& =\frac{\left(|1-\varphi(\zeta)|^{2}+t\left(1-2 \operatorname{Im} \varphi(\zeta)-|\varphi(\zeta)|^{2}\right)\right)((1-\overline{\varphi(\zeta)} \psi(v))}{|| 1-\left.\varphi(\zeta)\right|^{2}+\left.t\left(1+2 \operatorname{Im} \varphi(\zeta)-|\varphi(\zeta)|^{2}\right)\right|^{2}}
\end{aligned}
$$


Clearly, this is the second component of $\widetilde{a}_{t} \circ \widetilde{m}(\zeta, v)$. Hence, $\widetilde{m}$ commutes with each $\widetilde{a}_{t}$. This shows that $\widetilde{M} \subseteq Z_{\widetilde{K}}(\widetilde{A})$.

Conversely, suppose that $\widetilde{m} \in Z_{\widetilde{K}}(\underset{\widetilde{A}}{\widetilde{M}}$. We have to show that $\widetilde{m}(1,0)=(1,0)$. Then [15, Proposition 4.1] implies that $\widetilde{m} \in \widetilde{M}$. For each $t \in \mathbb{R}^{+}$, we have

$$
\widetilde{a}_{t}(1,0)=\lim _{n \rightarrow \infty} \tilde{a}_{t}\left(1-\frac{1}{n}, 0\right)=(1,0) .
$$

Thus, $\widetilde{a}_{t}(\widetilde{m}(1,0))=\widetilde{m}(1,0)$. The only points on $\partial B$ that are invariant under each $\widetilde{a}_{t}$ are $(1,0)$ and $(-1,0)$. Seeking a contradiction assume that $\widetilde{m}(1,0)=(-1,0)$. Since $\widetilde{\sigma}=-\mathrm{id}$, we get $\widetilde{\sigma} \circ \widetilde{m}(1,0)=(1,0)$. By [15, Proposition 4.1] we have $\widetilde{\sigma} \circ \widetilde{m} \in \widetilde{M}$. Our previous argument then shows that $\widetilde{\sigma} \circ \widetilde{m}$ commutes with all $\widetilde{a}_{t}$. Therefore,

$$
\tilde{a}_{t} \circ \tilde{\sigma} \circ \tilde{m}=\tilde{\sigma} \circ \tilde{m} \circ \tilde{a}_{t}=\tilde{\sigma} \circ \tilde{a}_{t} \circ \tilde{m},
$$

which means that $\widetilde{\sigma}$ commutes with each $\widetilde{a}_{t}$. This is a contradiction. Hence it follows that $\widetilde{m}(1,0)=(1,0)$, which by $\left[15\right.$, Proposition 4.1] shows that $\widetilde{m} \in \widetilde{M}$. Therefore, $\widetilde{M}=Z_{\widetilde{K}}(\widetilde{A})$. Now let $\widetilde{m}=(\varphi, \psi) \in \widetilde{M}$ and set $m:=\mathcal{C} \circ \widetilde{m} \circ \mathcal{C}^{-1}$. For $(\eta, u) \in D$ we find

$$
\begin{aligned}
m(\eta, u) & =\mathcal{C} \circ \tilde{m} \circ \mathcal{C}^{-1}(\eta, u) \\
& =\mathcal{C} \circ \tilde{m}\left(|1+\eta|^{-2}\left(1+2 \operatorname{Im} \eta+|\eta|^{2}, 2(1+\bar{\eta}) u\right)\right) \\
& =\mathcal{C}\left(|1+\varphi(\eta)|^{-2}\left(1+2 \operatorname{Im} \varphi(\eta)+|\varphi(\eta)|^{2}, 2(1+\overline{\varphi(\eta)}) \psi(u)\right)\right) \\
& =(\varphi(\eta), \psi(u)) .
\end{aligned}
$$

The remaining claim follows directly from continuity or, alternatively, from the extension of the Cayley transform to $\bar{B}$.

\section{Isometric fundamental regions}

Throughout this section let $(C, e, V, J)$ be a $J^{2} C$-module structure such that $(C, V) \neq$ $(\mathbb{R} e,\{0\})$ and suppose that $(\mathfrak{z}, \mathfrak{v}, J)$ is the corresponding $H$-type algebra with $J^{2}$-condition. Recall the model $D$ of the rank one Riemannian symmetric space of noncompact type which is constructed from $(C, e, V, J)$, respectively, $(\mathfrak{z}, \mathfrak{v}, J)$ in Sect. 2.4. Let $G$ denote the full isometry group of $D$.

The purpose of this section is to prove the existence of isometric fundamental regions for certain subgroups $\Gamma$ of $G$. For this we first have to define the notion of the isometric sphere of $g \in G \backslash G_{\infty}$, which is a sphere w.r.t. Cygan metric. The Cygan metric is a metric on $\bar{D}^{g} \backslash\{\infty\}$ which arises from a certain group norm on $\bar{D}^{g} \backslash\{\infty\}$. This group norm is an extension of the Heisenberg pseudonorm.

\subsection{The Cauchy-Schwarz Theorem for $\beta_{2}$}

In our setting, the proof of the Cauchy-Schwarz Theorem as it is usually taught in linear algebra cannot be adapted to the map $\beta_{2}$. Therefore, we provide an alternative proof for which the following two lemmas are needed.

Lemma 3.1 Let $u, v \in V$. Then $\beta_{2}(v, u)=0$ if and only if $v \in(C u)^{\perp}$.

Proof We have $\beta_{2}(v, u)=0$ if and only if

$$
\left\langle\beta_{2}(v, u), \zeta\right\rangle=0 \text { for all } \zeta \in C \text {. }
$$


By the definition of $\beta_{2}$, this holds if and only if

$$
\langle\zeta u, v\rangle=0 \text { for all } \zeta \in C,
$$

hence if and only if $v \in(C u)^{\perp}$.

Lemma 3.2 Let $u \in V$ and $\lambda \in C$. Then $\beta_{2}(\lambda u, u)=|u|^{2} \lambda$.

Proof By the definition of $\beta_{2}$ and the polarization (2.6) we have

$$
\left\langle\beta_{2}(\lambda u, u), \zeta\right\rangle=\langle\zeta u, \lambda u\rangle=\langle\lambda, \zeta\rangle|u|^{2}=\left\langle|u|^{2} \lambda, \zeta\right\rangle
$$

for all $\zeta \in C$. Hence $\beta_{2}(\lambda u, u)=|u|^{2} \lambda$.

Proposition 3.3 Let $u, v \in V$. Then

$$
\left|\beta_{2}(u, v)\right| \leq|u||v| .
$$

Equality holds if and only if $v \in C u$ or $u \in C v$.

Proof Let $\left(v_{1}, v_{2}\right) \in C u \times(C u)^{\perp}$ be the unique pair such that $v=v_{1}+v_{2}$. Using that $\beta_{2}$ is $C$-hermitian (see Proposition 2.11), Lemma 3.1 yields

$$
\beta_{2}(u, v)=\beta_{2}\left(u, v_{1}\right)+\beta_{2}\left(u, v_{2}\right)=\beta_{2}\left(u, v_{1}\right) .
$$

Then Lemma 3.2 shows

$$
\left|\beta_{2}\left(u, v_{1}\right)\right|^{2}=|u|^{2}\left|v_{1}\right|^{2} .
$$

Clearly

$$
\left|v_{1}\right|^{2} \leq\left|v_{1}\right|^{2}+\left|v_{2}\right|^{2}=|v|^{2},
$$

where equality holds if and only if $v_{2}=0$, hence if and only if $v=v_{1} \in C u$. Thus,

$$
\left|\beta_{2}(u, v)\right|^{2}=\left|\beta_{2}\left(u, v_{1}\right)\right|^{2}=|u|^{2}\left|v_{1}\right|^{2} \leq|u|^{2}|v|^{2},
$$

where the inequality is an equality if and only if $u=0$ or $v \in C u$. This proves the claim.

\subsection{H-coordinates, Cygan metric, and isometric spheres}

Let $z=(t, Z, X) \in \bar{D}^{g} \backslash\{\infty\}$ and recall the map $\Theta$ from (2.4). The horospherical coordinates or $H$-coordinates of $z$ relative to the origin $o_{D}$ of $D$ are defined as

$$
\Theta^{-1}(z)=\left(t-\frac{1}{4}|X|^{2}, Z, X\right) .
$$

To avoid confusion with the ordinary coordinates of $\bar{D}^{g}$, H-coordinates will be subscripted by $h$. Hence, the H-coordinates of $z$ are denoted by $\left(t-\frac{1}{4}|X|^{2}, Z, X\right)_{h}$.

Since $\Theta$ induces a bijection between $\bar{D}^{g} \backslash\{\infty\}$ and the topological closure

$$
\bar{S}=\mathbb{R}_{0}^{+} \times \mathfrak{z} \times \mathfrak{v}
$$

of $S$ in $\mathbb{R} \times \mathfrak{z} \times \mathfrak{v}$, there is a geometric characterization of H-coordinates. We elaborate on this in the following remark. 
Remark and Definition 3.4 Let $z=(t, Z, X)=(\zeta, v) \in \bar{D}^{g} \backslash\{\infty\}$. The horosphere through $z$ with center $\infty$ is the $N$-orbit of $z$. We extend the group $A$ to the set

$$
A^{+}:=A \cup\left\{a_{0}\right\},
$$

where $a_{0}: \bar{D}^{g} \rightarrow \bar{D}^{g}$ is defined by $a_{0} \infty:=\infty$ and $a_{0} z:=0$ for all $z \in \bar{D}^{g} \backslash\{\infty\}$. Then there is a unique pair $\left(a_{s}, n\right) \in A^{+} \times N$ such that

$$
n a_{S}\left(o_{D}\right)=z \text {. }
$$

The height of $z$ is defined as

$$
\operatorname{ht}(z):=t-\frac{1}{4}|X|^{2}=\operatorname{Re} \zeta-\frac{1}{4}|v|^{2} .
$$

Formula (2.5) shows that $s=\mathrm{ht}(z)$ and $n=(1, Z, X)$. Hence the H-coordinates of $z$ are

$$
(\operatorname{ht}(z), Z, X)_{h}=(\operatorname{ht}(z), \operatorname{Im} \zeta, v)_{h} \text {. }
$$

This means that the H-coordinates are given by the height of the $\infty$-centered horosphere on which $z$ lies and the coordinates of $z$ in the canonical parametrization of this horosphere.

The Cygan metric on $D$ is a metric on $\bar{D}^{g} \backslash\{\infty\}$ which arises from a certain group norm. If $(\mathcal{G}, \cdot)$ is a group with neutral element $1_{\mathcal{G}}$, then a map $p: \mathcal{G} \rightarrow \mathbb{R}_{0}^{+}$is called a group norm if

(GN1) $p(g)=0$ if and only if $g=1_{\mathcal{G}}$,

(GN2) $p\left(g^{-1}\right)=p(g)$ for all $g \in \mathcal{G}$,

(GN3) $p(g h) \leq p(g)+p(h)$ for all $g, h \in \mathcal{G}$.

Suppose that $p$ is a group norm on $\mathcal{G}$, then the map $d: \mathcal{G} \times \mathcal{G} \rightarrow \mathbb{R}, d(g, h):=p\left(g^{-1} h\right)$ is a metric on $\mathcal{G}$. It is called the metric induced by $p$.

A well-known example of a group norm and a pre-form of the group norm for the Cygan metric is the Heisenberg pseudonorm on $N$. More precisely, $N$ inherits an inner product from its canonical bijection to $\mathfrak{z} \times \mathfrak{v}$. Then the Heisenberg group norm $q$ (which is also known as the Heisenberg pseudonorm) on $N$ is defined by

$$
q(Z, X):=\left.\left|\frac{1}{4}\right| X\right|^{2}+\left.Z\right|^{1 / 2}=\left(\frac{1}{16}|X|^{4}+|Z|^{2}\right)^{1 / 4} .
$$

The last equality holds because $\mathfrak{z}$ and $\mathbb{R} \cong \mathfrak{a}$ are orthogonal. Since each height level set of $\bar{D}^{g} \backslash\{\infty\}$ is isomorphic to $N$, the metric induced from the Heisenberg group norm measures the distance between two elements in the same height level set.

To be able to also measure the distance between elements in different height level sets, we extend the Heisenberg pseudonorm to the direct product of the groups $(\mathbb{R},+$ ) ("differences between height level sets") and $N$ by

$$
p:\left\{\begin{array}{l}
\mathbb{R} \times N \rightarrow \mathbb{R} \\
\left.(k, Z, X) \mapsto\left|\frac{1}{4}\right| X\right|^{2}+|k|+\left.Z\right|^{1 / 2} .
\end{array}\right.
$$

Obviously, $\left.p\right|_{\{0\} \times N}=q$.

Proposition 3.5 The map $p$ is a group norm on $\mathbb{R} \times N$. 
Proof The neutral element of $\mathbb{R} \times N$ is $(0,0,0)$, and $\mathfrak{z}$ is orthogonal to $\mathbb{R}$. This implies that $p$ satisfies (GN1). To prove (GN2) let $g=(k, Z, X) \in \mathbb{R} \times N$. Then

$$
\begin{aligned}
p\left(g^{-1}\right) & =p(-k,-Z,-X)=\left.\left|\frac{1}{4}\right| X\right|^{2}+|k|-\left.Z\right|^{1 / 2}=\left(\left(\frac{1}{4}|X|^{2}+|k|\right)^{2}+|Z|^{2}\right)^{1 / 4} \\
& =\left.\left|\frac{1}{4}\right| X\right|^{2}+|k|+\left.Z\right|^{1 / 2}=p(g) .
\end{aligned}
$$

The triangle equality (GN3) is shown in several steps. For each $g=(k, Z, X) \in \mathbb{R} \times N$ we have

$$
\begin{aligned}
p(g) & =\left.\left|\frac{1}{4}\right| X\right|^{2}+|k|+\left.Z\right|^{1 / 2}=\left(\left(\frac{1}{4}|X|^{2}+|k|\right)^{2}+|Z|^{2}\right)^{1 / 4} \\
& \geq\left(\left(\frac{1}{4}|X|^{2}\right)^{2}\right)^{1 / 4}=\frac{1}{2}|X| .
\end{aligned}
$$

This and Proposition 3.3 yield that

$$
\frac{1}{4}\left|\beta_{2}\left(X_{2}, X_{1}\right)\right| \leq \frac{\left|X_{1}\right|}{2} \frac{\left|X_{2}\right|}{2} \leq p\left(k_{1}, Z_{1}, X_{1}\right) p\left(k_{2}, Z_{2}, X_{2}\right)
$$

for all $\left(k_{j}, Z_{j}, X_{j}\right) \in \mathbb{R} \times N, j=1,2$. Further, for all $(Z, X) \in N$ and $k_{1}, k_{2} \in \mathbb{R}$, we find

$$
\begin{aligned}
\left.\left|\frac{1}{4}\right| X\right|^{2}+\left|k_{1}+k_{2}\right|+\left.Z\right|^{1 / 2} & =\left(\left(\frac{1}{4}|X|^{2}+\left|k_{1}+k_{2}\right|\right)^{2}+|Z|^{2}\right)^{1 / 4} \\
& \leq\left(\left(\frac{1}{4}|X|^{2}+\left|k_{1}\right|+\left|k_{2}\right|\right)^{2}+|Z|^{2}\right)^{1 / 4} \\
& =\left.\left|\frac{1}{4}\right| X\right|^{2}+\left|k_{1}\right|+\left|k_{2}\right|+\left.Z\right|^{1 / 2} .
\end{aligned}
$$

Now let $g_{j}=\left(k_{j}, Z_{j}, X_{j}\right) \in \mathbb{R} \times N, j=1,2$. Then

$$
\begin{aligned}
p\left(g_{1} g_{2}\right) & =p\left(k_{1}+k_{2}, Z_{1}+Z_{2}+\frac{1}{2}\left[X_{1}, X_{2}\right], X_{1}+X_{2}\right) \\
& =\left|\frac{1}{4}\right| X_{1}+\left.X_{2}\right|^{2}+\left|k_{1}+k_{2}\right|+Z_{1}+Z_{2}+\left.\frac{1}{2}\left[X_{1}, X_{2}\right]\right|^{1 / 2} \\
& \leq\left|\frac{1}{4}\right| X_{1}+\left.X_{2}\right|^{2}+\left|k_{1}\right|+\left|k_{2}\right|+Z_{1}+Z_{2}+\left.\frac{1}{2}\left[X_{1}, X_{2}\right]\right|^{1 / 2} \\
& =\left.\left|\frac{1}{4}\right| X_{1}\right|^{2}+\left|k_{1}\right|+Z_{1}+\frac{1}{4}\left|X_{2}\right|^{2}+\left|k_{2}\right|+Z_{2}+\left.\frac{1}{2}\left(\left\langle X_{1}, X_{2}\right\rangle+\left[X_{1}, X_{2}\right]\right)\right|^{1 / 2} .
\end{aligned}
$$

Recall from Lemma 2.12 that

$$
\left\langle X_{1}, X_{2}\right\rangle+\left[X_{1}, X_{2}\right]=\beta_{2}\left(X_{2}, X_{1}\right) .
$$


Then

$$
\begin{aligned}
p\left(g_{1} g_{2}\right) & \leq\left.\left|\frac{1}{4}\right| X_{1}\right|^{2}+\left|k_{1}\right|+Z_{1}+\frac{1}{4}\left|X_{2}\right|^{2}+\left|k_{2}\right|+Z_{2}+\left.\frac{1}{2} \beta_{2}\left(X_{2}, X_{1}\right)\right|^{1 / 2} \\
& \leq\left[\left.\left|\frac{1}{4}\right| X_{1}\right|^{2}+\left|k_{1}\right|+Z_{1}|+| \frac{1}{4}\left|X_{2}\right|^{2}+\left|k_{2}\right|+Z_{2}\left|+\frac{1}{2}\right| \beta_{2}\left(X_{2}, X_{1}\right) \mid\right]^{1 / 2} \\
& =\left[p\left(g_{1}\right)^{2}+p\left(g_{2}\right)^{2}+\frac{1}{2}\left|\beta_{2}\left(X_{2}, X_{1}\right)\right|\right]^{1 / 2} \\
& \leq\left[p\left(g_{1}\right)^{2}+p\left(g_{2}\right)^{2}+2 p\left(g_{1}\right) p\left(g_{2}\right)\right]^{1 / 2} \\
& =p\left(g_{1}\right)+p\left(g_{2}\right) .
\end{aligned}
$$

This completes the proof.

The definition of the Cygan metric uses the H-coordinates on $\bar{D}^{g} \backslash\{\infty\}$. We use the map

$$
\kappa:\left\{\begin{aligned}
\bar{D}^{g} \backslash\{\infty\} & \rightarrow \mathbb{R} \times N \\
z & \mapsto \Theta^{-1}(z)
\end{aligned}\right.
$$

to assign to each element of $\bar{D}^{g} \backslash\{\infty\}$ its H-coordinates. The advantage of using H-coordinates is that the points of $\bar{D}^{g} \backslash\{\infty\}$ get embedded into the group $\mathbb{R} \times N$. This in turn allows to perform the group operations of $\mathbb{R} \times N$ on $\kappa\left(z_{1}\right), \kappa\left(z_{2}\right)$ for elements $z_{1}, z_{2} \in \bar{D}^{g} \backslash\{\infty\}$.

Definition 3.6 The Cygan metric on $D$ is given by

$$
\rho:\left\{\begin{aligned}
\bar{D}^{g} \backslash\{\infty\} \times \bar{D}^{g} \backslash\{\infty\} & \rightarrow \mathbb{R} \\
\left(g_{1}, g_{2}\right) & \mapsto p\left(\kappa\left(g_{1}\right)^{-1} \kappa\left(g_{2}\right)\right) .
\end{aligned}\right.
$$

Since $\kappa$ is injective, the Cygan metric is in fact a metric on $\bar{D}^{g} \backslash\{\infty\}$.

The following lemma provides worked out formulas for the Cygan metric. It can be proved by a straightforward calculation.

Lemma 3.7 Let $z_{j}=\left(\zeta_{j}, v_{j}\right)=\left(k_{j}, Z_{j}, X_{j}\right)_{h}$ be elements of $\bar{D}^{g} \backslash\{\infty\}$. Then the Cygan metric is given by

$$
\begin{aligned}
\rho\left(z_{1}, z_{2}\right) & =\left|\frac{1}{4}\right| X_{1}-\left.X_{2}\right|^{2}+\left|k_{1}-k_{2}\right|+Z_{1}-Z_{2}+\left.\frac{1}{2} \operatorname{Im} \beta_{2}\left(X_{2}, X_{1}\right)\right|^{1 / 2} \\
& =\left.\left|\frac{1}{4}\right| v_{1}\right|^{2}+\frac{1}{4}\left|v_{2}\right|^{2}+\left|\operatorname{ht}\left(z_{1}\right)-\operatorname{ht}\left(z_{2}\right)\right|+\operatorname{Im} \zeta_{1}-\operatorname{Im} \zeta_{2}-\left.\frac{1}{2} \beta_{2}\left(v_{1}, v_{2}\right)\right|^{1 / 2} .
\end{aligned}
$$

Convention 3.8 Let $g \in G \backslash G_{\infty}$. Whenever in the following we write $g=n_{1} \sigma m a_{t} n_{2}$, it should be understood that $n_{1}, n_{2} \in N, a_{t} \in A$ and $m \in M$.

Definition 3.9 Let $g \in G \backslash G_{\infty}$. Suppose that $g=n_{1} \sigma m a_{t} n_{2}$, and let $R(g):=t^{-1 / 4}$. Then the set

$$
I(g):=\left\{z \in D \mid \rho\left(z, g^{-1} \infty\right)=R(g)\right\}
$$

is called the isometric sphere of $g$. Further,

$$
\operatorname{ext} I(g):=\left\{z \in D \mid \rho\left(z, g^{-1} \infty\right)>R(g)\right\}
$$


is called the exterior of $I(g)$, and

$$
\text { int } I(g):=\left\{z \in D \mid \rho\left(z, g^{-1} \infty\right)<R(g)\right\}
$$

is called the interior of $I(g)$. The value $R(g)$ is the radius of $I(g)$.

Lemma 3.10 Let $g=n_{1} \sigma m a_{t} n_{2} \in G \backslash G_{\infty}$ and $n_{2}=\left(1, Z_{s 2}, X_{s 2}\right)$. Then

$$
\rho\left((\zeta, v), g^{-1} \infty\right)=\left.\left|\frac{1}{4}\right| X_{s 2}\right|^{2}+Z_{s 2}+\zeta+\left.\frac{1}{2} \beta_{2}\left(v, X_{s 2}\right)\right|^{1 / 2} .
$$

Further $\rho\left(\cdot, g^{-1} \infty\right)$ is unbounded on $D$, and hence $\operatorname{ext} I(g) \neq \emptyset$.

Proof We have

$$
g^{-1} \infty=n_{2}^{-1} 0=\left(\frac{1}{4}\left|X_{s 2}\right|^{2}-Z_{s 2},-X_{s 2}\right) .
$$

Thus, the expression for $\rho\left((\zeta, v), g^{-1} \infty\right)$ follows immediately from Lemma 3.7. We now prove the second part of the claim. Let $(\zeta, v) \in D$ and $t>1$. Then

$$
\text { ht }((t \zeta, v))=t \operatorname{Re} \zeta-\frac{1}{4}|v|^{2}>\operatorname{Re} \zeta-\frac{1}{4}|v|^{2}>0 .
$$

Hence $(t \zeta, v) \in D$. Further

$$
\begin{aligned}
\rho\left((t \zeta, v), g^{-1} \infty\right)^{2} & =\left.\left|t \zeta+\frac{1}{4}\right| X_{s 2}\right|^{2}+Z_{s 2}+\frac{1}{2} \beta_{2}\left(v, X_{s 2}\right) \mid \\
& \geq t|\zeta|-\left.\left|\frac{1}{4}\right| X_{s 2}\right|^{2}+Z_{s 2}+\frac{1}{2} \beta_{2}\left(v, X_{s 2}\right) \mid,
\end{aligned}
$$

which converges to $\infty$ for $t \rightarrow \infty$. Hence $\rho\left(\cdot, g^{-1} \infty\right)$ is unbounded. This completes the proof.

Lemma 3.11 Let $g \in G \backslash G_{\infty}$. Then

(i) ext $I(g)$ and int $I(g)$ are open,

(ii) $\overline{\operatorname{ext} I(g)}=\left\{z \in D \mid \rho\left(z, g^{-1} \infty\right) \geq R(g)\right\}=\complement$ int $I(g)$,

(iii) $\overline{\text { int } I(g)}=\left\{z \in D \mid \rho\left(z, g^{-1} \infty\right) \leq R(g)\right\}=\complement \operatorname{ext} I(g)$.

(iv) If $(\zeta, v) \in \overline{\text { int } I(g)}$, then $(\zeta-s, v) \in \operatorname{int} I(g)$ for each $s \in(0, \operatorname{ht}(\zeta))$.

(v) If $(\zeta, v) \in \overline{\operatorname{ext} I(g)}$, then $(\zeta+s, v) \in \operatorname{ext} I(g)$ for each $s>0$.

Proof Suppose that $g=n_{1} \sigma m a_{t} n_{2}$ with $n_{2}=\left(1, Z_{2}, X_{2}\right)$. Then $R(g)=t^{-1 / 4}$ and, by Lemma 3.10,

$$
\rho\left((\zeta, v), g^{-1} \infty\right)=\left.\left|\frac{1}{4}\right| X_{2}\right|^{2}+Z_{2}+\zeta+\left.\frac{1}{2} \beta_{2}\left(v, X_{2}\right)\right|^{1 / 2}
$$

for each $(\zeta, v) \in D$. Since $\beta_{2}\left(\cdot, X_{2}\right): V \rightarrow C$ is $\mathbb{R}$-linear (see Proposition 2.11), the map

$$
f: \begin{cases}D & \rightarrow \mathbb{R} \\ (\zeta, v) & \left.\mapsto\left|\frac{1}{4}\right| X_{2}\right|^{2}+Z_{2}+\zeta+\frac{1}{2} \beta_{2}\left(v, X_{2}\right) \mid\end{cases}
$$

is continuous. Then

$$
\operatorname{ext} I(g)=f^{-1}\left(\left(t^{-1 / 2}, \infty\right)\right) \text { and int } I(g)=f^{-1}\left(\left(-\infty, t^{-1 / 2}\right)\right) .
$$


This shows that ext $I(g)$ and int $I(g)$ are open. Moreover, it follows that $\overline{\text { int } I(g)}$ is contained in $f^{-1}\left(\left(-\infty, t^{-1 / 2}\right]\right)$. For the converse inclusion relation, it suffices to show that $f^{-1}\left(t^{-1 / 2}\right) \subseteq \overline{\operatorname{int} I(g)}$. Let $z_{0}=\left(\zeta_{0}, v_{0}\right) \in f^{-1}\left(t^{-1 / 2}\right)$. Then

$$
\begin{aligned}
t^{-1 / 2} & =\left.\left|\frac{1}{4}\right| X_{2}\right|^{2}+Z_{2}+\zeta_{0}+\frac{1}{2} \beta_{2}\left(v_{0}, X_{2}\right) \mid \\
& =\left[\left|\frac{1}{4}\right| X_{2}+\left.v_{0}\right|^{2}+\left.\operatorname{ht}\left(\zeta_{0}\right)\right|^{2}+\left|Z_{2}+\operatorname{Im} \zeta_{0}+\frac{1}{2} \operatorname{Im} \beta_{2}\left(v_{0}, X_{2}\right)\right|^{2}\right]^{1 / 2} .
\end{aligned}
$$

For each $s \in\left(0, \operatorname{ht}\left(\zeta_{0}\right)\right)$ it follows that

$$
\begin{aligned}
t^{-1 / 2} & >\left[\left|\frac{1}{4}\right| X_{2}+\left.v_{0}\right|^{2}+\operatorname{ht}\left(\zeta_{0}\right)-\left.s\right|^{2}+\left|Z_{2}+\operatorname{Im} \zeta_{0}+\frac{1}{2} \operatorname{Im} \beta_{2}\left(v_{0}, X_{2}\right)\right|^{2}\right]^{1 / 2} \\
& =\left.\left|\frac{1}{4}\right| X_{2}\right|^{2}+Z_{2}+\zeta_{0}-s+\frac{1}{2} \beta_{2}\left(v_{0}, X_{2}\right) \mid .
\end{aligned}
$$

Thus, $\left(\zeta_{0}-s, v_{0}\right) \in \operatorname{int} I(g)$ for each $s \in\left(0, \operatorname{ht}\left(\zeta_{0}\right)\right)$. Hence

$$
\lim _{s \searrow 0}\left(\zeta_{0}-s, v_{0}\right)=\left(\zeta_{0}, v_{0}\right) \in \overline{\operatorname{int} I(g)}
$$

This proves (iii) and (iv). The proof of (ii) is analogous to that of (iii), and the proof of (v) is analogous to that of (iv).

Proposition 3.12 We have

$$
\bigcap_{g \in \Gamma \backslash \Gamma_{\infty}} \operatorname{ext} I(g)=\bigcap_{g \in \Gamma \backslash \Gamma_{\infty}} \overline{\operatorname{ext} I(g)}=D \backslash \bigcup_{g \in \Gamma \backslash \Gamma_{\infty}} \text { int } I(g) .
$$

Proof Lemma 3.11(iii) states that $\complement$ ext $I(g)=\overline{\operatorname{int} I(g)}$ for each $g \in \Gamma \backslash \Gamma_{\infty}$. Therefore,

$$
\complement\left(\overline{\bigcap_{g \in \Gamma \backslash \Gamma_{\infty}} \operatorname{ext} I(g)}\right)=\left(\bigcup_{g \in \Gamma \backslash \Gamma_{\infty}} \complement \operatorname{ext} I(g)\right)^{\circ}=\left(\bigcup_{g \in \Gamma \backslash \Gamma_{\infty}} \overline{\operatorname{int} I(g)}\right)^{\circ} .
$$

By Lemma 3.11(i), the interior of each isometric sphere is open. Thus

$$
\bigcup_{g \in \Gamma \backslash \Gamma_{\infty}} \operatorname{int} I(g)=\left(\bigcup_{g \in \Gamma \backslash \Gamma_{\infty}} \operatorname{int} I(g)\right)^{\circ}
$$

and hence

$$
\bigcup_{g \in \Gamma \backslash \Gamma_{\infty}} \operatorname{int} I(g) \subseteq\left(\bigcup_{g \in \Gamma \backslash \Gamma_{\infty}} \overline{\operatorname{int} I(g)}\right)^{\circ}
$$

In the following we will prove the converse inclusion relation. Let $z=(\zeta, v)$ be an element of $\left(\bigcup\left\{\overline{\operatorname{int} I(g)} \mid g \in \Gamma \backslash \Gamma_{\infty}\right\}\right)^{\circ}$ and pick $\varepsilon>0$ such that

$$
z_{\varepsilon}:=z+\varepsilon=(\zeta+\varepsilon, v) \in \bigcup_{g \in \Gamma \backslash \Gamma_{\infty}} \overline{\operatorname{int} I(g)} .
$$


Fix some $k \in \Gamma \backslash \Gamma_{\infty}$ such that $z_{\varepsilon} \in \overline{\operatorname{int} I(k)}$. Lemma 3.11(iv) yields that $z=z_{\varepsilon}-\varepsilon$ is in int $I(k)$. Thus $z \in \bigcup\left\{\right.$ int $\left.I(g) \mid g \in \Gamma \backslash \Gamma_{\infty}\right\}$. This shows that

$$
\left(\bigcup_{g \in \Gamma \backslash \Gamma_{\infty}} \overline{\operatorname{int} I(g)}\right)^{\circ} \subseteq \bigcup_{g \in \Gamma \backslash \Gamma_{\infty}} \operatorname{int} I(g) .
$$

In turn,

$$
\complement \bigcup_{g \in \Gamma \backslash \Gamma_{\infty}} \operatorname{int} I(g)=\overline{\bigcap_{g \in \Gamma \backslash \Gamma_{\infty}} \operatorname{ext} I(g)} \text {. }
$$

Finally we have $\complement$ int $I(g)=\overline{\operatorname{ext} I(g)}$ for each $g \in \Gamma \backslash \Gamma_{\infty}$ by Lemma 3.11(iii). Therefore,

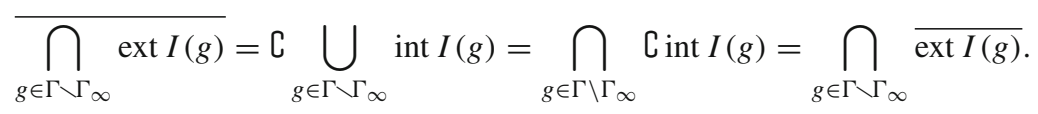

Let $g \in G \backslash G_{\infty}$ and $(\zeta, v) \in \bar{D}^{g} \backslash\{\infty\}$. Suppose that $g=n_{1} \sigma m a_{t} n_{2}$ with $n_{j}=\left(1, Z_{s j}, X_{s j}\right)$ and $m=(\varphi, \psi)$. A lengthy but easy calculation shows that

$$
\begin{gathered}
g(\zeta, v)=\left(\frac{1}{4}\left|X_{s 1}\right|^{2}+Z_{s 1}+x t^{-1}-\frac{1}{2} t^{-1 / 2} \beta_{2}\left(x \psi\left(X_{s 2}+v\right), X_{s 1}\right),\right. \\
\left.X_{s 1}-x t^{-1 / 2} \psi\left(X_{s 2}+v\right)\right)
\end{gathered}
$$

where

$$
x:=\left[\varphi\left(\frac{1}{4}\left|X_{s 2}\right|^{2}+Z_{s 2}+\zeta+\frac{1}{2} \beta_{2}\left(v, X_{s 2}\right)\right)\right]^{-1} .
$$

This explicit expression for the action of $g$ on $D$ is used in the following two lemmas. We denote the set of orthogonal endomorphisms of $C$, respectively of $V$, by $O(C)$, respectively $O(V)$.

Lemma 3.13 If $g \in G \backslash G_{\infty}$, then $g$ maps ext $I(g)$ onto int $I\left(g^{-1}\right)$, and $I(g)$ onto $I\left(g^{-1}\right)$.

Proof Suppose that $g=n_{1} \sigma m a_{t} n_{2}$ with $n_{j}=\left(1, Z_{s j}, X_{s j}\right)$ and $m=(\varphi, \psi)$. Then we have $g^{-1}=n_{2}^{-1} \sigma m a_{t} n_{1}^{-1}$. Hence it follows that

$$
R(g)=R\left(g^{-1}\right)=t^{-1 / 4}
$$

and

$$
g^{-1} I\left(g^{-1}\right)=\{z \in D \mid \rho(g z, g \infty)=R(g)\} .
$$

In the following we will compare $\rho(g z, g \infty)$ to $\rho\left(z, g^{-1} \infty\right)$. Let $(\zeta, v)$ be in $D$. Set

$$
x:=\left[\varphi\left(\frac{1}{4}\left|X_{s 2}\right|^{2}+Z_{s 2}+\zeta+\frac{1}{2} \beta_{2}\left(v, X_{s 2}\right)\right)\right]^{-1} .
$$

Then (3.1) and Lemma 3.10 yield that

$$
\rho(g(\zeta, v), g \infty)=\left.\left|\frac{1}{2}\right| X_{s 1}\right|^{2}+x t^{-1}-\left.\frac{1}{2} \beta_{2}\left(X_{s 1}, X_{s 1}\right)\right|^{1 / 2} .
$$


By Proposition 2.11, $\beta_{2}\left(X_{s 1}, X_{s 1}\right)=\left|X_{s 1}\right|^{2}$. Thus

$$
\rho(g(\zeta, v), g \infty)=\left|x t^{-1}\right|^{1 / 2}=|x|^{1 / 2} t^{-1 / 2} .
$$

Since $\varphi \in O(C)$, it follows that

$$
\begin{aligned}
|x|^{1 / 2} & =\left|\varphi\left(\frac{1}{4}\left|X_{s 2}\right|^{2}+Z_{s 2}+\zeta+\frac{1}{2} \beta_{2}\left(v, X_{s 2}\right)\right)\right|^{-1 / 2} \\
& =\left.\left|\frac{1}{4}\right| X_{s 2}\right|^{2}+Z_{s 2}+\zeta+\left.\frac{1}{2} \beta_{2}\left(v, X_{s 2}\right)\right|^{-1 / 2} \\
& =\rho\left((\zeta, v), g^{-1} \infty\right)^{-1}
\end{aligned}
$$

Therefore,

$$
\rho\left((\zeta, v), g^{-1} \infty\right) \rho(g(\zeta, v), g \infty)=t^{-1 / 2} .
$$

Hence, $(\zeta, v) \in I(g)$ if and only if

$$
\rho(g(\zeta, v), g \infty)=t^{-1 / 4} .
$$

This is equivalent to $g(\zeta, v) \in I\left(g^{-1}\right)$. In turn, $g I(g)=I\left(g^{-1}\right)$. Further, we have $(\zeta, v) \in$ ext $I(g)$ if and only if

$$
t^{-1 / 4}>\rho(g(\zeta, v), g \infty) .
$$

Therefore, $g$ ext $I(g)=$ int $I\left(g^{-1}\right)$.

Lemma 3.14 Let $n=(1, Z, X) \in N, a_{s} \in A$, and $m=(\varphi, \psi) \in M$.

(i) If $n^{\prime}:=\left(1, s Z, s^{1 / 2} X\right)$, then $a_{s} n=n^{\prime} a_{s}$.

(ii) For all $u, v \in V$ we have $\varphi\left(\beta_{2}(v, u)\right)=\beta_{2}(\psi(v), \psi(u))$.

(iii) If $n^{\prime}:=(1, \varphi(Z), \psi(X))$, then $m n=n^{\prime} m$.

Proof Claim (i) is shown by direct calculation. To prove (ii) recall that $\varphi \in O(C), \psi \in O(V)$, and that $\psi(J(\eta, w))=J(\varphi(\eta), \psi(w))$ for each $(\eta, w) \in C \oplus V$. For each $\zeta \in C$ we have

$$
\begin{aligned}
\left\langle\varphi\left(\beta_{2}(v, u)\right), \zeta\right\rangle & =\left\langle\beta_{2}(v, u), \varphi^{-1}(\zeta)\right\rangle=\left\langle J\left(\varphi^{-1}(\zeta), u\right), v\right\rangle \\
& =\left\langle\psi\left(J\left(\varphi^{-1}(\zeta), u\right)\right), \psi(v)\right\rangle=\langle J(\zeta, \psi(u)), \psi(v)\rangle \\
& =\left\langle\beta_{2}(\psi(v), \psi(u)), \zeta\right\rangle .
\end{aligned}
$$

Thus $\varphi\left(\beta_{2}(v, u)\right)=\beta_{2}(\psi(v), \psi(u))$. For the proof of (iii) let $(\zeta, v) \in D$. Then

$$
\begin{aligned}
m(n(\zeta, v)) & =\left(\varphi\left(\frac{1}{4}|X|^{2}+Z+\zeta+\frac{1}{2} \beta_{2}(v, X)\right), \psi(X+v)\right) \\
& =\left(\frac{1}{4}|\psi(X)|^{2}+\varphi(Z)+\varphi(\zeta)+\frac{1}{2} \varphi\left(\beta_{2}(v, X)\right), \psi(X)+\psi(v)\right) .
\end{aligned}
$$

Now (ii) yields

$$
\begin{aligned}
m(n(\zeta, v)) & =\left(\frac{1}{4}|\psi(X)|^{2}+\varphi(Z)+\varphi(\zeta)+\frac{1}{2} \beta_{2}(\psi(v), \psi(X)), \psi(X)+\psi(v)\right) \\
& =n^{\prime}(\varphi(\zeta), \psi(v))=n^{\prime}(m(\zeta, v)) .
\end{aligned}
$$

Hence $m n=n^{\prime} m$. 
Proposition 3.15 Let $g \in G_{\infty}$ and $h \in G \backslash G_{\infty}$. Then

$$
\begin{aligned}
g I(h) & =I\left(g h g^{-1}\right), \\
g \operatorname{int} I(h) & =\operatorname{int} I\left(g h g^{-1}\right), \\
g \operatorname{ext} I(h) & =\operatorname{ext}\left(g h g^{-1}\right) .
\end{aligned}
$$

Proof It suffices to prove the claim for the three cases $g \in A, g \in N$, and $g \in M$. This we will do separately. Let $h=n_{1} \sigma m a_{t} n_{2}$ with $n_{j}=\left(1, Z_{j}, X_{j}\right)$.

Suppose first that $g=a_{s}$. Let $(\zeta, v) \in D$. For $j \in\{1,2\}$ set $n_{j}^{\prime}:=\left(1, s Z_{j}, s^{1 / 2} X_{j}\right)$. Lemma 3.14 implies that

$$
g h g^{-1}=a_{s} n_{1} \sigma m a_{t} n_{2} a_{s}^{-1}=n_{1}^{\prime} \sigma m a_{s}^{-1} a_{t} a_{s}^{-1} n_{2}^{\prime}=n_{1}^{\prime} \sigma m a_{s^{-2} t} n_{2}^{\prime} .
$$

Therefore, $R\left(g h g^{-1}\right)=s^{1 / 2} t^{-1 / 4}=s^{1 / 2} R(h)$. By Lemma 3.10 we have

$$
\rho\left((\zeta, v),\left(g h g^{-1}\right)^{-1} \infty\right)=\left.\left|\frac{1}{4} s\right| X_{2}\right|^{2}+s Z_{2}+\zeta+\left.\frac{1}{2} s^{1 / 2} \beta_{2}\left(v, X_{2}\right)\right|^{1 / 2}
$$

and

$$
\begin{aligned}
\rho\left(a_{s}^{-1}(\zeta, v), h^{-1} \infty\right) & =\left.\left|\frac{1}{4}\right| X_{2}\right|^{2}+Z_{2}+s^{-1} \zeta+\left.\frac{1}{2} s^{-1 / 2} \beta_{2}\left(v, X_{2}\right)\right|^{1 / 2} \\
& =s^{-1 / 2} \rho\left((\zeta, v),\left(g h g^{-1}\right)^{-1} \infty\right) .
\end{aligned}
$$

Then

$$
\begin{aligned}
g I(h) & =\left\{z \in D \mid \rho\left(g^{-1} z, h^{-1} \infty\right)=R(h)\right\} \\
& =\left\{z \in D \mid \rho\left(z,\left(g h g^{-1}\right)^{-1} \infty\right)=s^{1 / 2} R(h)\right\} \\
& =\left\{z \in D \mid \rho\left(z,\left(g h g^{-1}\right)^{-1} \infty\right)=R\left(g h g^{-1}\right)\right\} \\
& =I\left(g h g^{-1}\right) .
\end{aligned}
$$

Analogously, we see that $g$ ext $I(h)=\operatorname{ext} I\left(g h g^{-1}\right)$ and $g$ int $I(h)=$ int $I\left(g h g^{-1}\right)$.

Now suppose that $g=n_{3}=\left(1, Z_{3}, X_{3}\right)$. Then $g h g^{-1}=\left(n_{3} n_{1}\right) \sigma m a_{t}\left(n_{2} n_{3}^{-1}\right)$ and (see Lemma 2.12)

$$
n_{2} n_{3}^{-1}=\left(1, Z_{2}-Z_{3}-\frac{1}{2} \operatorname{Im} \beta_{2}\left(X_{3}, X_{2}\right), X_{2}-X_{3}\right) .
$$

Therefore, by Lemmas 3.10 and 2.12,

$$
\begin{aligned}
& \rho\left((\zeta, v),\left(n_{3} h n_{3}^{-1}\right)^{-1} \infty\right) \\
& =\left.\left|\frac{1}{4}\right| X_{2}\right|^{2}+\frac{1}{4}\left|X_{3}\right|^{2}-\frac{1}{2} \beta_{2}\left(X_{3}, X_{2}\right)+Z_{2}-Z_{3}+\zeta+\left.\frac{1}{2} \beta_{2}\left(v, X_{2}-X_{3}\right)\right|^{1 / 2} .
\end{aligned}
$$


On the other hand, we have

$$
\begin{aligned}
\rho & \left(n_{3}^{-1}(\zeta, v), h^{-1} \infty\right) \\
& =\left.\left|\frac{1}{4}\right| X_{2}\right|^{2}+Z_{2}+\frac{1}{4}\left|X_{3}\right|^{2}-Z_{3}+\zeta-\frac{1}{2} \beta_{2}\left(v, X_{3}\right)+\left.\frac{1}{2} \beta_{2}\left(-X_{3}+v, X_{2}\right)\right|^{1 / 2} \\
& =\left.\left|\frac{1}{4}\right| X_{2}\right|^{2}+\frac{1}{4}\left|X_{3}\right|^{2}+Z_{2}-Z_{3}+\zeta-\frac{1}{2} \beta_{2}\left(X_{3}, X_{2}\right)+\left.\frac{1}{2} \beta_{2}\left(v, X_{2}-X_{3}\right)\right|^{1 / 2} \\
& =\rho\left((\zeta, v), n_{3} h^{-1} n_{3}^{-1} \infty\right) .
\end{aligned}
$$

Since $R(h)=R\left(g h g^{-1}\right)$, the claim follows for this case.

Finally suppose that $g=m_{2}=(\varphi, \psi)$ and set $n_{j}^{\prime}:=\left(1, \varphi\left(Z_{j}\right), \psi\left(X_{j}\right)\right)$ for $j=1,2$. Lemma 3.14 shows that

$$
g h g^{-1}=m_{2} n_{1} \sigma m a_{t} n_{2} m_{2}^{-1}=n_{1}^{\prime} \sigma m_{2}^{-1} m m_{2}^{-1} a_{t} n_{2}^{\prime} .
$$

From this it follows that $R(h)=R\left(m_{2} h m_{2}^{-1}\right)$. Moreover, we have

$$
\rho\left((\zeta, v),\left(g h g^{-1}\right)^{-1} \infty\right)=\left.\left|\frac{1}{4}\right| \psi\left(X_{2}\right)\right|^{2}+\varphi\left(Z_{2}\right)+\zeta+\left.\frac{1}{2} \beta_{2}\left(v, \psi\left(X_{2}\right)\right)\right|^{1 / 2} .
$$

From Lemma 3.14 it follows that

$$
\rho\left((\zeta, v),\left(g h g^{-1}\right)^{-1} \infty\right)=\left.\left|\frac{1}{4}\right| X_{2}\right|^{2}+\varphi\left(Z_{2}\right)+\zeta+\left.\frac{1}{2} \varphi\left(\beta_{2}\left(\psi^{-1}(v), X_{2}\right)\right)\right|^{1 / 2} .
$$

On the other side we find

$$
\begin{aligned}
\rho\left(g^{-1}(\zeta, v), h^{-1} \infty\right) & =\rho\left(\left(\varphi^{-1}(\zeta), \psi^{-1}(v)\right), h^{-1} \infty\right) \\
& =\left.\left|\frac{1}{4}\right| X_{2}\right|^{2}+Z_{2}+\varphi^{-1}(\zeta)+\left.\frac{1}{2} \beta_{2}\left(\psi^{-1}(v), X_{2}\right)\right|^{1 / 2} \\
& =\left.\left|\frac{1}{4}\right| X_{2}\right|^{2}+\varphi\left(Z_{2}\right)+\zeta+\left.\frac{1}{2} \varphi\left(\beta_{2}\left(\psi^{-1}(v), X_{2}\right)\right)\right|^{1 / 2} \\
& =\rho\left((\zeta, v),\left(g h g^{-1}\right)^{-1} \infty\right)
\end{aligned}
$$

From this it follows that $g I(h)=I\left(g h g^{-1}\right), g$ ext $I(h)=\operatorname{ext} I\left(g h g^{-1}\right)$, and $g$ int $I(h)=$ int $I\left(g h g^{-1}\right)$.

An immediate consequence of Proposition 3.15 is the following corollary.

Corollary 3.16 Let $\Gamma$ be a subgroup of $G$ and $g \in \Gamma_{\infty}$. Then

$$
g \bigcap_{h \in \Gamma \backslash \Gamma_{\infty}} \operatorname{ext} I(h)=\bigcap_{h \in \Gamma \backslash \Gamma_{\infty}} \operatorname{ext} I(h)
$$

and

$$
g \bigcup_{h \in \Gamma \backslash \Gamma_{\infty}} \operatorname{int} I(h)=\bigcup_{h \in \Gamma \backslash \Gamma_{\infty}} \operatorname{int} I(h) .
$$


Lemma 3.17 Let $z \in D$ and $g \in G \backslash G_{\infty}$. Then

$$
\operatorname{ht}(z)=\left(\frac{\rho\left(z, g^{-1} \infty\right)}{R(g)}\right)^{4} \operatorname{ht}(g z) .
$$

Proof Let $z=(\zeta, v)$ and $g=n_{1} \sigma m a_{t} n_{2}$ with $n_{j}=\left(1, Z_{j}, X_{j}\right)$ and $m=(\varphi, \psi)$. We first evaluate $\mathrm{ht}(g z)$. Set

$$
x:=\left[\varphi\left(\frac{1}{4}\left|X_{2}\right|^{2}+Z_{2}+\zeta+\frac{1}{2} \beta_{2}\left(v, X_{2}\right)\right)\right]^{-1} .
$$

Using (3.1) we find

$$
\begin{aligned}
\operatorname{ht}(g z)= & \operatorname{Re}\left(\frac{1}{4}\left|X_{1}\right|^{2}+Z_{1}+x t^{-1}-\frac{1}{2} t^{-1} \beta_{2}\left(x \psi\left(X_{2}+v\right), X_{1}\right)\right) \\
& -\frac{1}{4}\left|X_{1}-x t^{-1 / 2} \psi\left(X_{2}+v\right)\right|^{2} \\
= & \frac{1}{4}\left|X_{1}\right|^{2}+t^{-1} \operatorname{Re}(x)-\frac{1}{2} t^{-1 / 2}\left\langle x \psi\left(X_{2}+v\right), X_{1}\right\rangle \\
& -\frac{1}{4}\left|X_{1}-x t^{-1 / 2} \psi\left(X_{2}+v\right)\right|^{2} \\
= & t^{-1} \operatorname{Re}(x)-\frac{1}{4} t^{-1}|x|^{2} \cdot\left|X_{2}+v\right|^{2} \\
= & t^{-1}|x|^{2}\left[\operatorname{Re}\left(|x|^{-2} x\right)-\frac{1}{4}\left|X_{2}+v\right|^{2}\right] .
\end{aligned}
$$

Since $\operatorname{Re} \varphi(\eta)=\operatorname{Re} \eta$ for each $\eta \in C$, it follows that

$$
\begin{aligned}
\operatorname{Re}\left(|x|^{-2} x\right) & =\operatorname{Re}\left(\bar{x}^{-1}\right)=\operatorname{Re}\left(x^{-1}\right) \\
& =\operatorname{Re}\left(\frac{1}{4}\left|X_{2}\right|^{2}+Z_{2}+\zeta+\frac{1}{2} \beta_{2}\left(v, X_{2}\right)\right) \\
& =\frac{1}{4}\left|X_{2}\right|^{2}+\operatorname{Re} \zeta+\frac{1}{2}\left\langle v, X_{2}\right\rangle \\
& =\frac{1}{4}\left|X_{2}+v\right|^{2}+\operatorname{Re} \zeta-\frac{1}{4}|v|^{2} .
\end{aligned}
$$

Thus,

$$
\operatorname{ht}(g z)=t^{-1}|x|^{2}\left[\operatorname{Re} \zeta-\frac{1}{4}|v|^{2}\right]=t^{-1}|x|^{2} \operatorname{ht}(z) .
$$

Lemma 3.10 shows that

$$
\rho\left((\zeta, v), g^{-1} \infty\right)=\left.\left|\frac{1}{4}\right| X_{2}\right|^{2}+Z_{2}+\zeta+\left.\frac{1}{2} \beta_{2}\left(v, X_{2}\right)\right|^{1 / 2}=|x|^{-1 / 2} .
$$

Since $R(g)=t^{-1 / 4}$, we have

$$
\operatorname{ht}(z)=t|x|^{-2} \operatorname{ht}(g z)=\left(\frac{\rho\left(z, g^{-1} \infty\right)}{R(g)}\right)^{4} \operatorname{ht}(g z) .
$$




\subsection{Fundamental regions}

A subgroup $\Gamma$ of $G$ is said to be oftype $(O)$ if

$$
\bigcap_{g \in \Gamma \backslash \Gamma_{\infty}} \operatorname{ext} I(g)=D \backslash \overline{\bigcup_{g \in \Gamma \backslash \Gamma_{\infty}} \operatorname{int} I(g) .}
$$

For a subset $S$ of $G$ let $\langle S\rangle$ denote the subgroup of $G$ generated by $S$. Then $S$ is said to be of of type $(F)$, if for each $z \in D$ the maximum of the set

$$
\{\mathrm{ht}(g z) \mid g \in\langle S\rangle\}
$$

exists.

Theorem 3.18 Let $\Gamma$ be a subgroup of $G$ of type $(O)$ such that $\Gamma \backslash \Gamma_{\infty}$ is of type $(F)$. Suppose that $\mathcal{F}_{\infty}$ is a fundamental region for $\Gamma_{\infty}$ in D satisfying

$$
\overline{\mathcal{F}}_{\infty} \cap \bigcap_{g \in \Gamma \backslash \Gamma_{\infty}} \overline{\operatorname{ext} I(g)}=\overline{\mathcal{F}_{\infty} \cap \bigcap_{g \in \Gamma \backslash \Gamma_{\infty}} \operatorname{ext} I(g)} .
$$

Then

$$
\mathcal{F}:=\mathcal{F}_{\infty} \cap \bigcap_{g \in \Gamma \backslash \Gamma_{\infty}} \operatorname{ext} I(g)
$$

is a fundamental region for $\Gamma$ in $D$.

Proof Since $\Gamma$ is of type (O), the set $\bigcap\left\{\operatorname{ext} I(g) \mid g \in \Gamma \backslash \Gamma_{\infty}\right\}$ is open. Then $\mathcal{F}_{\infty}$ being open as a fundamental region for $\Gamma_{\infty}$ in $D$ yields that $\mathcal{F}$ is open.

Now let $z \in \mathcal{F}$ and $g \in \Gamma \backslash\{$ id $\}$. If $g \in \Gamma_{\infty}$, then $g z \notin \mathcal{F}_{\infty}$ since the $\Gamma_{\infty}$-translates of $\mathcal{F}_{\infty}$ are pairwise disjoint. If $g \in \Gamma \backslash \Gamma_{\infty}$, then $z \in$ ext $I(g)$. Lemma 3.13 states that $g z \in \operatorname{int} I\left(g^{-1}\right)$, and hence $g z \notin \operatorname{ext} I\left(g^{-1}\right)$. Thus, in each case, $g z \notin \mathcal{F}$. This shows that the $\Gamma$-translates of $\mathcal{F}$ are pairwise disjoint.

It remains to prove that $D \subseteq \Gamma \cdot \overline{\mathcal{F}}$. To that end let $z \in D$ and set

$$
A:=\bigcap\left\{\overline{\operatorname{ext} I(g)} \mid g \in \Gamma \backslash \Gamma_{\infty}\right\} .
$$

Since $\Gamma \backslash \Gamma_{\infty}$ is of type (F), the set $\left\langle\Gamma \backslash \Gamma_{\infty}\right\rangle z$ contains an element of maximal height, say $w$. We claim that $w \in A$. Seeking a contradiction assume that $w \notin A$. Proposition 3.12 yields the existence of $h \in \Gamma \backslash \Gamma_{\infty}$ with $w \in$ int $I(h)$. Then, by definition,

$$
\rho\left(w, h^{-1} \infty\right)<R(h) .
$$

This and Lemma 3.17 show that ht $(h w)>\operatorname{ht}(w)$, which is a contradiction to the choice of $w$. Thus, $w \in A$.

Since $\Gamma_{\infty} \overline{\mathcal{F}_{\infty}}=D$, there is $k \in \Gamma_{\infty}$ such that $k w \in \overline{\mathcal{F}}_{\infty}$. Corollary 3.16 implies that $k w \in A$. Finally,

$$
k w \in \overline{\mathcal{F}}_{\infty} \cap A=\overline{\mathcal{F}_{\infty} \cap \bigcap_{g \in \Gamma \backslash \Gamma_{\infty}} \operatorname{ext} I(g)}=\overline{\mathcal{F}} .
$$

This completes the proof. 
Remark 3.19 Let $\Gamma$ be a subgroup of $G$ and suppose that the set

$$
\left\{\text { int } I(g) \mid g \in \Gamma \backslash \Gamma_{\infty}\right\}
$$

of interiors of all isometric spheres is locally finite. Then

$$
\overline{\bigcup_{g \in \Gamma \backslash \Gamma_{\infty}} \operatorname{int} I(g)}=\bigcup_{g \in \Gamma \backslash \Gamma_{\infty}} \overline{\operatorname{int} I(g)} .
$$

by [20, Hilfssatz 7.14]. Lemma 3.11(iii) implies that

$$
\complement\left(\overline{\bigcup_{g \in \Gamma \backslash \Gamma_{\infty}} \operatorname{int} I(g)}\right)=\complement\left(\bigcup_{g \in \Gamma \backslash \Gamma_{\infty}} \overline{\operatorname{int} I(g)}\right)=\bigcap_{g \in \Gamma \backslash \Gamma_{\infty}} \operatorname{Cint~I(g)}=\bigcap_{g \in \Gamma \backslash \Gamma_{\infty}} \operatorname{ext} I(g) \text {. }
$$

Hence, if the set of interiors of isometric spheres is locally finite, then $\Gamma$ is of type $(O)$.

A special case of Theorem 3.18 is the following corollary.

Corollary 3.20 Let $\Gamma$ be a subgroup of $G$ of type $(O)$ and $\Gamma_{\infty}=\{\mathrm{id}\}$. Further suppose that $\Gamma \backslash \Gamma_{\infty}$ is of type $(F)$. Then

$$
\mathcal{F}:=\bigcap_{g \in \Gamma \backslash \Gamma_{\infty}} \operatorname{ext} I(g)
$$

is a fundamental region for $\Gamma$ in $D$.

The following proposition serves to show that in many situations the fundamental region in Theorem 3.18 is actually a fundamental domain (see Corollary 3.23 below).

Proposition 3.21 Suppose that $\mathfrak{v}=\{0\}$. Then the set ext $I(g)$ is geodesically convex for each $g \in G \backslash G_{\infty}$.

Proof Let $z_{1}, z_{2} \in \operatorname{ext} I(g), z_{1} \neq z_{2}$. Then there exist $s_{2}>s_{1}>0$ and $h \in G$ such that $z_{1}=h a_{s_{1}} \cdot o_{D}$ and $z_{2}=h a_{s_{2}} \cdot o_{D}$. W.l.o.g. we may assume that $h$ is either of the form $h=n \in N$ (if $h \in G_{\infty}$ ) or $h=n^{\prime} \sigma n \in N \sigma N$ (if $h \in G \backslash G_{\infty}$ ). The geodesic segment connecting $z_{1}$ and $z_{2}$ is given by

$$
c:=\left\{h a_{r} \cdot o_{D} \mid s_{1} \leq r \leq s_{2}\right\} .
$$

In the following we will show that $c \subseteq$ ext $I(g)$ by examining separately the two cases for $h$.

Suppose that $g=n_{1} \sigma m a_{t} n_{2}$ with $n_{1}, n_{2}=\left(1, Z_{s}\right) \in N, a_{t} \in A$ and $m \in M$ and $r \in\left[s_{1}, s_{2}\right]$. At first let $h=n=(1, Z) \in N$. Then

$$
\begin{aligned}
\rho\left(n a_{r} \cdot o_{D}, g^{-1} \infty\right) & =\rho\left((r, Z), g^{-1} \infty\right)=\left|Z_{s}+Z+r\right|^{1 / 2}=\left(\left|Z_{s}+Z\right|^{2}+r^{2}\right)^{1 / 4} \\
& \geq\left(\left|Z_{s}+Z\right|^{2}+s_{1}^{2}\right)^{1 / 4}=\rho\left(n a_{s_{1}} \cdot o_{D}, g^{-1} \infty\right)>R(g) .
\end{aligned}
$$

Hence $h a_{r} \cdot o_{D} \in \operatorname{ext} I(g)$.

Now let $h=n^{\prime} \sigma n \in N \sigma N$ with $n^{\prime}=\left(1, Z^{\prime}\right)$ and $n=(1, Z)$. A straightforward calculation shows that

$$
\rho\left(h a_{r} \cdot o_{D}, g^{-1} \infty\right)>R(g)=t^{-1 / 4}
$$

is equivalent to

$$
0<a r^{4}+b r^{2}+c
$$


where $Z_{s}^{\prime}:=Z_{s}+Z^{\prime}$ and

$$
\begin{aligned}
a & :=\left|Z_{s}^{\prime}\right|^{2}-t^{-1} \\
b & :=1+2|Z|^{2}\left|Z_{s}^{\prime}\right|^{2}-2\left\langle Z, Z_{s}^{\prime}\right\rangle-2|Z|^{2} t^{-1} \\
c & :=|Z|^{4}\left|Z_{s}^{\prime}\right|^{2}-2|Z|^{2}\left\langle Z, Z_{s}^{\prime}\right\rangle+|Z|^{2}-|Z|^{4} t^{-1} .
\end{aligned}
$$

Solving (3.2) separately for $a=0, a>0$ and $a<0$ yields that it is satisfied for $r$. Therefore, $h a_{r} \cdot o_{D} \in \operatorname{ext} I(g)$. This completes the proof.

Remark 3.22 Using the classification in [15] of $J^{2} C$-module structures and the statement [5, Proposition 2.5.1] on the (non-)existence of totally geodesic submanifolds of codimension one in rank one Riemannian symmetric spaces we see that Proposition 3.21 in general becomes false if $\mathfrak{v} \neq\{0\}$.

Theorem 3.18 and Proposition 3.21 immediately imply the following statement.

Corollary 3.23 Let $\mathfrak{v}=\{0\}$ and let $\Gamma$ be a subgroup of $G$ of type $(O)$ such that $\Gamma \backslash \Gamma_{\infty}$ is of type $(F)$. Suppose that $\mathcal{F}_{\infty}$ is a convex fundamental domain for $\Gamma_{\infty}$ in $D$ satisfying

$$
\overline{\mathcal{F}}_{\infty} \cap \bigcap_{g \in \Gamma \backslash \Gamma_{\infty}} \overline{\operatorname{ext} I(g)}=\overline{\mathcal{F}_{\infty} \cap \bigcap_{g \in \Gamma \backslash \Gamma_{\infty}} \operatorname{ext} I(g) .}
$$

Then

$$
\mathcal{F}:=\mathcal{F}_{\infty} \cap \bigcap_{g \in \Gamma \backslash \Gamma_{\infty}} \operatorname{ext} I(g)
$$

is a convex fundamental domain for $\Gamma$ in $D$.

\section{Projective models}

The purpose of this section is to show that the existing definitions of isometric spheres and results concerning the existence of isometric fundamental regions in literature are essentially covered by the definitions and results in Sect. 3. The reason for the reservation towards a confirmation to cover all existing definitions and results is twofold: On the one hand the author cannot guarantee to be aware of all existing results. On the other hand, at least for the real hyperbolic plane, the literature contains non-equivalent definitions of isometric spheres. Moreover, the existence results of isometric fundamental regions by Ford are proved for a weaker notion of fundamental region than the one used here. Section 4.7 contains a detailed discussion of the latter issues.

Let $(C, V, J)$ be a $J^{2} C$-module structure. In Sect. 4.1 we introduce the structure of division algebras on $C$ following [15] and [16]. For $C$ being an associative division algebra, we redo, in Sects. 4.3 and 4.4, the classical projective construction of hyperbolic spaces in terms of the $J^{2} C$-module structure. A long part of Sect. 4.4 is devoted to a detailed study of the relation between the isometry group $G$ of the symmetric space and the natural "matrix" group on the projective space. This investigation will show that the matrix group is isomorphic to a certain subgroup $G^{\text {res }}$ of $G$. In Sect. 4.5, we use these results to provide a characterization of the isometric sphere of $g \in G^{\text {res }}$ via a cocycle. In Sect. 4.6, we prove that a special class of subgroups $\Gamma$ of $G^{\text {res }}$ are of type $(\mathrm{O})$ with $\Gamma \backslash \Gamma_{\infty}$ being of type $(\mathrm{F})$ and use Theorem 3.18 to show the existence of an isometric fundamental domain for $\Gamma$. Finally, in Sect. 4.7, we bring together these investigations for a comparison with the existing literature. 
4.1 Division algebras induced by $J^{2} C$-module structures

Let $(C, V, J)$ be a $J^{2} C$-module structure. Suppose that $V \neq\{0\}$ and fix an element $v \in V \backslash\{0\}$. From (M3) and (M2) it follows that for each pair $(\zeta, \eta) \in C \times C$ there exists a unique element $\tau \in C$ such that

$$
J(\zeta, J(\eta, v))=J(\tau, v) .
$$

Hence, each choice $v \in V \backslash\{0\}$ equips $C$ with a multiplication ${ }_{v}: C \times C \rightarrow C$ via

$$
\zeta \cdot v \eta:=\tau \quad \Leftrightarrow \quad J(\zeta, J(\eta, v))=J(\tau, v) .
$$

Equation (2.9) shows that the inverse of $\zeta \in C \backslash\{0\}$ is $\zeta^{-1}=|\zeta|^{-2} \bar{\zeta}$, independent of the choice of $v \in V \backslash\{0\}$. The following properties of $(C, \cdot v)$ are shown in [15].

Proposition 4.1 (Proposition 1.1 and Corollary 1.5 in [15])

(i) For each $v \in V \backslash\{0\}$, the Euclidean vector space $C$ with the multiplication ${ }_{v} v$ is a normed, not necessarily associative, division algebra.

(ii) The multiplication $\cdot v$ on $C$ is independent of the choice of $v \in V \backslash\{0\}$ if and only if $(C, \cdot v)$ is associative for one (and hence for all) $v$.

We call the $J^{2} C$-module structure $(C, V, J)$ associative if $\left(C,{ }_{v}\right)$ is associative for some (and hence each) $v \in V \backslash\{0\}$ and otherwise non-associative. If $(C, \cdot v)$ is associative, we omit the subscript $v$ of the multiplication $\cdot v$.

Remark 4.2 Only associative $J^{2} C$-module structures are modules in the sense of [4].

Remark 4.3 Suppose that $(C, V, J)$ is an associative $J^{2} C$-module structure. The classification in [15] (or [16, Sect. 4]) shows that $C$ is real or complex or quaternionic numbers.

\section{2 $C$-sesquilinear hermitian forms}

From now on let $(C, V, J)$ be an associative $J^{2} C$-module structure. Suppose that $M$ is a (left) $C$-module. A map $\Phi: M \times M \rightarrow C$ is said to be a $C$-sesquilinear hermitian form if $\Phi$ is $C$-hermitian and $C$-linear in the first variable, that is, if the following properties are satisfied:

(SH1) $\Phi\left(\zeta_{1} x_{1}+\zeta_{2} x_{2}, y\right)=\zeta_{1} \Phi\left(x_{1}, y\right)+\zeta_{2} \Phi\left(x_{2}, y\right)$ for all $\zeta_{1}, \zeta_{2} \in C$ and $x, y \in M$, (SH2) $\Phi(x, y)=\overline{\Phi(y, x)}$ for all $x, y \in M$.

A $C$-sesquilinear hermitian form $\Phi$ is called non-degenerate if $\Phi\left(m_{1}, \cdot\right)=0$ implies that $m_{1}=0$. It is called indefinite if there exist $m_{1}, m_{2} \in M$ such that $\Phi\left(m_{1}, m_{1}\right)<0$ and $\Phi\left(m_{2}, m_{2}\right)>0$.

Proposition 4.4 The map $\beta_{1}: C \times C \rightarrow C, \beta_{1}(x, y):=x \bar{y}$, is $C$-sesquilinear hermitian. Further $\operatorname{Re} \beta_{1}(x, y)=\langle x, y\rangle$ for all $x, y \in C$.

Proof Obviously, $\beta_{1}$ is $\mathbb{R}$-bilinear. For each $y \in C$, the $C$-linearity of $\beta_{1}(\cdot, y)$ is exactly the left-sided distribution law of the division algebra $C$. To show (SH2) let $x, y \in C$. From (2.7) it follows that

$$
J_{\bar{y} \bar{x}}=J_{y \bar{x}}^{*}=J_{\bar{x}}^{*} J_{y}^{*}=J_{x} J_{\bar{y}}=J_{x \bar{y}} .
$$


Therefore,

$$
\beta_{1}(x, y)=x \bar{y}=\overline{y \bar{x}}=\overline{\beta_{1}(y, x)} .
$$

Hence $\beta_{1}$ is $C$-sesquilinear hermitian. Finally, polarization of $\beta_{1}(x, x)=|x|^{2}$ (see (2.8)) over $\mathbb{R}$ implies the remaining statement.

Remark 4.5 An immediate consequence of Proposition 4.4 is that conjugation and multiplication in $C$ anticommute, i.e., $\bar{x} \bar{y}=\overline{y x}$ for all $x, y \in C$. In particular, for a $C$-sesquilinear hermitian form $\Phi$ on the $C$-module $M$ we have

$$
\Phi\left(m_{1}, \zeta m_{2}\right)=\Phi\left(m_{1}, m_{2}\right) \bar{\zeta}
$$

for all $m_{1}, m_{2} \in M$ and all $\zeta \in C$. Moreover, we have $\Phi(m, m)=\overline{\Phi(m, m)}$ for each $m \in M$ and therefore, $\Phi(m, m) \in \mathbb{R}$.

Lemma 4.6 For all $\zeta, \eta, \xi \in C$ we have $\langle\zeta, \xi \eta\rangle=\langle\bar{\xi} \zeta, \eta\rangle$.

Proof Let $v \in V \backslash\{0\}$. Using (2.6) and (2.7) we find

$$
2\langle\zeta, \xi \eta\rangle|v|^{2}=2\langle\zeta v, \xi \eta v\rangle=2\langle\bar{\xi} \zeta v, \eta v\rangle=2\langle\bar{\xi} \zeta, \eta\rangle|v|^{2},
$$

hence $\langle\zeta, \xi \eta\rangle=\langle\bar{\xi} \zeta, \eta\rangle$.

Proposition 4.7 The map $\beta_{2}: V \times V \rightarrow C$ is $C$-sesquilinear hermitian.

Proof Because of Proposition 2.11 it remains to show that $\beta_{2}$ is $C$-linear in the first variable. Let $v, u \in V$ and $\zeta \in C$. Lemma 4.6 and (2.7) yield that for all $\eta \in C$ we have

$$
\left\langle\zeta \beta_{2}(v, u), \eta\right\rangle=\left\langle\beta_{2}(v, u), \bar{\zeta} \eta\right\rangle=\langle\bar{\zeta} \eta u, v\rangle=\langle\eta u, \zeta v\rangle=\left\langle\beta_{2}(\zeta v, u), \eta\right\rangle .
$$

Hence $\zeta \beta_{2}(v, u)=\beta_{2}(\zeta v, u)$.

A finite sequence $\left(v_{1}, \ldots, v_{n}\right)$ in $V$ is called an orthonormal $C$-basis of $V$ if

(CON1) $\left|v_{j}\right|=1$ for each $j \in\{1, \ldots, n\}$,

(CON2) for each pair $(i, j) \in\{1, \ldots, n\}^{2}, i \neq j$, the sets $C v_{i}$ and $C v_{j}$ are orthogonal,

(CON3) if we use for each $j \in\{1, \ldots, n\}$ the bijection $C \rightarrow C v_{j}, \zeta \mapsto \zeta v_{j}$, to equip $C v_{j}$ with the structure of a Euclidean vector space and a $C$-module, then $V$ is isomorphic as $C$-module and Euclidean vector space to the direct sum $\bigoplus_{j=1}^{n} C v_{j}$ of the Euclidean spaces and $C$-modules $C v_{j}, j=1, \ldots, n$.

The following lemma yields that $V$ is a free $C$-module.

Lemma 4.8 There is an orthonormal C-basis of $V$.

Proof Clearly, one finds a sequence $\left(v_{1}, \ldots, v_{n}\right)$ in $V$ which satisfies (CON1) and (CON2) such that $V$ is isomorphic as Euclidean vector space to $\bigoplus_{j=1}^{n} C v_{j}$. From Propositions 4.7 and 2.11 it follows that

$$
\psi:\left\{\begin{aligned}
V & \rightarrow \bigoplus_{j=1}^{n} C v_{j} \\
v & \mapsto \sum_{j=1}^{n} \beta_{2}\left(v, v_{j}\right) v_{j}
\end{aligned}\right.
$$

is an isomorphism (of Euclidean vector spaces) from $V$ to $\bigoplus_{j=1}^{n} C v_{j}$. Let $v \in V, \eta \in C$ and suppose that $v$ is isomorphic to $\left(\zeta_{1} v_{1}, \ldots, \zeta_{n} v_{n}\right) \in \bigoplus_{j=1}^{n} C v_{j}$. Then

$$
\psi(\eta v)=\sum_{j=1}^{n} \beta_{2}\left(\eta v, v_{j}\right) v_{j}=\sum_{j=1}^{n}\left(\eta \beta_{2}\left(v, v_{j}\right)\right) v_{j}=\eta\left(\sum_{j=1}^{n} \beta_{2}\left(v, v_{j}\right) v_{j}\right)=\eta \psi(v) .
$$

This shows that $\psi$ is indeed an isomorphism of $C$-modules. 


\subsection{The $C$-projective space $P_{C}(E)$}

Let $(C, V, J)$ be an associative $J^{2} C$-module structure. Further let $W:=C \oplus V$ be the Euclidean direct sum of $C$ and $V$, and $E:=C \oplus W=C \oplus C \oplus V$ be that of $C$ and $W$. We define a $C$-multiplication on $E$ by

$$
\therefore \begin{cases}C \times E & \rightarrow E \\ (\tau,(\zeta, \eta, v)) & \mapsto(\tau \zeta, \tau \eta, \tau v)\end{cases}
$$

Because $(C, V, J)$ is associative and hence $\sigma(\tau v)=(\sigma \tau) v$ for each $v \in V$ and all $\sigma, \tau \in C$ (or equivalently, since the definition of the product in $C$ does not depend on the choice of $v \in V \backslash\{0\}$, see Proposition 4.1), $E$ becomes a $C$-module. Since $V$ is a free $C$-module by Lemma 4.8, also $E$ is a free $C$-module.

Two elements $z_{1}, z_{2}$ of $E \backslash\{0\}$ are called equivalent $\left(z_{1} \sim z_{2}\right)$ if there is $\tau \in C$ such that $\tau z_{1}=z_{2}$. Note that $\tau$ is actually in $C \backslash\{0\}$. Then $E$ being a $C$-module implies that $\sim$ is indeed an equivalence relation on $E \backslash\{0\}$. The $C$-projective space $P_{C}(E)$ of $E$ is defined as the set of equivalence classes of $\sim$,

$$
P_{C}(E):=(E \backslash\{0\}) / \sim,
$$

endowed with the induced topology and the differential structure generated by the following (standard) charts: Let $\left\{v_{1}, \ldots, v_{n-1}\right\}$ be an orthonormal $C$-basis of $V$. Then $E$ is isomorphic to $C^{n+1}$ both as Euclidean vector space and $C$-module. For each $j=1, \ldots, n+1$ the set

$$
U_{j}:=\left\{\left[\left(\zeta_{1}, \ldots, \zeta_{n+1}\right)\right] \in P_{C}(E) \mid \zeta_{j} \neq 0\right\}
$$

is open, and the maps $\varphi_{j}: U_{j} \rightarrow C^{n}$

$$
\varphi_{j}\left(\left[\left(\zeta_{1}, \ldots, \zeta_{n+1}\right)\right]\right):=\zeta_{j}^{-1}\left(\zeta_{1}, \ldots, \widehat{\zeta}_{j}, \ldots, \zeta_{n+1}\right)
$$

are pairwise compatible in the sense that they are real differentiable (they are not $C$-differentiable unless $C$ is commutative). Here, $\widehat{\zeta}_{j}$ means that $\zeta_{j}$ is omitted, hence $\left(\zeta_{1}, \ldots, \widehat{\zeta}_{j}, \ldots\right.$, $\left.z_{n+1}\right)=\left(\zeta_{1}, \ldots, \zeta_{j-1}, \zeta_{j+1}, \ldots, \zeta_{n+1}\right) \in C^{n}$. Obviously, the differential structure is independent of the choice of the orthonormal $C$-basis of $V$, and $P_{C}(E)$ is a real smooth manifold of dimension $n \cdot \operatorname{dim}_{\mathbb{R}} C$.

Let $\Phi$ be a $C$-sesquilinear hermitian form on $E$. Recall from Remark 4.5 that $\Phi(z, z) \in \mathbb{R}$ for each $z \in E$. Suppose that $q$ is its associated quadratic form, that is

$$
q:\left\{\begin{aligned}
E & \rightarrow \mathbb{R} \\
z & \mapsto \Phi(z, z) .
\end{aligned}\right.
$$

Then we define the following sets:

$$
\begin{array}{ll}
E_{-}(\Phi):=q^{-1}((-\infty, 0)) & \text { the set of } \Phi \text {-negative vectors, } \\
E_{0}(\Phi):=q^{-1}(0) \backslash\{0\} & \text { the set of } \Phi \text {-zero vectors, and } \\
E_{+}(\Phi):=q^{-1}((0, \infty)) & \text { the set of } \Phi \text {-positive vectors. }
\end{array}
$$

For each $\tau \in C$ and $z \in E \backslash\{0\}$ we have

$$
q(\tau z)=\Phi(\tau z, \tau z)=\tau \Phi(z, z) \bar{\tau}=\Phi(z, z) \tau \bar{\tau}=q(z)|\tau|^{2} .
$$

Lemma 4.9 Let $\Phi$ be a $C$-sesquilinear hermitian form on $E$. The set $P_{C}(E)$ equals the disjoint union $P_{C}\left(E_{-}(\Phi)\right) \cup P_{C}\left(E_{0}(\Phi)\right) \cup P_{C}\left(E_{+}(\Phi)\right)$. 
Proof Clearly, $P_{C}(E)=P_{C}\left(E_{-}(\Phi)\right) \cup P_{C}\left(E_{0}(\Phi)\right) \cup P_{C}\left(E_{+}(\Phi)\right)$. Hence it remains to prove that this union is disjoint. Suppose that $q$ denotes the quadratic form associated to $\Phi$. If we assume for contradiction that

$$
P_{C}\left(E_{-}(\Phi)\right) \cap P_{C}\left(E_{0}(\Phi)\right) \neq \emptyset,
$$

then there are equivalent $z_{1}, z_{2} \in E \backslash\{0\}$ such that $q\left(z_{1}\right)<0$ and $q\left(z_{2}\right)=0$. But then there is $\tau \in C \backslash\{0\}$ such that

$$
0=q\left(z_{2}\right)=q\left(\tau z_{1}\right)=q\left(z_{1}\right)|\tau|^{2}<0,
$$

which is a contraction. Hence $P_{C}\left(E_{-}(\Phi)\right) \cap P_{C}\left(E_{0}(\Phi)\right)=\emptyset$. Analogously we see that $P_{C}\left(E_{-}(\Phi)\right) \cap P_{C}\left(E_{+}(\Phi)\right)=\emptyset$ and $P_{C}\left(E_{+}(\Phi)\right) \cap P_{C}\left(E_{0}(\Phi)\right)=\emptyset$.

Let $\pi: E \backslash\{0\} \rightarrow P_{C}(E)$ denote the projection on the equivalence classes. Since $C \backslash\{0\}$ acts homeomorphically on $E \backslash\{0\}$, the projection $\pi$ is open. Further $\pi$ is continuous by the definition of the topology on $P_{C}(E)$.

Proposition 4.10 Let $\Phi: E \times E \rightarrow C$ be a $C$-sesquilinear hermitian form, which is indefinite and non-degenerate. Then

$$
\partial P_{C}\left(E_{-}(\Phi)\right)=P_{C}\left(E_{0}(\Phi)\right) .
$$

Proof Let $U \subseteq P_{C}\left(E_{-}(\Phi)\right)$. All complements, closures, interiors, and boundaries of subsets of $E \backslash\{0\}$ are taken in $E \backslash\{0\}$. At first we show that

$$
\complement \pi\left(\complement \partial \pi^{-1}(U)\right)=\partial U .
$$

To that end let $M:=\pi^{-1}(U)$. Then $\complement M=\pi^{-1}(\complement U)$. From $\pi$ being open and continuous it follows that $(\complement U)^{\circ}=\pi\left((\complement M)^{\circ}\right)$. This yields

$$
\pi(\complement \bar{M})=\pi\left((\complement M)^{\circ}\right)=(\complement U)^{\circ}=\complement \bar{U},
$$

hence $\complement \pi(\complement \bar{M})=\bar{U}$. Again from $\pi$ being open and continuous we get $U^{\circ}=\pi\left(M^{\circ}\right)$. Therefore,

$$
\begin{aligned}
\complement \pi\left(\complement \partial \pi^{-1}(U)\right) & =\complement \pi(\complement \partial M) \\
& =\complement \pi\left(\complement\left(\bar{M} \cap \complement M^{\circ}\right)\right) \\
& =\complement\left(\pi(\complement \bar{M}) \cup \pi\left(M^{\circ}\right)\right) \\
& =\complement \pi(\complement \bar{M}) \cap \complement \pi\left(M^{\circ}\right) \\
& =\bar{U} \cap \complement U^{\circ}=\partial U .
\end{aligned}
$$

Let $q$ denote the quadratic form associated to $\Phi$. Then $q$ is smooth. Since $\Phi$ is non-degenerate, 0 is a regular value for $\left.q\right|_{E \backslash\{0\}}$. Hence $E_{0}(\Phi)=q^{-1}(0) \backslash\{0\}$ is the boundary of the bounded submanifold $q^{-1}((-\infty, 0]) \backslash\{0\}$ and therefore also of $E_{-}(\Phi)=q^{-1}((-\infty, 0))$. Then the statement follows from (4.2) and Lemma 4.9 with $U:=P_{C}\left(E_{-}(\Phi)\right)$.

\subsection{The $C$-hyperbolic spaces $P_{C}\left(E_{-}\left(\Psi_{1}\right)\right)$ and $P_{C}\left(E_{-}\left(\Psi_{2}\right)\right)$}

Let $(C, V, J)$ be an associative $J^{2} C$-module structure. We define two specific non-degenerate, indefinite $C$-sesquilinear hermitian forms $\Psi_{1}$ and $\Psi_{2}$ and consider the manifolds $P_{C}\left(E_{-}\left(\Psi_{j}\right)\right)$ defined in Sect. 4.3. For each space we choose a set of representatives and a Riemannian metric on it such that $P_{C}\left(E_{-}\left(\Psi_{1}\right)\right)$ is essentially the ball model from Sect. 2.5 
and $P_{C}\left(E_{-}\left(\Psi_{2}\right)\right)$ becomes the Siegel domain model for hyperbolic space, which is essentially the model $D$ from Sect. 2.4 .

We denote by $\mathrm{GL}_{C}(E)$ the group of all $C$-linear invertible maps $E \rightarrow E$. Set

$$
U\left(\Psi_{j}, C\right):=\left\{g \in \operatorname{GL}_{C}(E) \mid \forall z_{1}, z_{2} \in E: \Psi_{j}\left(g z_{1}, g z_{2}\right)=\Psi_{j}\left(z_{1}, z_{2}\right)\right\},
$$

and let $Z\left(\Psi_{j}, C\right)$ denote the center of $U\left(\Psi_{j}, C\right)$. Recall that $G$ denotes the full isometry group of $B$, respectively $D$. In this section, we establish a natural and explicit isomorphism between the quotient group $\mathrm{PU}\left(\Psi_{j}, C\right):=U\left(\Psi_{j}, C\right) / Z\left(\Psi_{j}, C\right)$ and a subgroup $G^{\text {res }}$ of $G$. Moreover, we explicitly characterize $G^{\text {res }}$. In Sect. 4.5 , this isomorphism is used to show that the definition of isometric spheres in literature is subsumed by our definition.

Let $\beta_{3}: W \times W \rightarrow C$ be the sum of $\beta_{1}$ and $\beta_{2}$, that is,

$$
\beta_{3}\left(\left(\eta_{1}, v_{1}\right),\left(\eta_{2}, v_{2}\right)\right):=\beta_{1}\left(\eta_{1}, \eta_{2}\right)+\beta_{2}\left(v_{1}, v_{2}\right) .
$$

We define the maps $\Psi_{j}: E \times E \rightarrow C(j=1,2)$ by

$$
\begin{aligned}
\Psi_{1}\left(\left(\zeta_{1}, \eta_{1}, v_{1}\right),\left(\zeta_{2}, \eta_{2}, v_{2}\right)\right) & :=-\beta_{1}\left(\zeta_{1}, \zeta_{2}\right)+\beta_{1}\left(\eta_{1}, \eta_{2}\right)+\beta_{2}\left(v_{1}, v_{2}\right) \\
& =-\beta_{1}\left(\zeta_{1}, \zeta_{2}\right)+\beta_{3}\left(\left(\eta_{1}, v_{1}\right),\left(\eta_{2}, v_{2}\right)\right)
\end{aligned}
$$

and

$$
\Psi_{2}\left(\left(\zeta_{1}, \eta_{1}, v_{1}\right),\left(\zeta_{2}, \eta_{2}, v_{2}\right)\right):=-\beta_{1}\left(\zeta_{1}, \eta_{2}\right)-\beta_{1}\left(\eta_{1}, \zeta_{2}\right)+\beta_{2}\left(v_{1}, v_{2}\right)
$$

Let $q_{j}$ denote the associated quadratic forms. For all $(\zeta, w)=(\zeta, \eta, v) \in E$ we have

$$
q_{1}((\zeta, w))=q_{1}((\zeta, \eta, v))=-|\zeta|^{2}+|\eta|^{2}+|v|^{2}=-|\zeta|^{2}+|w|^{2} .
$$

Employing Proposition 4.4, we see that

$$
q_{2}((\zeta, \eta, v))=-2 \operatorname{Re} \beta_{1}(\zeta, \eta)+|v|^{2}=-2\langle\zeta, \eta\rangle+|v|^{2} .
$$

Lemma 4.11 For $j=1,2$, the map $\Psi_{j}$ is a $C$-sesquilinear hermitian form on $E$ which is non-degenerate and indefinite.

Proof Propositions 4.4 and 4.7 imply that $\Psi_{j}$ is $C$-sesquilinear hermitian. Suppose that there is $(\zeta, \eta, v) \in E$ such that for all $(\sigma, \tau, u) \in E$ we have

$$
\Psi_{1}((\zeta, \eta, v),(\sigma, \tau, u))=0 .
$$

Propositions 4.4 and 2.11 show that

$$
0=\Psi_{1}((\zeta, \eta, v),(0, \eta, v))=|\eta|^{2}+|v|^{2} .
$$

Hence $(\eta, v)=(0,0)$. Further

$$
0=\Psi_{1}((\zeta, \eta, v),(\zeta, 0,0))=-|\zeta|^{2},
$$

and therefore $\zeta=0$. This shows that $\Psi_{1}$ is non-degenerate. Suppose now that there is $(\zeta, \eta, v) \in E$ such that for all $(\sigma, \tau, u) \in E$ we have

$$
\Psi_{2}((\zeta, \eta, v),(\sigma, \tau, u))=0 .
$$

Then

$$
0=\Psi_{2}((\zeta, \eta, v),(0,0, v))=|v|^{2}
$$


and therefore $v=0$. Moreover,

$$
0=\Psi_{2}((\zeta, \eta, v),(0, \zeta, 0))=-|\zeta|^{2} .
$$

Thus $\zeta=0$ and analogously $\eta=0$. Hence $\Psi_{2}$ is non-degenerate. Finally, for (any) $v \in V \backslash\{0\}$,

$$
\begin{aligned}
& q_{1}((1,0,0))=-1 \quad \text { and } \quad q_{1}((0,0, v))=|v|^{2}>0, \\
& q_{2}((1,1,0))=-2 \quad \text { and } \quad q_{2}((0,0, v))=|v|^{2}>0 .
\end{aligned}
$$

Therefore, $\Psi_{1}$ and $\Psi_{2}$ are indefinite.

\subsubsection{Set of representatives for $\overline{P_{C}\left(E_{-}\left(\Psi_{1}\right)\right)}$}

Proposition 4.10 shows that we have

$$
\overline{P_{C}\left(E_{-}\left(\Psi_{j}\right)\right)}=P_{C}\left(E_{-}\left(\Psi_{j}\right)\right) \cup P_{C}\left(E_{0}\left(\Psi_{j}\right)\right)
$$

for $j \in\{1,2\}$. If $z=(\zeta, w) \in E_{-}\left(\Psi_{1}\right) \cup E_{0}\left(\Psi_{1}\right)$, then $\zeta \neq 0$. Therefore, the element $[z] \in P_{C}\left(E_{-}\left(\Psi_{1}\right)\right) \cup P_{C}\left(E_{0}\left(\Psi_{1}\right)\right)$ is represented by $\left(1, \zeta^{-1} w\right)$, and this is the unique representative of the form $(1, *)$. If $z \in E_{-}\left(\Psi_{1}\right)$, then

$$
0>q_{1}\left(1, \zeta^{-1} w\right)=-1+\left|\zeta^{-1} w\right|^{2} .
$$

This shows that $1>\left|\zeta^{-1} w\right|^{2}$. Conversely, if $w \in W$ with $|w|^{2}<1$, then $[(1, w)]$ is an element of $P_{C}\left(E_{-}\left(\Psi_{1}\right)\right)$. Recall the unit ball $B=\{w \in W|| w \mid<1\}$ in $W$. Then $P_{C}\left(E_{-}\left(\Psi_{1}\right)\right)$ and $B$ are in bijection via the map

$$
[(\zeta, w)] \mapsto \zeta^{-1} w
$$

The same argument shows that $\partial P_{C}\left(E_{-}\left(\Psi_{1}\right)\right)=P_{C}\left(E_{0}\left(\Psi_{1}\right)\right)$ is bijective to $\partial B$ via this map. We define $\tau_{B}: \overline{P_{C}\left(E_{-}\left(\Psi_{1}\right)\right)} \rightarrow \bar{B}$ as

$$
\tau_{B}([(\zeta, w)]):=\zeta^{-1} w .
$$

Its inverse is

$$
\tau_{B}^{-1}(w)=[(1, w)] .
$$

In comparison with Sect. 4.3 , we see that $\overline{P_{C}\left(E_{-}\left(\Psi_{1}\right)\right)}$ is a subset of $U_{1}$ and $\tau_{B}$ a restriction of $\varphi_{1}$. Therefore, $\tau_{B}$ is a diffeomorphism between the manifolds $P_{C}\left(E_{-}\left(\Psi_{1}\right)\right)$ and $B$, and also between the manifolds with boundary $\overline{P_{C}\left(E_{-}\left(\Psi_{1}\right)\right)}$ and $\bar{B}$.

\subsubsection{Riemannian metric on $B$}

Since $B$ is an open subset of the vector space $W$, we may and shall identify the tangent space at a point of $B$ with $W$. We define a Riemannian metric $\tilde{\rho}$ on $B$ by

$$
\begin{aligned}
\tilde{\rho}(p)(X, Y) & :=\frac{\langle X, Y\rangle}{1-|p|^{2}}+\frac{\left\langle\beta_{3}(X, p), \beta_{3}(Y, p)\right\rangle}{\left(1-|p|^{2}\right)^{2}} \\
& =\frac{1}{\left(1-|p|^{2}\right)^{2}} \operatorname{Re}\left(\left(1-|p|^{2}\right) \beta_{3}(X, Y)+\beta_{1}\left(\beta_{3}(X, p), \beta_{3}(Y, p)\right)\right)
\end{aligned}
$$

for all $p \in B$ and all $X, Y \in T_{p} B=W$. Proposition 4.13 below shows that $\tilde{\rho}$ essentially coincides with the Riemannian metric defined in (2.11).

Lemma 4.12 Let $x, y \in W$. Then $\beta_{3}(x, y)=0$ if and only if $y \in(C x)^{\perp}$. 
Proof Let $\zeta, \eta, \xi \in C$. Proposition 4.4 yields

$$
\langle\zeta \bar{\eta}, \xi\rangle=\operatorname{Re} \beta_{1}(\zeta \bar{\eta}, \xi)=\operatorname{Re}(\zeta \bar{\eta} \bar{\xi})=\operatorname{Re}(\zeta \overline{\xi \eta})=\operatorname{Re} \beta_{1}(\zeta, \xi \eta)=\langle\zeta, \xi \eta\rangle .
$$

By Riesz' Representation Theorem we know that $\beta_{3}(x, y)=0$ if and only if

$$
\left\langle\beta_{3}(x, y), \xi\right\rangle=0 \text { for all } \xi \in C .
$$

Supposing that $x=(\zeta, v), y=(\eta, u) \in C \oplus V$, it follows from the definitions of $\beta_{3}, \beta_{1}, \beta_{2}$ and the inner product on $W$ that

$$
\begin{aligned}
\left\langle\beta_{3}(x, y), \xi\right\rangle & =\left\langle\beta_{1}(\zeta, \eta), \xi\right\rangle+\left\langle\beta_{2}(v, u), \xi\right\rangle=\langle\zeta \bar{\eta}, \xi\rangle+\langle\xi u, v\rangle \\
& =\langle\zeta, \xi \eta\rangle+\langle v, \xi u\rangle=\langle\bar{\xi} \zeta, \eta\rangle+\langle\bar{\xi} v, u\rangle \\
& =\langle\bar{\xi} x, y\rangle .
\end{aligned}
$$

Hence $\beta_{3}(x, y)=0$ if and only if $y \in(C x)^{\perp}$.

Proposition 4.13 The map $\widetilde{\rho}$ coincides, up to a multiplicative factor of 4 , with the Riemannian metric given by (2.11) on $B$.

Proof For $p=0$ and all $X, Y \in T_{0} B$ we have

$$
\widetilde{\rho}(0)(X, Y)=\langle X, Y\rangle=\frac{1}{4}\langle X, Y\rangle_{0-} .
$$

Suppose that $p=(\zeta, v) \in B \backslash\{0\}$. We claim that the equivalence class $C p$ (see Sect. 2.5) coincides with the $C$-orbit $C \cdot p$ of $p$. For $\zeta=0$ this is obviously true. Suppose that $\zeta \neq 0$. For each $\tau \in C \backslash\{0\}$, we have $\tau p=(\tau \zeta, \tau v)$ and

$$
(\tau \zeta)^{-1}(\tau v)=\zeta^{-1} \tau^{-1} \tau v=\zeta^{-1} v .
$$

Hence $(\zeta, v) \sim(\tau \zeta, \tau v)$. If $\tau=0$, then $\tau p=0 \in C p$ by definition. Conversely, suppose that $(\eta, u) \in C p \backslash\{0\}$. Then

$$
\eta \zeta^{-1} p=\eta \zeta^{-1}(\zeta, v)=\left(\eta, \eta \zeta^{-1} v\right)=\left(\eta, \eta \eta^{-1} u\right)=(\eta, u)
$$

Thus, $(\eta, u) \in C \cdot p$. Clearly, $0 \in C p \cap C \cdot p$. This shows that $C p=C \cdot p$.

Now let $p \in B \backslash\{0\}$ and $X, Y \in T_{p} B$. Suppose first that $X, Y \in(C p)^{\perp}$. Then Lemma 4.12 shows that

$$
\widetilde{\rho}(p)(X, Y)=\frac{\langle X, Y\rangle}{1-|p|^{2}}=\frac{1}{4}\langle X, Y\rangle_{p-} .
$$

Suppose now that $X \in C p$ and $Y \in(C p)^{\perp}$ (or vice versa). Then

$$
\widetilde{\rho}(p)(X, Y)=\frac{\langle X, Y\rangle}{1-|p|^{2}}=0=\frac{1}{4}\langle X, Y\rangle_{p-} .
$$

Finally suppose that $X, Y \in C p$. Then $X=\tau_{1} p$ and $Y=\tau_{2} p$ for some $\tau_{1}, \tau_{2} \in C$. From Lemma 2.12, Propositions 4.4 and 4.7 it follows that

$$
\begin{aligned}
\langle X, Y\rangle & =\operatorname{Re} \beta_{3}(X, Y)=\operatorname{Re} \beta_{3}\left(\tau_{1} p, \tau_{2} p\right)=\operatorname{Re}\left(\tau_{1} \beta_{3}(p, p) \bar{\tau}_{2}\right)=|p|^{2} \operatorname{Re}\left(\tau_{1} \bar{\tau}_{2}\right) \\
& =|p|^{2}\left\langle\tau_{1}, \tau_{2}\right\rangle .
\end{aligned}
$$


Then

$$
\begin{aligned}
\widetilde{\rho}(p)(X, Y) & =\frac{\langle X, Y\rangle}{1-|p|^{2}}+\frac{\left\langle\beta_{3}\left(\tau_{1} p, p\right), \beta_{3}\left(\tau_{2} p, p\right)\right\rangle}{\left(1-|p|^{2}\right)^{2}} \\
& =\frac{|p|^{2}\left\langle\tau_{1}, \tau_{2}\right\rangle}{1-|p|^{2}}+\frac{\left\langle\tau_{1} \beta_{3}(p, p), \tau_{2} \beta_{3}(p, p)\right\rangle}{\left(1-|p|^{2}\right)^{2}} \\
& =\frac{|p|^{2}\left\langle\tau_{1}, \tau_{2}\right\rangle}{1-|p|^{2}}+\frac{|p|^{4}\left\langle\tau_{1}, \tau_{2}\right\rangle}{\left(1-|p|^{2}\right)^{2}} \\
& =\frac{|p|^{2}\left\langle\tau_{1}, \tau_{2}\right\rangle}{\left(1-|p|^{2}\right)^{2}} \\
& =\frac{\langle X, Y\rangle}{\left(1-|p|^{2}\right)^{2}}=\frac{1}{4}\langle X, Y\rangle_{p-} .
\end{aligned}
$$

This completes the proof.

\subsubsection{Induced Riemannian isometries on B}

Let $\pi_{B}:=\tau_{B} \circ \pi: E_{-}\left(\Psi_{1}\right) \rightarrow B$ and suppose that $g \in U\left(\Psi_{1}, C\right)$. Since $g$ is $C$-linear, it induces a (unique) map $\widetilde{g}$ on $B$ by requiring the diagram

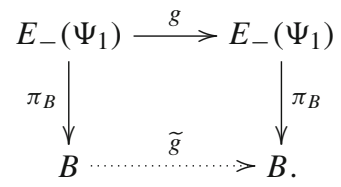

to commute. Our next goal is to show that each such induced map is a Riemannian isometry on $B$.

To simplify proofs we fix an orthonormal $C$-basis $\mathcal{B}(V):=\left\{v_{1}, \ldots, v_{n-1}\right\}$ for $V$. Then $\mathcal{B}(E):=\left\{e_{1}, e_{2}, \ldots, e_{n+1}\right\}$, given by

$$
e_{1}:=(1,0,0), \quad e_{2}:=(0,1,0), \quad e_{k}:=\left(0,0, v_{k-2}\right) \text { for } k=3, \ldots, n+1,
$$

is an orthonormal $C$-basis for $E$. In the following we will identify each element in $E, W$ and $V$, and each map $g \in U\left(\Psi_{1}, C\right)$ with its representative with respect to $\mathcal{B}(E)$. Since $E$ is a left $C$-module, the representing vector of $z \in E$ is a row, and the application of a map $g \in U\left(\Psi_{1}, C\right)$ to $z$ corresponds to the multiplication of the row vector of $z$ to the matrix of $g$ (and not vice versa, as usually in linear algebra). Further we use the notation $z^{*}$ for $\bar{z}^{\top}$ (the conjugate transpose of $z$ ).

Lemma 4.14 Let $v=\left(\zeta_{1}, \ldots, \zeta_{n-1}\right), u=\left(\eta_{1}, \ldots, \eta_{n-1}\right) \in V=C^{n-1}$. Then

$$
\beta_{2}(v, u)=\sum_{j=1}^{n-1} \zeta_{j} \overline{\eta_{j}}=v u^{*} .
$$

Proof Let $v_{j}, v_{k} \in \mathcal{B}(V)$. If $j=k$, then $\beta_{2}\left(v_{j}, v_{k}\right)=\left|v_{j}\right|^{2}=1$. If $j \neq k$, then $v_{j}$ is orthogonal to $C v_{k}$, hence

$$
\left\langle\beta_{2}\left(v_{j}, v_{k}\right), \xi\right\rangle=\left\langle\xi v_{k}, v_{j}\right\rangle=0
$$

for each $\xi \in C$, hence $\beta_{2}\left(v_{j}, v_{k}\right)=0$. The claim now follows by the $C$-sesquilinearity of $\beta_{2}$. 
Remark 4.15 Let $g \in U\left(\Psi_{1}, C\right)$ and suppose that

$$
g=\left(\begin{array}{cc}
a & b \\
c^{\top} & A
\end{array}\right)
$$

w.r.t. to $\mathcal{B}(E)$, where $a \in C, b, c \in C^{n}$ and $A \in C^{n \times n}$. Then the induced map $\tilde{g}: B \rightarrow B$ is given by

$$
\widetilde{g}(p)=\left(a+p c^{\top}\right)^{-1}(b+p A) .
$$

Further, the representing matrix of $\Psi_{1}$ is $\left({ }^{-1}{ }_{I}\right)$, where $I$ denotes the $n \times n$ identity matrix. Since $g$ preserves $\Psi_{1}$, we find the conditions

$$
\begin{aligned}
\left(\begin{array}{ll}
-1 & \\
& I
\end{array}\right) & =\left(\begin{array}{cc}
a & b \\
c^{\top} & A
\end{array}\right)\left(\begin{array}{cc}
-1 & \\
& I
\end{array}\right)\left(\begin{array}{cc}
\bar{a} & \bar{c} \\
b^{*} & A^{*}
\end{array}\right) \\
& =\left(\begin{array}{ll}
-|a|^{2}+|b|^{2} & -a \bar{c}+b A^{*} \\
-c^{\top} \bar{a}+A b^{*} & -c^{\top} \bar{c}+A A^{*}
\end{array}\right)
\end{aligned}
$$

on the entries of $g$.

The following proposition is proved by a long but straightforward calculation. We omit this proof here.

Proposition 4.16 Let $g \in U\left(\Psi_{1}, C\right)$. The induced map on $B$ is a Riemannian isometry.

In the following we will determine which elements in $U\left(\Psi_{1}, C\right)$ induce the same isometry on $B$. We denote the center of $C$ by $Z(C)$ and set

$$
Z^{1}(C):=\{a \in Z(C)|| a \mid=1\},
$$

which are the central elements in $C$ of unit length. Further we let $Z\left(\Psi_{1}, C\right)$ denote the center of $U\left(\Psi_{1}, C\right)$.

Lemma 4.17 We have $Z\left(\Psi_{1}, C\right)=\left\{a \operatorname{id}_{E} \mid a \in Z^{1}(C)\right\}$.

Proof Clearly, $\left\{a \operatorname{id}_{E} \mid a \in Z^{1}(C)\right\} \subseteq Z\left(\Psi_{1}, C\right)$. For the converse inclusion relation let

$$
g=\left(\begin{array}{cc}
a & b \\
c^{\top} & A
\end{array}\right) \in Z\left(\Psi_{1}, C\right)
$$

For each $d \in C,|d|=1$, and each matrix $D \in C^{n \times n}, D D^{*}=I$, the matrix

$$
h=\left(\begin{array}{ll}
d & 0 \\
0 & D
\end{array}\right)
$$

is in $U\left(\Psi_{1}, C\right)$. So necessarily,

$$
\left(\begin{array}{cc}
a d & b D \\
c^{\top} d & A D
\end{array}\right)=g h=h g=\left(\begin{array}{cc}
d a & d b \\
D c^{\top} & D A
\end{array}\right) .
$$

The left upper entries show that $a \in Z(C)$. Choosing different values for $d$, but the same (invertible) $D$, we find $b=c=0$. If $D$ runs through all permutation matrices and all rotation matrices, then we see that $A$ is a diagonal matrix $\operatorname{diag}(x, \ldots, x)$ with $x \in Z(C)$. By (4.4), $|a|=1=|x|$. Then

$$
g=\left(\begin{array}{cc}
a & 0 \\
0 & x I
\end{array}\right) .
$$


Let $h=\left(\begin{array}{cc}d & u \\ w^{\top} & B\end{array}\right) \in U\left(\Psi_{1}, C\right)$. Then

$$
\left(\begin{array}{cc}
a d & a u \\
x w^{\top} & x B
\end{array}\right)=g h=h g=\left(\begin{array}{cc}
d a & u x \\
w^{\top} a & B x
\end{array}\right) .
$$

Therefore, $a u=u x=x u$. For $u \neq 0$ it follows that $a=x$. Hence $g=a \operatorname{id}_{E}$ for some $a \in Z^{1}(C)$.

Proposition 4.18 Let $g_{1}, g_{2} \in U\left(\Psi_{1}, C\right)$. Then $g_{1}$ and $g_{2}$ induce the same isometry on $B$ if and only if $g_{1} h=g_{2}$ for some $h \in Z\left(\Psi_{1}, C\right)$.

Proof It suffices to show that exactly the elements in $Z\left(\Psi_{1}, C\right)$ induce id ${ }_{B}$. Let $g$ be an element of $U\left(\Psi_{1}, C\right)$ and suppose that

$$
g=\left(\begin{array}{cc}
a & b \\
c^{\top} & A
\end{array}\right)
$$

w.r.t. $\mathcal{B}(E)$, where $a \in C, b, c \in C^{n}$ and $A \in C^{n \times n}$. Then the induced isometry on $B$ is

$$
\tilde{g}:\left\{\begin{array}{l}
B \rightarrow B \\
p \mapsto\left(a+p c^{\top}\right)^{-1}(b+p A) .
\end{array}\right.
$$

Suppose that $\widetilde{g}=\operatorname{id}_{B}$. Then

$$
0=\widetilde{g}(0)=a^{-1} b,
$$

which yields that $b=0$ and, by (4.4), $|a|=1$. Hence

$$
g=\left(\begin{array}{cc}
a & 0 \\
c^{\top} & A
\end{array}\right) .
$$

Now (4.4) shows that $0=-a \bar{c}$, which implies that $c=0$. Thus,

$$
\widetilde{g}(p)=a^{-1} p A
$$

for all $p \in B$. Suppose that $A=\left(a_{i j}\right)_{i, j=1, \ldots, n}$. Let $j \in\{1, \ldots, n\}$ and consider the vector $p=\left(p_{i}\right)_{i=1, \ldots, n}$ with $p_{j}=\frac{1}{2}$ and $p_{i}=0$ for $i \neq j$. Then $p \in B$ and

$$
p=\widetilde{g}(p)=\frac{1}{2} a^{-1}\left(a_{j 1}, \ldots, a_{j n}\right) .
$$

Therefore, $a_{i j}=0$ for $i \neq j$ and $a_{j j}=a$. This shows that $A=a I$.

Now let $\zeta \in C$ with $|\zeta|<1$. Then $(\zeta, 0) \in B$ and therefore,

$$
(\zeta, 0)=\tilde{g}(\zeta, 0)=a^{-1}(\zeta, 0) a .
$$

Hence $a \zeta=\zeta a$. By scaling, this equality holds for all $\zeta \in C$. Thus $a \in Z^{1}(C)$, which yields that $g \in Z\left(\Psi_{1}, C\right)$. Conversely, each element of $Z\left(\Psi_{1}, C\right)$ clearly induces $\operatorname{id}_{B}$ on $B$.

Let

$$
\mathrm{PU}\left(\Psi_{1}, C\right):=U\left(\Psi_{1}, C\right) / Z\left(\Psi_{1}, C\right)
$$

and denote the coset of $g \in U\left(\Psi_{1}, C\right)$ by $[g]$. As before we use the notation $\widetilde{g}$ for the isometry on $B$ induced by $g \in U\left(\Psi_{1}, C\right)$. Recall that $G$ is the full isometry group of $B$. 
Corollary 4.19 The map

$$
j_{\Psi_{1}}: \begin{cases}\mathrm{PU}\left(\Psi_{1}, C\right) & \rightarrow G \\ {[g]} & \mapsto \widetilde{g}\end{cases}
$$

is a monomorphism of groups.

The next goal is to characterize the subgroup of $G$ to which $\mathrm{PU}\left(\Psi_{1}, C\right)$ is isomorphic. For this it is most convenient to work with the space $\overline{P_{C}\left(E_{-}\left(\Psi_{2}\right)\right)}$.

4.4.4 Set of representatives for $\overline{P_{C}\left(E_{-}\left(\Psi_{2}\right)\right)}$

If $z=(\zeta, \eta, v) \in E_{-}\left(\Psi_{2}\right)$, then

$$
0>q_{2}((\zeta, \eta, v))=-2\langle\zeta, \eta\rangle+|v|^{2},
$$

which shows that $\zeta \neq 0$. Thus, $[z] \in P_{C}\left(E_{-}\left(\Psi_{2}\right)\right)$ is represented by $\left(1, \zeta^{-1} \eta, \zeta^{-1} v\right)$ and this is the unique representative of the form $(1, *, *)$. We note that

$$
0>q_{2}\left(\left(1, \zeta^{-1} \eta, \zeta^{-1} v\right)\right)=-2\left\langle 1, \zeta^{-1} \eta\right\rangle+\left|\zeta^{-1} v\right|^{2}=-2 \operatorname{Re}\left(\zeta^{-1} \eta\right)+\left|\zeta^{-1} v\right|^{2},
$$

and therefore

$$
\operatorname{Re}\left(\zeta^{-1} \eta\right)>\frac{1}{2}\left|\zeta^{-1} v\right|^{2}
$$

Hence, if we define

$$
H:=\left\{\left.(\tau, u) \in C \oplus V\left|\operatorname{Re}(\tau)>\frac{1}{2}\right| u\right|^{2}\right\},
$$

then

$$
\left(\zeta^{-1} \eta, \zeta^{-1} v\right) \in H
$$

Conversely, if $(\tau, u) \in H$, then $[(1, \tau, u)] \in P_{C}\left(E_{-}\left(\Psi_{2}\right)\right)$.

If $z=(\zeta, \eta, v) \in E_{0}\left(\Psi_{2}\right)$, then either $\zeta \neq 0$ or $z=(0, \eta, 0)$ with $\eta \neq 0$. Applying the previous argumentation we see that the set of elements in $P_{C}\left(E_{0}\left(\Psi_{2}\right)\right)$ which have a representative $(\zeta, \eta, v) \in E_{0}\left(\Psi_{2}\right)$ with $\zeta \neq 0$ is bijective to

$$
\left\{\left.(\tau, u) \in C \oplus V\left|\operatorname{Re}(\tau)=\frac{1}{2}\right| u\right|^{2}\right\}
$$

via

$$
[(\zeta, \eta, v)] \mapsto\left(\zeta^{-1} \eta, \zeta^{-1} v\right)
$$

If $z=(0, \eta, 0)$ with $\eta \neq 0$, then $[z]$ is represented by $(0,1,0)$. Let

$$
\bar{H}^{g}:=\left\{\left.(\tau, u) \in C \oplus V\left|\operatorname{Re}(\tau) \geq \frac{1}{2}\right| u\right|^{2}\right\} \cup\{\infty\}
$$

denote the closure of $H$ in the one-point compactification $(C \oplus V) \cup\{\infty\}$ of $C \oplus V$. Then the map $\tau_{H}: \overline{P_{C}\left(E_{-}\left(\Psi_{2}\right)\right)} \rightarrow \bar{H}^{g}$,

$$
\tau_{H}([(\zeta, \eta, v)]):= \begin{cases}\left(\zeta^{-1} \eta, \zeta^{-1} v\right) & \text { if } \zeta \neq 0 \\ \infty & \text { if } \zeta=0\end{cases}
$$


is a bijection with inverse map

$$
\tau_{H}^{-1}(\infty)=[(0,1,0)] \quad \tau_{H}^{-1}(\tau, u)=[(1, \tau, u)] .
$$

Since $\left.\tau_{H}\right|_{P_{C}\left(E_{-}\left(\Psi_{2}\right)\right)}$ is a restriction of the chart map $\varphi_{1}$ from Sect. 4.3, $\tau_{H}$ is a diffeomorphism between $P_{C}\left(E_{-}\left(\Psi_{2}\right)\right)$ and $H$.

\subsubsection{Riemannian metric and induced isometries on $H$}

Recall the model $D$ from Sect. 2.4. The map

$$
\beta: \begin{cases}\bar{H}^{g} & \rightarrow \bar{D}^{g} \\ \infty & \mapsto \infty \\ (\zeta, v) & \mapsto(\zeta, \sqrt{2} v)\end{cases}
$$

is clearly a diffeomorphism between the manifolds $\bar{H}^{g}$ and $\bar{D}^{g}$ with boundary, and hence a diffeomorphism between $H$ and $D$. We endow $H$ with a Riemannian metric by requiring that $\beta$ be an isometry.

Let $\pi_{H}:=\tau_{H} \circ \pi: E_{-}\left(\Psi_{2}\right) \rightarrow H$. As before, each $g \in U\left(\Psi_{2}, C\right)$ defines a (unique) map $\widetilde{g}$ on $H$ which makes the diagram

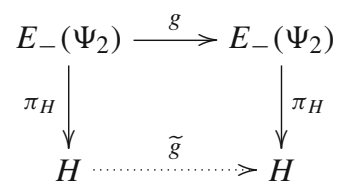

commutative.

Let $Z\left(\Psi_{2}, C\right)$ denote the center of $U\left(\Psi_{2}, C\right)$ and set

$$
\mathrm{PU}\left(\Psi_{2}, C\right):=U\left(\Psi_{2}, C\right) / Z\left(\Psi_{2}, C\right) .
$$

In the following we will use the results from the previous subsections to show that $\operatorname{PU}\left(\Psi_{2}, C\right)$ is isomorphic to a subgroup of the full isometry group $G$ of $H$. We consider the map

$$
T: \begin{cases}E & \rightarrow E \\ (\zeta, \eta, v) & \mapsto\left(\frac{1}{\sqrt{2}}(\zeta-\eta), \frac{1}{\sqrt{2}}(\zeta+\eta), v\right) .\end{cases}
$$

Then $T$ is $C$-linear and invertible with inverse map $T^{-1}: E \rightarrow E$,

$$
T^{-1}(\zeta, \eta, v)=\left(\frac{1}{\sqrt{2}}(\zeta+\eta), \frac{1}{\sqrt{2}}(-\zeta+\eta), v\right) .
$$

The following lemma is shown by a straightforward calculation.

Lemma 4.20 The map $T$ transforms $\Psi_{2}$ into $\Psi_{1}$, i.e., $\Psi_{2} \circ(T \times T)=\Psi_{1}$. Further, we have $T\left(E_{-}\left(\Psi_{1}\right)\right)=E_{-}\left(\Psi_{2}\right)$ and $T\left(E_{0}\left(\Psi_{1}\right)\right)=E_{0}\left(\Psi_{2}\right)$.

Proposition 4.21 The map

$$
\lambda: \begin{cases}U\left(\Psi_{1}, C\right) & \rightarrow U\left(\Psi_{2}, C\right) \\ g & \mapsto T \circ g \circ T^{-1}\end{cases}
$$

is an isomorphism with $\lambda\left(Z\left(\Psi_{1}, C\right)\right)=Z\left(\Psi_{2}, C\right)$. 
Proof Let $g \in U\left(\Psi_{1}, C\right)$. Since $T \in \mathrm{GL}_{C}(E)$, we have $\lambda(g) \in \mathrm{GL}_{C}(E)$. Lemma 4.20 shows that

$$
\begin{aligned}
\Psi_{2} \circ\left(T g T^{-1} \times T g T^{-1}\right) & =\Psi_{2} \circ(T \times T) \circ\left(g T^{-1} \times g T^{-1}\right) \\
& =\Psi_{1} \circ(g \times g) \circ\left(T^{-1} \times T^{-1}\right)=\Psi_{1} \circ\left(T^{-1} \times T^{-1}\right) \\
& =\Psi_{2} .
\end{aligned}
$$

Hence $\lambda(g) \in U\left(\Psi_{2}, C\right)$. Clearly, $\lambda$ is an isomorphism of groups, which shows that $\lambda\left(Z\left(\Psi_{1}, C\right)\right)=Z\left(\Psi_{2}, C\right)$.

For the proof of the following proposition recall the Cayley transform $\mathcal{C}: B \rightarrow D$ from Sect. 2.8. For each $\zeta \in C \backslash\{1\}$ we have

$$
\begin{aligned}
(1-\zeta)^{-1}(1+\zeta) & =|1-\zeta|^{-2}(1-\bar{\zeta})(1+\zeta)=|1-\zeta|^{-2}\left(1-\bar{\zeta}+\zeta-|\zeta|^{2}\right) \\
& =|1-\zeta|^{-2}\left(1+2 \operatorname{Im} \zeta-|\zeta|^{2}\right) .
\end{aligned}
$$

Hence

$$
\mathcal{C}(\zeta, v)=(1-\zeta)^{-1}(1+\zeta, 2 v) .
$$

Proposition 4.22 Let $g \in U\left(\Psi_{2}, C\right)$. Then the induced map $\widetilde{g}$ on $H$ is an isometry. Further, the map

$$
j_{\Psi_{2}}: \begin{cases}\mathrm{PU}\left(\Psi_{2}, C\right) & \rightarrow G \\ {[g]} & \mapsto \widetilde{g}\end{cases}
$$

is well-defined and a monomorphism of groups.

Proof The map $T$ induces the map $\widehat{T}: P_{C}\left(E_{-}\left(\Psi_{1}\right)\right) \rightarrow P_{C}\left(E_{-}\left(\Psi_{2}\right)\right)$ given by

$$
\widehat{T}([(\zeta, \eta, v)])=\left[\left(\frac{1}{\sqrt{2}}(\zeta-\eta), \frac{1}{\sqrt{2}}(\zeta+\eta), v\right)\right]
$$

and the map $\widetilde{T}: B \rightarrow H$ given by

$$
\begin{aligned}
\widetilde{T}((\eta, v)) & =\tau_{H}(\widehat{T}[(1, \eta, v)])=\tau_{H}\left(\left[\frac{1}{\sqrt{2}}(1-\eta), \frac{1}{\sqrt{2}}(1+\eta), v\right]\right) \\
& =\tau_{H}\left(\left[\left(1,(1-\eta)^{-1}(1+\eta),(1-\eta)^{-1} \sqrt{2} v\right)\right]\right) \\
& =(1-\eta)^{-1}(1+\eta, \sqrt{2} v) .
\end{aligned}
$$

Therefore, $\widetilde{T}=\beta^{-1} \circ \mathcal{C}$. From Propositions 2.9 and 4.13 it follows that the isometry group of $B$ and that of $H$ are identical. Then Propositions 4.16 and 4.21 imply that for each $g \in U\left(\Psi_{2}, C\right)$, the induced map $\widetilde{g}$ is an isometry on $H$. Moreover, Proposition 4.21 shows that $\lambda$ factors to a map

$$
\tilde{\lambda}: \operatorname{PU}\left(\Psi_{1}, C\right) \rightarrow \operatorname{PU}\left(\Psi_{2}, C\right) .
$$

Recall the map $j_{\Psi_{1}}$ from Corollary 4.19. Then the diagram

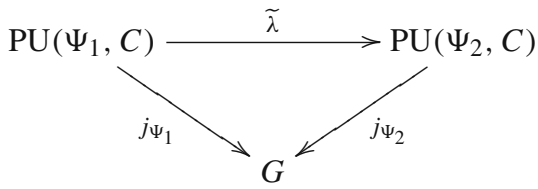

commutes, which completes the proof. 


\subsubsection{Lifted isometries}

In this section, we determine which isometries on $H$ are induced from an element in $U\left(\Psi_{2}, C\right)$. To that end we need explicit formulas for the action of an element $g \in G$ on $H$, which are provided by the following remark.

Remark 4.23 Let $g \in G$. Section 2.8 provides explicit formulas for the action of $g$ on $D$. Using the isometry $\beta: H \rightarrow D$, the action of $g$ on $H$ is given by

$$
g^{H}:=\beta^{-1} \circ g \circ \beta: H \rightarrow H .
$$

Evaluating this formula, we find the following action laws. For the geodesic inversion $\sigma$ we have

$$
\sigma^{H}(\zeta, v)=\zeta^{-1}(1,-v)
$$

For $a_{s} \in A$ we get

$$
a_{s}^{H}(\zeta, v)=\left(s \zeta, s^{1 / 2} v\right)
$$

For $n=(\xi, w) \in N$ it follows

$$
n^{H}(\zeta, v)=\left(\zeta+\xi+\frac{1}{4}|w|^{2}+\beta_{2}\left(v, \frac{1}{\sqrt{2}} w\right), \frac{1}{\sqrt{2}} w+v\right) .
$$

For $m=(\varphi, \psi) \in M$ we have

$$
m^{H}(\zeta, v)=(\varphi(\zeta), \psi(v))
$$

Proposition 4.24 A representative of $\sigma^{H}$ in $U\left(\Psi_{2}, C\right)$ is

$$
g(\zeta, \eta, v)=(\eta, \zeta,-v)
$$

Proposition 4.25 Let $a_{s} \in A$. Then

$$
g(\zeta, \eta, v)=\left(s^{-1 / 2} \zeta, s^{1 / 2} \eta, v\right)
$$

is a representative of $a_{s}$ in $U\left(\Psi_{2}, C\right)$.

Proof Obviously, $g$ is $C$-linear and induces $a_{s}$ on $B$. Further

$$
\begin{aligned}
\Psi_{2}\left(g\left(\zeta_{1}, \eta_{1}, v_{1}\right), g\left(\zeta_{2}, \eta_{2}, v_{2}\right)\right) & =\Psi_{2}\left(\left(s^{-1 / 2} \zeta_{1}, s^{1 / 2} \eta_{1}, v_{1}\right),\left(s^{-1 / 2} \zeta_{2}, s^{1 / 2} \eta_{2}, v_{2}\right)\right) \\
& =-s^{-1 / 2} \zeta_{1} \overline{\eta_{2}} s^{1 / 2}-s^{-1 / 2} \eta_{1} \overline{\zeta_{2}} s^{1 / 2}+\beta_{2}\left(v_{1}, v_{2}\right) \\
& =-\zeta_{1} \overline{\eta_{2}}-\eta_{1} \bar{\zeta}_{2}+\beta_{2}\left(v_{1}, v_{2}\right) \\
& =\Psi_{2}\left(\left(\zeta_{1}, \eta_{1}, v_{1}\right),\left(\zeta_{2}, \eta_{2}, v_{2}\right)\right)
\end{aligned}
$$

Hence $g \in U\left(\Psi_{2}, C\right)$.

Proposition 4.26 Let $n=(\xi, w) \in N$. A representative of $n$ in $U\left(\Psi_{2}, C\right)$ is

$$
g(\zeta, \eta, v)=\left(\zeta, \zeta\left(\xi+\frac{1}{4}|w|^{2}\right)+\eta+\frac{1}{\sqrt{2}} \beta_{2}(v, w), \frac{1}{\sqrt{2}} \zeta w+v\right)
$$


Proof The map $g$ is clearly $C$-linear and induces $n$ on $B$. To see that $\Psi_{2}$ is $g$-invariant, we calculate

$$
\begin{aligned}
\Psi_{2} & \left(g\left(\zeta_{1}, \eta_{1}, v_{1}\right), g\left(\zeta_{2}, \eta_{2}, v_{2}\right)\right) \\
= & \Psi_{2}\left[\left(\zeta_{1}, \zeta_{1}\left(\xi+\frac{1}{4}|w|^{2}\right)+\eta_{1}+\frac{1}{\sqrt{2}} \beta_{2}\left(v_{1}, w\right), \frac{1}{\sqrt{2}} \zeta_{1} w+v_{1}\right),\right. \\
& \left.\left(\zeta_{2}, \zeta_{2}\left(\xi+\frac{1}{4}|w|^{2}\right)+\eta_{2}+\frac{1}{\sqrt{2}} \beta_{2}\left(v_{2}, w\right), \frac{1}{\sqrt{2}} \zeta_{2} w+v_{2}\right)\right] \\
= & -\zeta_{1}\left[\left(-\xi+\frac{1}{4}|w|^{2}\right) \bar{\zeta}_{2}+\bar{\eta}_{2}+\frac{1}{\sqrt{2}} \beta_{2}\left(w, v_{2}\right)\right] \\
& -\left[\zeta_{1}\left(\xi+\frac{1}{4}|w|^{2}\right)+\eta_{1}+\frac{1}{\sqrt{2}} \beta_{2}\left(v_{1}, w\right)\right] \bar{\zeta}_{2}+\beta_{2}\left(\frac{1}{\sqrt{2}} \zeta_{1} w+v_{1}, \frac{1}{\sqrt{2}} \zeta_{2} w+v_{2}\right) \\
= & \zeta_{1} \xi \bar{\zeta}_{2}-\frac{1}{4}|w|^{2} \zeta_{1} \bar{\zeta}_{2}-\zeta_{1} \bar{\eta}_{2}-\frac{1}{\sqrt{2}} \zeta_{1} \beta_{2}\left(w, v_{2}\right)-\zeta_{1} \xi \bar{\zeta}_{2}-\frac{1}{4}|w|^{2} \zeta_{1} \bar{\zeta}_{2} \\
& -\eta_{1} \bar{\zeta}_{2}-\frac{1}{\sqrt{2}} \beta_{2}\left(v_{1}, w\right) \bar{\zeta}_{2}+\frac{1}{2}|w|^{2} \zeta_{1} \bar{\zeta}_{2}+\frac{1}{\sqrt{2}} \zeta_{1} \beta_{2}\left(w, v_{2}\right) \\
& +\frac{1}{\sqrt{2}} \beta_{2}\left(v_{1}, w\right) \bar{\zeta}_{2}+\beta_{2}\left(v_{1}, v_{2}\right) \\
= & \Psi_{2}\left(\left(\zeta_{1}, \eta_{1}, v_{1}\right),\left(\zeta_{2}, \eta_{2}, v_{2}\right)\right) .
\end{aligned}
$$

This completes the proof.

The remaining part of this section is devoted to the discussion which elements of $M$ have a representative in $U\left(\Psi_{2}, C\right)$. The situation for $M$ is much more involved than the proofs of Propositions 4.24-4.26. In particular, it will turn out that in general not every element of $M$ can be lifted to $U\left(\Psi_{2}, C\right)$.

Lemma 4.27 Let $m=(\varphi, \psi) \in M$ and suppose that $\varphi$ is an inner automorphism of $C$. Then

$$
\left\{\beta_{2}\left(\psi\left(v_{1}\right), \psi\left(v_{2}\right)\right) \mid v_{1}, v_{2} \in V\right\}=C .
$$

Proof Let $a \in C \backslash\{0\}$ and suppose that $\varphi(\zeta)=a^{-1} \zeta a$ for all $\zeta \in C$. Choose $v \in V$ with $|v|=1$. For each $\zeta \in C$ we find

$$
\begin{aligned}
\beta_{2}\left(\psi\left(a \zeta a^{-1} v\right), \psi(v)\right) & =\beta_{2}\left(\varphi\left(a \zeta a^{-1}\right) \psi(v), \psi(v)\right)=\beta_{2}(\zeta \psi(v), \psi(v)) \\
& =\zeta \beta_{2}(\psi(v), \psi(v))=\zeta|\psi(v)|^{2}=\zeta|v|^{2} \\
& =\zeta .
\end{aligned}
$$

Therefore,

$$
C \subseteq\left\{\beta_{2}\left(\psi\left(v_{1}\right), \psi\left(v_{2}\right)\right) \mid v_{1}, v_{2} \in V\right\} .
$$

The converse inclusion relation clearly holds by the range of $\beta_{2}$.

Let $i_{H}: \bar{H}^{g} \rightarrow E_{-}\left(\Psi_{2}\right) \cup E_{0}\left(\Psi_{2}\right)$ be any section of

$$
\pi_{H}=\tau_{H} \circ \pi: E_{-}\left(\Psi_{2}\right) \cup E_{0}\left(\Psi_{2}\right) \rightarrow \bar{H}^{g} .
$$


Let $\widetilde{g} \in G$ and recall from Proposition 2.14 that $g$ extends continuously to $\bar{H}^{g}$. If the element $g \in U\left(\Psi_{2}, C\right)$ is a representative of $\widetilde{g}$, then the diagram

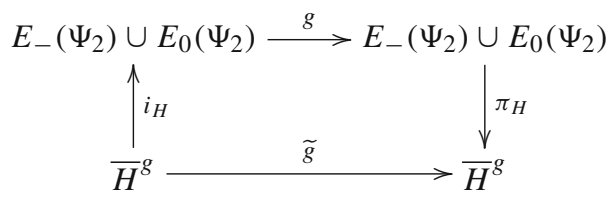

commutes. We will make use of this fact in the proof of Proposition 4.28 below. For convenience we define $i_{H}$ by

$$
i_{H}(\infty):=(0,1,0) \text { and } i_{H}(\eta, v):=(1, \eta, v) .
$$

Proposition 4.28 Let $m=(\varphi, \psi) \in M$. Then there exists a representative of $m$ in $U\left(\Psi_{2}, C\right)$ if and only if $\varphi=\mathrm{id}$. In this case,

$$
g(\zeta, \eta, v)=(\zeta, \eta, \psi(v))
$$

is such a representative.

Proof Suppose first that $m=(\varphi, \psi)$ with $\varphi=$ id. We will show that

$$
g: \begin{cases}E & \rightarrow E \\ (\zeta, \eta, v) & \mapsto(\zeta, \eta, \psi(v))\end{cases}
$$

is an element of $U\left(\Psi_{2}, C\right)$. To that end let $\left(\zeta_{1}, \eta_{1}, v_{1}\right),\left(\zeta_{2}, \eta_{2}, v_{2}\right) \in E$ and $\zeta \in C$. Then

$$
\begin{aligned}
g\left(\zeta\left(\zeta_{1}, \eta_{1}, v_{1}\right)+\left(\zeta_{2}, \eta_{2}, v_{2}\right)\right) & =\left(\zeta \zeta_{1}+\zeta_{2}, \zeta \eta_{1}+\eta_{2}, \psi\left(\zeta v_{1}+v_{2}\right)\right) \\
& =\left(\zeta \zeta_{1}, \zeta \eta_{1}, \psi\left(\zeta v_{1}\right)\right)+\left(\zeta_{2}, \eta_{2}, \psi\left(v_{2}\right)\right) \\
& =\left(\zeta \zeta_{1}, \zeta \eta_{1}, \varphi(\zeta) \psi\left(v_{1}\right)\right)+g\left(\zeta_{2}, \eta_{2}, v_{2}\right) \\
& =\left(\zeta \zeta_{1}, \zeta \eta_{1}, \zeta \psi\left(v_{1}\right)\right)+g\left(\zeta_{2}, \eta_{2}, v_{2}\right) \\
& =\zeta g\left(\zeta_{1}, \eta_{1}, v_{1}\right)+g\left(\zeta_{2}, \eta_{2}, v_{2}\right) .
\end{aligned}
$$

This shows that $g$ is $C$-linear. The map $g$ is obviously invertible, hence $g \in \mathrm{GL}_{C}(E)$. Lemma 3.14(ii) implies that $\Psi_{2}$ is invariant under $g$. Hence $g \in U\left(\Psi_{2}, C\right)$. Clearly, $g$ induces $m$.

Suppose now that $m=(\varphi, \psi) \in M$ and that there is a representative $g$ of $m$ in $U\left(\Psi_{2}, C\right)$. We have to show that $\varphi=$ id. Since $m(0)=0$, it follows that

$$
g\left(i_{H}(0)\right)=g(1,0,0) \in \pi_{H}^{-1}(0)=(C \backslash\{0\}) \times\{0\} \times\{0\} .
$$

Thus, there is $a \in C \backslash\{0\}$ such that $g(1,0,0)=(a, 0,0)$. Further $m(\infty)=\infty$. The same argument shows that there is $b \in C \backslash\{0\}$ such that

$$
g\left(i_{H}(\infty)\right)=g(0,1,0)=(0, b, 0) .
$$

Then for each $\zeta \in C$ with $\operatorname{Re} \zeta \geq 0$ we have

$$
\begin{aligned}
(\varphi(\zeta), 0) & =m(\zeta, 0)=\pi_{H}\left(g\left(i_{H}(\zeta, 0)\right)\right)=\pi_{H}(g(1, \zeta, 0))=\pi_{H}((a, \zeta b, 0)) \\
& =\left(a^{-1} \zeta b, 0\right) .
\end{aligned}
$$

Thus $\varphi(\zeta)=a^{-1} \zeta b$ for all $\zeta \in C$ with $\operatorname{Re} \zeta \geq 0$. Now

$$
1=\varphi(1)=a^{-1} b
$$


and therefore $b=a$. Hence $\varphi(\zeta)=a^{-1} \zeta a$ for all $\zeta \in C$ with $\operatorname{Re} \zeta \geq 0$. Suppose now that $(\eta, v) \in H$ is arbitrary. Then

$$
\begin{aligned}
g\left(i_{H}(\eta, v)\right) & =g(1, \eta, v)=g(1, \eta, 0)+g(0,0, v) \\
& =(a, \eta a, 0)+(\sigma, \tau, u)=(a+\sigma+\eta a+\tau, u)
\end{aligned}
$$

where $(\sigma, \tau, u)$ depends only on $v$. From

$$
\begin{aligned}
\left(a^{-1} \eta a, \psi(v)\right) & =(\varphi(\eta), \psi(v))=\pi_{H}(a+\sigma, \eta a+\tau, u) \\
& =\left((a+\sigma)^{-1}(\eta a+\tau),(a+\sigma)^{-1} u\right)
\end{aligned}
$$

it follows that

$$
a^{-1} \eta a=(a+\sigma)^{-1} \eta a+(a+\sigma)^{-1} \tau
$$

for all $\eta$ with $\operatorname{Re}(\eta)>\frac{1}{2}|v|^{2}$. Pick $k \in \mathbb{R}$ with $k>\frac{1}{2}|v|^{2}$. Then

$$
k=a^{-1} k a=k(a+\sigma)^{-1} a+(a+\sigma)^{-1} \tau
$$

and

$$
2 k=2 k(a+\sigma)^{-1} a+(a+\sigma)^{-1} \tau .
$$

Solving this system for $\sigma$ and $\tau$, we find $\sigma=\tau=0$. Hence

$$
g(1, \eta, v)=(a, \eta a, a \psi(v)) .
$$

Since $g$ has to be $C$-linear, it follows that

$$
g(\zeta, \eta, v)=(\zeta a, \eta a, a \psi(v))
$$

for all $(\zeta, \eta, v) \in E$. We derive further properties of $a$. Let $v \in V \backslash\{0\}$. Then $g$ being in $U\left(\Psi_{2}, C\right)$ yields that

$$
|v|^{2}=q_{2}((0,0, v))=q_{2}(g(0,0, v))=|a \psi(v)|^{2}=|a|^{2}|\psi(v)|^{2}=|a|^{2}|\psi(v)|^{2},
$$

hence $|a|^{2}=1$. Again using that $g \in U\left(\Psi_{2}, C\right)$ we find that for all $v_{1}, v_{2} \in V$

$$
\begin{aligned}
\beta_{2}\left(\psi\left(v_{1}\right), \psi\left(v_{2}\right)\right) & =\Psi_{2}\left(\left(0,0, v_{1}\right),\left(0,0, v_{2}\right)\right) \\
& =\Psi_{2}\left(g\left(0,0, v_{1}\right), g\left(0,0, v_{2}\right)\right)=\Psi_{2}\left(\left(0,0, a \psi\left(v_{1}\right)\right),\left(0,0, a \psi\left(v_{2}\right)\right)\right) \\
& =\beta_{2}\left(a \psi\left(v_{1}\right), a \psi\left(v_{2}\right)\right)=a \beta_{2}\left(\psi\left(v_{1}\right), \psi\left(v_{2}\right)\right) \bar{a} \\
& =a \beta_{2}\left(\psi\left(v_{1}\right), \psi\left(v_{2}\right)\right) a^{-1} .
\end{aligned}
$$

Before we can apply Lemma 4.27 we have to show that $\varphi(\zeta)=a^{-1} \zeta a$ for all $\zeta \in C$. Let $\zeta \in C$ with $\operatorname{Re} \zeta<0$ and consider the decomposition $\zeta=\zeta_{1}+\zeta_{2}$ with $\zeta_{1} \in \mathbb{R}$ and $\zeta_{2} \in C^{\prime}$. Then the $\mathbb{R}$-linearity of $\varphi$ yields

$$
\begin{aligned}
\varphi(\zeta) & =\varphi\left(\zeta_{1}+\zeta_{2}\right)=\varphi\left(\zeta_{1}\right)+\varphi\left(\zeta_{2}\right)=-\varphi\left(-\zeta_{1}\right)+a^{-1} \zeta_{2} a \\
& =-a^{-1}\left(-\zeta_{1}\right) a+a^{-1} \zeta_{2} a=a^{-1} \zeta_{1} a+a^{-1} \zeta_{2} a=a^{-1}\left(\zeta_{1}+\zeta_{2}\right) a=a^{-1} \zeta a .
\end{aligned}
$$

Then Lemma 4.27 implies that $a \in Z(C)$. Therefore, $\varphi=$ id. 
We set

$$
M^{\mathrm{res}}:=\{(\varphi, \psi) \in M \mid \varphi=\mathrm{id}\} \text { and } G^{\mathrm{res}}:=N \sigma M^{\mathrm{res}} A N \cup M^{\mathrm{res}} A N .
$$

Further we define a map $\varphi_{H}: G^{\text {res }} \rightarrow \mathrm{PU}\left(\Psi_{2}, C\right)$ as follows: For $\widetilde{g}=\sigma, \widetilde{g} \in A, \widetilde{g} \in N$ or $\widetilde{g} \in M^{\text {res }}$ we set $\varphi_{H}(\widetilde{g}):=[g]$, where $g$ is the lift of $\widetilde{g}$ as in Propositions 4.24-4.26 or 4.28, respectively. For $\widetilde{g}=n_{2} \sigma m a_{s} n_{1} \in N \sigma M^{\text {res }} A N$ we define

$$
\varphi_{H}(\widetilde{g}):=\varphi_{H}\left(n_{2}\right) \varphi_{H}(\sigma) \varphi_{H}(m) \varphi_{H}\left(a_{s}\right) \varphi_{H}\left(n_{1}\right),
$$

and likewise for $\widetilde{g}=m a_{s} n \in M^{\text {res }} A N$ we set

$$
\varphi_{H}(\widetilde{g}):=\varphi_{H}(m) \varphi_{H}\left(a_{s}\right) \varphi_{H}(n) .
$$

In other words, we extend $\varphi_{H}$ to a group homomorphism. Since the Bruhat decomposition of an element $\widetilde{g} \in G^{\text {res }}$ is unique and the Bruhat decomposition of $G^{\text {res }}$ can be directly transfered to $\mathrm{PU}\left(\Psi_{2}, C\right)$, the map $\varphi_{H}$ is indeed well-defined. By our previous considerations, $\varphi_{H}$ is even a group isomorphism.

The following remark shows that $M^{\text {res }}$ is not necessarily all of $M$.

Remark 4.29 The classification [15, Theorem 3.1] of division algebras arising from associative $J^{2} C$-module structures $(C, V, J)$ shows that $C$ is either real or complex or quaternionic numbers. In the following we show that $M=M^{\text {res }}$ for $C=\mathbb{R}$, but $M \neq M^{\text {res }}$ for $C=\mathbb{C}$ or $C=\mathbb{H}$ (quaternions).

(i) Let $C=\mathbb{R}$ and suppose that $m=(\varphi, \psi) \in M$. We claim that $\varphi=$ id. Since $\varphi: \mathbb{R} \rightarrow \mathbb{R}$ is a norm-preserving endomorphism of $\mathbb{R}$, the map $\varphi$ is either id or $-\mathrm{id}$. Assume for contradiction that $\varphi=-$ id. Let $(\zeta, v) \in C \times V$ such that $\zeta \neq 0$ and $v \neq 0$. Then

$$
\varphi(\zeta) \psi(v)=-\zeta \psi(v)=-\psi(\zeta v) \neq \psi(\zeta v) .
$$

Hence $m \notin M$. This is a contradiction and therefore $\varphi=\mathrm{id}$.

(ii) Let $C=\mathbb{C}=V$ and suppose that $\varphi=\psi$ are complex conjugation. For all elements $(\zeta, v) \in C \oplus V=\mathbb{C}^{2}$ we have $\varphi(\zeta) \psi(v)=\bar{\zeta} \bar{v}=\overline{\zeta v}=\psi(\zeta v)$. Clearly, $\varphi, \psi$ are $\mathbb{R}$-linear endomorphisms of the Euclidean vector space $\mathbb{C}$. Therefore, $m=(\varphi, \psi) \in M$, but $m \notin M^{\text {res }}$.

(iii) Let $C=\mathbb{H}=V$. Define $\varphi: C \rightarrow C$ and $\psi: V \rightarrow V$ by

$$
\begin{gathered}
\varphi(a+i b+j c+k d):=a-i b-j c+k d \\
\psi(a+i b+j c+k d):=-a+i b+j c-k d
\end{gathered}
$$

for $a+i b+j c+k d \in \mathbb{H}$. Clearly, $\varphi, \psi$ are $\mathbb{R}$-linear endomorphisms of the Euclidean vector space $\mathbb{H}$. We claim that $J \circ(\varphi \times \psi)=\psi \circ J$. To that end let $\zeta=a_{1}+$ $i b_{1}+j c_{1}+k d_{1} \in C$ and $v=a_{2}+i b_{2}+j c_{2}+k d_{2} \in V$. Then

$$
\begin{aligned}
\zeta v= & a_{1} a_{2}-b_{1} b_{2}-c_{1} c_{2}-d_{1} d_{2}+i\left(a_{1} b_{2}+b_{1} a_{2}+c_{1} d_{2}-d_{1} c_{2}\right) \\
& +j\left(a_{1} c_{2}-b_{1} d_{2}+c_{1} a_{2}+d_{1} b_{2}\right)+k\left(a_{1} d_{2}+b_{1} c_{2}-c_{1} b_{2}+d_{1} a_{2}\right) .
\end{aligned}
$$

Therefore,

$$
\begin{aligned}
\psi(\zeta v)= & -a_{1} a_{2}+b_{1} b_{2}+c_{1} c_{2}+d_{1} d_{2}+i\left(a_{1} b_{2}+b_{1} a_{2}+c_{1} d_{2}-d_{1} c_{2}\right) \\
& +j\left(a_{1} c_{2}-b_{1} d_{2}+c_{1} a_{2}+d_{1} b_{2}\right)+k\left(-a_{1} d_{2}-b_{1} c_{2}+c_{1} b_{2}-d_{1} a_{2}\right) .
\end{aligned}
$$


On the other side we get

$$
\begin{aligned}
\varphi(\zeta) \psi(v)= & \left(a_{1}-i b_{1}-j c_{1}+k d_{1}\right)\left(-a_{2}+i b_{2}+j c_{2}-k d_{2}\right) \\
= & -a_{1} a_{2}+b_{1} b_{2}+c_{1} c_{2}+d_{1} d_{2}+i\left(a_{1} b_{2}+b_{1} a_{2}+c_{1} d_{2}-d_{1} c_{2}\right) \\
& +j\left(a_{1} c_{2}-b_{1} d_{2}+c_{1} a_{2}+d_{1} b_{2}\right)+k\left(-a_{1} d_{2}-b_{1} c_{2}+c_{1} b_{2}-d_{1} a_{2}\right) \\
= & \psi(\zeta v) .
\end{aligned}
$$

Therefore, $m=(\varphi, \psi) \in M$, but $m \notin M^{\text {res }}$.

4.5 Isometric spheres via cocycles

For an element $g \in G^{\text {res }} \backslash G_{\infty}$, we give a characterization of the isometric sphere and its radius via a cocycle. Using the isometry $\beta: H \rightarrow D$ we find for the height function on $H$ the formula

$$
\mathrm{ht}^{H}(\zeta, v)=\operatorname{Re} \zeta-\frac{1}{2}|v|^{2}
$$

and for the Cygan metric $\left(z_{j}=\left(\zeta_{j}, v_{j}\right)\right)$

$\rho^{H}\left(z_{1}, z_{2}\right)=\left.\left|\frac{1}{2}\right| v_{1}\right|^{2}+\frac{1}{2}\left|v_{2}\right|^{2}+\left|\mathrm{ht}^{H}\left(z_{1}\right)-\mathrm{ht}^{H}\left(z_{2}\right)\right|+\operatorname{Im} \zeta_{1}-\operatorname{Im} \zeta_{2}-\left.\beta_{2}\left(v_{1}, v_{2}\right)\right|^{1 / 2}$

The basis point in $H$ is $o^{H}=(1,0)$. The horospherical coordinates of $z \in \bar{H}^{g} \backslash\{\infty\}$, $z=(\zeta, v)$, are

$$
\left(\mathrm{ht}^{H}(z), \operatorname{Im} \zeta, \frac{1}{\sqrt{2}} v\right)_{h} .
$$

In the following we derive a formula for isometric spheres which uses a cocycle.

Let $Z^{1}(C)$ act diagonally on $E$ and suppose that $\pi^{(1)}: E \rightarrow E / Z^{1}(C)$ is the canonical projection. Further set

$$
\pi_{H}^{(1)}:=\tau_{H} \circ \pi \circ\left(\pi^{(1)}\right)^{-1}:\left(E_{-}\left(\Psi_{2}\right) \cup E_{0}\left(\Psi_{2}\right)\right) / Z^{1}(C) \rightarrow \bar{H}^{g} .
$$

Recall the section $i_{H}$ of $\pi_{H}$ from (4.5) and set

$$
i_{H}^{(1)}:=\pi^{(1)} \circ i_{H}: \bar{H}^{g} \rightarrow E / Z^{1}(C) .
$$

Further recall the isomorphism $\varphi_{H}: G^{\text {res }} \rightarrow \mathrm{PU}\left(\Psi_{2}, C\right)$ from (4.6). For each $g \in G^{\text {res }}$ the map $\varphi_{H}(g): E \rightarrow E$ induces canonically a map $E / Z^{1}(C) \rightarrow E / Z^{1}(C)$, which we denote by $\varphi_{H}(g)$ as well. For all $g \in G^{\text {res }}$ the diagram

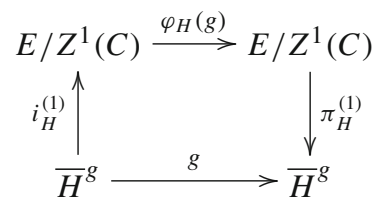


commutes, but the diagram

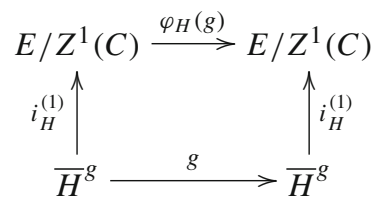

in general not. The second diagram gives rise to the cocycle

$$
j: G^{\mathrm{res}} \times \bar{H}^{g} \rightarrow C^{\times} / Z^{1}(C)
$$

defined by

$$
\varphi_{H}(g)\left(i_{H}^{(1)}(z)\right)=j(g, z) i_{H}^{(1)}(g z) \quad \forall g \in G^{\mathrm{res}} \forall z \in \bar{H}^{g} .
$$

Lemma 4.30 Let $g=n_{2} \sigma m a_{t} n_{1} \in G^{\mathrm{res}} \backslash G_{\infty}$. Then

$$
j\left(g^{-1}, \infty\right)=t^{1 / 2} \bmod Z^{1}(C) .
$$

Further, $R(g)=\left|j\left(g^{-1}, \infty\right)\right|^{-1 / 2}$.

Proof Suppose that $n_{1}=\left(\xi_{1}, w_{1}\right)$. From $n_{1}^{-1}=\left(-\xi_{1},-w_{1}\right)$ and $g^{-1}=n_{1}^{-1} \sigma a_{t} m n_{2}^{-1}$ it follows that

$$
g^{-1} \infty=n_{1}^{-1} \sigma \infty=n_{1}^{-1} 0=\left(-\xi_{1}+\frac{1}{4}\left|w_{1}\right|^{2},-\frac{1}{\sqrt{2}} w_{1}\right) .
$$

Further

$$
\varphi_{H}\left(g^{-1}\right)\left(i_{H}(\infty)\right)=\left(t^{1 / 2}, t^{1 / 2}\left(-\xi_{1}+\frac{1}{4}\left|w_{1}\right|^{2}\right),-\frac{1}{\sqrt{2}} t^{1 / 2} w_{1}\right) \quad \bmod Z^{1}(C) .
$$

Hence $j\left(g^{-1}, \infty\right)=t^{1 / 2} \bmod Z^{1}(C)$. Then $R(g)=t^{-1 / 4}=\left|j\left(g^{-1}, \infty\right)\right|^{-1 / 2}$.

Proposition 4.31 Let $z_{1}, z_{2} \in \bar{H}^{g} \backslash\{\infty\}, z_{j}=\left(\zeta_{j}, v_{j}\right)$. Then

$$
\rho^{H}\left(z_{1}, z_{2}\right)=\left|\Psi_{2}\left(i_{H}\left(z_{1}\right), i_{H}\left(z_{2}\right)\right)+2 \min \left(\mathrm{ht}^{H}\left(z_{1}\right), \mathrm{ht}^{H}\left(z_{2}\right)\right)\right|^{1 / 2} .
$$

Proof For all $k_{1}, k_{2} \in \mathbb{R}$ we have

$$
k_{1}+k_{2}-\left|k_{1}-k_{2}\right|=2 \min \left(k_{1}, k_{2}\right) .
$$

This and the definition of $\Psi_{2}$ show that

$$
\begin{aligned}
\mid & \Psi_{2}\left(i_{H}\left(z_{1}\right), i_{H}\left(z_{2}\right)\right)+2 \min \left(\mathrm{ht}^{H}\left(z_{1}\right), \mathrm{ht}^{H}\left(z_{2}\right)\right) \mid \\
= & \left|\Psi_{2}\left(\left(1, \zeta_{1}, v_{1}\right),\left(1, \zeta_{2}, v_{2}\right)\right)+\mathrm{ht}^{H}\left(z_{1}\right)+\mathrm{ht}^{H}\left(z_{2}\right)-\right| \mathrm{ht}^{H}\left(z_{1}\right)-\mathrm{ht}^{H}\left(z_{2}\right)|| \\
= & -\beta_{1}\left(1, \zeta_{2}\right)-\beta_{1}\left(\zeta_{1}, 1\right)+\beta_{2}\left(v_{1}, v_{2}\right)+\mathrm{ht}^{H}\left(z_{1}\right)+\mathrm{ht}^{H}\left(z_{2}\right)-\left|\mathrm{ht}^{H}\left(z_{1}\right)-\mathrm{ht}^{H}\left(z_{2}\right)\right| \mid \\
= & \zeta_{1}-\mathrm{ht}^{H}\left(z_{1}\right)+\bar{\zeta}_{2}-\mathrm{ht}^{H}\left(z_{2}\right)-\beta_{2}\left(v_{1}, v_{2}\right)+\left|\mathrm{ht}^{H}\left(z_{1}\right)-\mathrm{ht}^{H}\left(z_{2}\right)\right| \mid .
\end{aligned}
$$

Since

$$
\zeta_{1}-\mathrm{ht}^{H}\left(z_{1}\right)=\zeta_{1}-\operatorname{Re} \zeta_{1}+\frac{1}{2}\left|v_{1}\right|^{2}=\operatorname{Im} \zeta_{1}+\frac{1}{2}\left|v_{1}\right|^{2}
$$


and

$$
\bar{\zeta}_{2}-\mathrm{ht}^{H}\left(z_{2}\right)=\bar{\zeta}_{2}-\operatorname{Re} \zeta_{2}+\frac{1}{2}\left|v_{2}\right|^{2}=-\operatorname{Im} \zeta_{2}+\frac{1}{2}\left|v_{2}\right|^{2},
$$

it follows that

$$
\begin{aligned}
& \left|\Psi_{2}\left(i_{H}\left(z_{1}\right), i_{H}\left(z_{2}\right)\right)+2 \min \left(\mathrm{ht}^{H}\left(z_{1}\right), \mathrm{ht}^{H}\left(z_{2}\right)\right)\right| \\
& =\left.\left|\frac{1}{2}\right| v_{1}\right|^{2}+\operatorname{Im} \zeta_{1}+\frac{1}{2}\left|v_{2}\right|^{2}-\operatorname{Im} \zeta_{2}-\beta_{2}\left(v_{1}, v_{2}\right)+\left|\mathrm{ht}^{H}\left(z_{1}\right)-\mathrm{ht}^{H}\left(z_{2}\right)\right| \mid \\
& =\rho^{H}\left(z_{1}, z_{2}\right)^{2} .
\end{aligned}
$$

Since $\rho^{H}\left(z_{1}, z_{2}\right)$ is nonnegative, the claim follows.

For the proof of the following proposition we note that the map

$$
\Psi_{2}^{(1)}: \begin{cases}E / Z^{1}(C) \times E / Z^{1}(C) & \rightarrow C / Z^{1}(C) \\ \left(\left[z_{1}\right],\left[z_{2}\right]\right) & \mapsto\left[\Psi_{2}\left(z_{1}, z_{2}\right)\right]\end{cases}
$$

is well-defined. In particular, we have

$$
\left|\Psi_{2}^{(1)}\left(\left[z_{1}\right],\left[z_{2}\right]\right)\right|=\left|\Psi_{2}\left(z_{1}, z_{2}\right)\right|
$$

for all $z_{1}, z_{2} \in E$. Further, let

$$
\partial_{g} H:=\left\{\left.(\zeta, v) \in C \oplus V\left|\operatorname{Re}(\zeta)=\frac{1}{2}\right| v\right|^{2}\right\} \cup\{\infty\}
$$

denote the boundary of $H$ in $\bar{H}^{g}$.

Proposition 4.32 Let $g \in G^{\text {res }} \backslash G_{\infty}$ and $z \in \bar{H}^{g} \backslash\left\{\infty, g^{-1} \infty\right\}$. Then

$$
|j(g, z)|^{1 / 2}=\left|j\left(g^{-1}, \infty\right)\right|^{1 / 2} \rho^{H}\left(z, g^{-1} \infty\right)=\frac{\rho^{H}\left(z, g^{-1} \infty\right)}{R(g)} .
$$

Proof The second equality is proved by Lemma 4.30. For the proof of the first equality recall from Proposition 4.31 that

$$
\rho^{H}\left(z, g^{-1} \infty\right)=\left|\Psi_{2}\left(i_{H}(z), i_{H}\left(g^{-1} \infty\right)\right)+2 \min \left(\mathrm{ht}^{H}(z), \mathrm{ht}^{H}\left(g^{-1} \infty\right)\right)\right|^{1 / 2} .
$$

From $g^{-1} \infty \in \partial_{g} H \backslash\{\infty\}$ it follows that $h t\left(g^{-1} \infty\right)=0$. Hence

$$
\rho\left(z, g^{-1} \infty\right)^{2}=\left|\Psi_{2}\left(i_{H}(z), i_{H}\left(g^{-1} \infty\right)\right)\right| .
$$

Suppose that $g z=(\zeta, v)$. Then

$$
\begin{aligned}
\Psi_{2}\left(i_{H}(g z), i_{H}(\infty)\right) & =\Psi_{2}((1, g z),(0,1,0)) \\
& =-\beta_{1}(1,1)-\beta_{1}(\zeta, 0)+\beta_{2}(v, 0)=-1
\end{aligned}
$$


It follows that

$$
\begin{aligned}
\rho^{H}\left(z, g^{-1} \infty\right)^{2} & =\left|\Psi_{2}\left(i_{H}(z), i_{H}\left(g^{-1} \infty\right)\right)\right|=\left|\Psi_{2}^{(1)}\left(i_{H}^{(1)}(z), i_{H}^{(1)}\left(g^{-1} \infty\right)\right)\right| \\
& =\left|\Psi_{2}^{(1)}\left(i_{H}^{(1)}(z), j\left(g^{-1}, \infty\right) \varphi_{H}\left(g^{-1}\right) i_{H}^{(1)}(\infty)\right)\right| \\
& =\left|j\left(g^{-1}, \infty\right)\right|^{-1}\left|\Psi_{2}^{(1)}\left(i_{H}^{(1)}(z), \varphi_{H}\left(g^{-1}\right) i_{H}^{(1)}(\infty)\right)\right| \\
& =\left|j\left(g^{-1}, \infty\right)\right|^{-1}\left|\Psi_{2}^{(1)}\left(\varphi_{H}(g) i_{H}^{(1)}(z), i_{H}^{(1)}(\infty)\right)\right| \\
& =\left|j\left(g^{-1}, \infty\right)\right|^{-1}|j(g, z)|\left|\Psi_{2}\left(i_{H}(g z), i_{H}(\infty)\right)\right| \\
& =\left|j\left(g^{-1}, \infty\right)\right|^{-1}|j(g, z)| .
\end{aligned}
$$

This proves the claim.

Proposition 4.33 Let $g \in G^{\mathrm{res}} \backslash G_{\infty}$. Then

$$
\begin{aligned}
I(g) & =\{z \in H|| j(g, z) \mid=1\}, \\
\operatorname{ext} I(g) & =\{z \in H|| j(g, z) \mid>1\}, \\
\text { int } I(g) & =\{z \in H|| j(g, z) \mid<1\} .
\end{aligned}
$$

Proof This claim follows immediately from comparing Definition 3.9 and Proposition 4.32.

\subsection{A special instance of Theorem 3.18}

The most frequent appearance of isometric fundamental regions in the literature is for properly discontinuous subgroups $\Gamma$ of $G^{\text {res }}$ for which $\infty$ is an ordinary point and the stabilizer $\Gamma_{\infty}$ is trivial. We show that under these conditions $\Gamma$ is of type $(\mathrm{O})$ and $\Gamma \backslash \Gamma_{\infty}$ of type $(\mathrm{F})$. Theorem 3.18 implies the existence of an isometric fundamental region for $\Gamma$, which will be seen to actually be a fundamental domain.

Definition 4.34 Let $U$ be a subset of $\bar{H}^{g}$ and $\Gamma$ a subgroup of $G$. Then $\Gamma$ is said to act properly discontinuously on $U$ if for each compact subset $K$ of $U$, the set $K \cap g K$ is nonempty for only finitely many $g$ in $\Gamma$. The group $\Gamma$ is said to be properly discontinous if $\Gamma$ acts properly discontinuously on $H$.

Definition and Remark 4.35 Let $\Gamma$ be a properly discontinuous subgroup of $G$ and $z \in H$. The limit set $L(\Gamma)$ of $\Gamma$ is the set of accumulation points of the orbit $\Gamma z$. Since $\Gamma$ is properly discontinuous, $L(\Gamma)$ is a subset of $\partial_{g} H$. The combination of Propositions 2.9 and 1.4 in [9] shows that $L(\Gamma)$ is independent of the choice of $z$. The ordinary set or discontinuity set $\Omega(\Gamma)$ of $\Gamma$ is the complement of $L(\Gamma)$ in $\partial_{g} H$, hence $\Omega(\Gamma):=\partial_{g} H \backslash L(\Gamma)$.

Remark 4.36 Let $g, h \in G^{\text {res }}$ and $z \in \bar{H}^{g}$. Since $\varphi_{H}$ is a group isomorphism and $\varphi_{H}(g)$ is $C$-linear, it follows that

$$
\begin{aligned}
\varphi_{H}(g h)\left(i_{H}(z)\right) & =\varphi_{H}(g)\left(\varphi_{H}(h)\left(i_{H}(z)\right)\right) \\
& =\varphi_{H}(g)\left(j(h, z) i_{H}(h z)\right) \\
& =j(h, z) \varphi_{H}(g)\left(i_{H}(h z)\right) \\
& =j(h, z) j(g, h z) i_{H}(h g z) .
\end{aligned}
$$


Further,

$$
\varphi_{H}(g h)\left(i_{H}(z)\right)=j(g h, z) i_{H}(g h z) .
$$

Thus,

$$
j(g h, z)=j(h, z) j(g, h z) .
$$

The proof of the following lemma proceeds along the lines of [10, Section 17].

Lemma 4.37 Let $\Gamma$ be a properly discontinuous subgroup of $G^{\text {res }}$ such that $\Gamma_{\infty}=\{\mathrm{id}\}$ and $\infty \in \Omega(\Gamma)$. Then

(i) the set of radii of the isometric spheres of $\Gamma$ is bounded from above.

(ii) the number of isometric spheres with radius exceeding a given positive quantity is finite.

Proof We start by proving some relations between radii and distances of centers of isometric spheres. Let $g, h \in \Gamma \backslash \Gamma_{\infty}$ such that $g \neq h^{-1}$. The cocycle relation shows that

$$
j\left((g h)^{-1}, \infty\right)=j\left(g^{-1}, \infty\right) j\left(h^{-1}, g^{-1} \infty\right) .
$$

Proposition 4.32 yields

$$
\begin{aligned}
\left|j\left((g h)^{-1}, \infty\right)\right|^{-1 / 2} & =\left|j\left(g^{-1}, \infty\right)\right|^{-1 / 2}\left|j\left(h^{-1}, g^{-1} \infty\right)\right|^{-1 / 2} \\
& =\left|j\left(g^{-1}, \infty\right)\right|^{-1 / 2}|j(h, \infty)|^{-1 / 2} \rho^{H}\left(g^{-1} \infty, h \infty\right)^{-1} .
\end{aligned}
$$

Note that $g h \neq \mathrm{id}$ and therefore $g h \notin \Gamma_{\infty}$. Then Lemma 4.30 shows that

$$
R(g h)=\frac{R(g) R\left(h^{-1}\right)}{\rho^{H}\left(g^{-1} \infty, h \infty\right)} .
$$

Using the same arguments, we find

$$
j\left(g^{-1}, \infty\right)=j\left(h(g h)^{-1}, \infty\right)=j\left((g h)^{-1}, \infty\right) j\left(h,(g h)^{-1} \infty\right)
$$

and therefore

$$
\begin{aligned}
R(g) & =\left|j\left(g^{-1}, \infty\right)\right|^{-1 / 2}=\left|j\left((g h)^{-1}, \infty\right)\right|^{-1 / 2}\left|j\left(h,(g h)^{-1} \infty\right)\right|^{-1 / 2} \\
& =R(g h)\left|j\left(h,(g h)^{-1} \infty\right)\right|^{-1 / 2} .
\end{aligned}
$$

Proposition 4.32 shows that identity

$$
\left|j\left(h,(g h)^{-1} \infty\right)\right|^{1 / 2}=R(h)^{-1} \rho^{H}\left((g h)^{-1} \infty, h^{-1} \infty\right) .
$$

Because $R(h)=R\left(h^{-1}\right)$, it follows from (4.8) to (4.10) that

$$
\begin{aligned}
\rho^{H}\left((g h)^{-1} \infty, h^{-1} \infty\right) & =\left|j\left(h,(g h)^{-1} \infty\right)\right|^{1 / 2} R(h)=\frac{R(g h) R(h)}{R(g)} \\
& =\frac{R(h)^{2}}{\rho^{H}\left(g^{-1} \infty, h \infty\right)} .
\end{aligned}
$$

Since $\Gamma$ is properly discontinuous and $\infty \in \Omega(\Gamma)$, [9, Proposition 8.5] shows that there exists an open neighborhood $U$ of $\infty$ in $\bar{H}^{g}$ such that $\left(\Gamma \backslash \Gamma_{\infty}\right) \subseteq \bar{H}^{g} \backslash U$. Since $\bar{H}^{g} \backslash U$ is compact, we find $m>0$ such that

$$
\rho^{H}(a \infty, b \infty)<m
$$


for all $a, b \in \Gamma \backslash \Gamma_{\infty}$. Then (4.11) shows that

$$
R(h)^{2}=\rho^{H}\left((g h)^{-1} \infty, h^{-1} \infty\right) \rho^{H}\left(g^{-1} \infty, h \infty\right)<m^{2} .
$$

Thus, for each $a \in \Gamma \backslash \Gamma_{\infty}$ we have $R(a)<m$, which proves (i).

Now let $k>0$ and suppose that there are $a, b \in \Gamma \backslash \Gamma_{\infty}$ such that $I(a) \neq I\left(b^{-1}\right)$ and $R(a), R(b)>k$. Since $a \neq b^{-1}$, (4.8) shows in combination with (4.12) that

$$
\rho^{H}\left(a^{-1} \infty, b \infty\right)=\frac{R(a) R(b)}{R(a b)}>\frac{k^{2}}{m} .
$$

This means that the distance between the centers of isometric spheres whose radii exceed $k$ is bounded from below by $k^{2} / \mathrm{m}$. The centers of all these isometric spheres are contained in the compact set $\partial_{g} H \backslash U$, which is bounded in $C \times V$ and hence also w.r.t. $\rho^{H}$. It follows that there are only finitely many spheres with radius exceeding $k$. This proves (ii).

Proposition 4.38 Suppose that $\Gamma$ is a properly discontinuous subgroup of $G^{\text {res }}$ such that $\Gamma_{\infty}=\{\mathrm{id}\}$ and $\infty \in \Omega(\Gamma)$. Then $\Gamma$ is of type $(O)$ and $\Gamma \backslash \Gamma_{\infty}$ of type $(F)$. Moreover,

$$
\mathcal{F}:=\bigcap_{g \in \Gamma \backslash \Gamma_{\infty}} \operatorname{ext} I(g)
$$

is a fundamental domain for $\Gamma$ in $H$.

Proof For each $z \in H$, the map $\rho^{H}(\cdot, z): H \rightarrow \mathbb{R}$ is continuous. Therefore, each $\rho^{H}$-ball is open in $H$. Lemma 4.37(ii) implies that the set \{int $\left.I(g) \mid g \in \Gamma \backslash \Gamma_{\infty}\right\}$ is locally finite. Then Remark 3.19 shows that $\Gamma$ is of type (O). Note that here the subgroup $\left\langle\Gamma \backslash \Gamma_{\infty}\right\rangle$ of $\Gamma$ which is generated by $\Gamma \backslash \Gamma_{\infty}$ is exactly $\Gamma$. Let $z \in H$. Lemma 5 before Theorem 5.3.4 in [21] states that $\Gamma z$ is a closed subset of $H$. Since $\infty \in \Omega(\Gamma)$, Proposition 8.5 in [9] shows that we find an open neighborhood $U$ of $\infty$ in $\bar{H}^{g}$ such that $\Gamma z \subseteq \bar{H}^{g} \backslash U$. Now $\bar{H}^{g} \backslash U$ is compact and therefore $\Gamma z$ is so. The height function is continuous, which shows that the maximum of $\{h t(g z) \mid g \in \Gamma\}$ exists. Thus, $\Gamma \backslash \Gamma_{\infty}$ is of type $(\mathrm{F})$. By Theorem 3.18, $\mathcal{F}$ is a fundamental region for $\Gamma$ in $H$. By Lemma 4.37(i) the radii of the isometric spheres of $\Gamma$ are uniformly bounded from above. Hence there is $R>0$ such that the arc-connected set $\left\{z \in H \mid \mathrm{ht}^{H}(z)=R\right\}$ is contained in $\mathcal{F}$. Let $w_{1}, w_{2} \in \mathcal{F}$. Then $w_{1}+\left[0, R-\mathrm{ht}^{H}\left(w_{1}\right)\right]$ and $w_{2}+\left[0, R-\mathrm{ht}^{H}\left(w_{2}\right)\right]$ are contained in $\mathcal{F}$ by Lemma 3.11(v). Hence, there is an arc in $\mathcal{F}$ from $w_{1}$ to $w_{2}$. This shows that $\mathcal{F}$ is arc-connected, and thus connected.

\subsection{Isometric spheres and isometric fundamental regions in the literature}

For real hyperbolic spaces, definitions of isometric spheres are given at several places, e. g., the original definition of Ford in [10] for the plane, in [13] for the upper half plane model and the disk (ball) model of two-dimensional real hyperbolic space, in [17] for threedimensional space and in [2] (or, earlier, in [1]) for arbitrary dimensions. The definition for the upper half plane model is not equivalent to that in the disk model (see [17]). Ford's definition is that for the upper half plane model. His definition has been directly generalized to higher dimensions. Ford and Apanasov show the existence of isometric fundamental regions for a huge class of groups.

For complex hyperbolic spaces, the (to the knowledge of the author) only existing definitions of isometric spheres are given by Parker in [18], Goldman in [11], and Kamiya in [12]. Kamiya also discusses the existence of isometric fundamental regions for certain groups.

For quaternionic hyperbolic spaces, an investigation of isometric fundamental regions does not seem to exist. A definition of isometric spheres is provided by [14]. 
In this final section, we discuss the relation of the existing definitions of isometric spheres (for real hyperbolic space only exemplarily) to our definition of isometric spheres, and we compare the existing statements on the existence of isometric fundamental regions to Theorem 3.18.

\subsection{Real hyperbolic spaces}

Ford [10] shows the existence of isometric fundamental regions for groups of isometries acting on real hyperbolic plane (see [10, Theorems 15 and 22 in Ch. III]). Ford's definition of fundamental region is not equivalent to our definition. In fact, each fundamental region in sense of our definition is a fundamental region in the sense of Ford, but not the other way round. In particular, the translates of a fundamental region in sense of Ford are not required to cover the whole space. For this reason the hypothesis of [10, Theorems 15, 22] are weaker than that of Theorem 3.18. However, the following discussion shows that the definition of isometric spheres in [10] is subsumed by our definition.

In [2], the model

$$
D^{\prime}=\{(t, Z) \in \mathbb{R} \times \mathfrak{z} \mid t>0\}
$$

of real hyperbolic space is used, and the isometric sphere for an element $g \in G^{\text {res }}$, acting on $D^{\prime}$, is defined as

$$
I(g):=\left\{z \in D^{\prime}|| g^{\prime}(z) \mid=1\right\} .
$$

More precisely, Apanasov (as all other references) uses the coordinates in the order $\mathfrak{z} \times \mathbb{R}$. In particular, his model space for two-dimensional real hyperbolic space is the upper half plane, whereas $D^{\prime}$ is the right half plane. Clearly, this difference has no affect on his definition of isometric sphere. Lemma 4.39 below will show that this definition of isometric spheres is subsumed by our definition.

The space $D^{\prime}$ is the symmetric space in Sect. 2.4 constructed from the abelian $H$-type algebra $\mathfrak{n}=(\mathfrak{n},\{0\})=(\mathfrak{z},\{0\})$. In Sects. 4-4.6 we had to work with the ordered decomposition $(\{0\}, \mathfrak{v})=(\{0\}, \mathfrak{n})$ of $\mathfrak{n}$. Hence we used the symmetric space

$$
D=\left\{(u, X) \in \mathbb{R} \times\left.\mathfrak{v}\left|u>\frac{1}{4}\right| X\right|^{2}\right\},
$$

which is isometric to $D^{\prime}$. According to [7], the isometry from $D^{\prime}$ to $D$ is

$$
v:\left\{\begin{aligned}
D^{\prime} & \rightarrow D \\
(t, Z) & \mapsto\left(t^{2}+|Z|^{2}, 2 Z\right) .
\end{aligned}\right.
$$

Then the action of an isometry $g \in G^{\text {res }}$ on $D^{\prime}$ is given by $v_{G}(g):=v^{-1} \circ g \circ v$. Recall the isometry $\beta: H \rightarrow D$ from Sect. 4.4.1.

Lemma 4.39 Let $z \in D^{\prime}$ and $g \in G^{r e s} \backslash G_{\infty}^{r e s}$. Then

$$
\left|j\left(g, \beta^{-1} \circ v(z)\right)\right|^{-1}=\left|v_{G}(g)^{\prime}(z)\right|
$$


Proof Let $z=(t, Z) \in D^{\prime}$ and $g=n_{2} \sigma m a_{s} n_{1} \in G^{\text {res }} \backslash G_{\infty}^{\text {res }}$ with $n_{j}=\left(1, w_{j}\right), j=1,2$, and $m=(\mathrm{id}, \psi)$. At first we calculate the value of $\left|j\left(g, \beta^{-1} \circ v(z)\right)\right|$. We have

$$
\begin{aligned}
\varphi_{H}(g) i_{H}\left(\beta^{-1} \circ v(z)\right) & =\varphi_{H}(g)\left(1, t^{2}+|Z|^{2}, \sqrt{2} Z\right) \\
& =\varphi_{H}\left(n_{2}\right) \varphi_{H}(\sigma) \varphi_{H}(m) \varphi_{H}\left(a_{s}\right) \varphi_{H}\left(n_{1}\right)\left(1, t^{2}+|Z|^{2}, \sqrt{2} Z\right) \\
& =\left(s^{1 / 2}\left(\frac{1}{4}\left|w_{1}\right|^{2}+t^{2}+|Z|^{2}+\beta_{2}\left(Z, w_{1}\right)\right), *, *\right) .
\end{aligned}
$$

Thus

$$
\left|j\left(g, \beta^{-1} \circ v(z)\right)\right|=\left.s^{1 / 2}\left|\frac{1}{4}\right| w_{1}\right|^{2}+t^{2}+|Z|^{2}+\beta_{2}\left(Z, w_{1}\right) \mid .
$$

Note that $Z$ and $\beta_{2}\left(Z, w_{1}\right)$ have to be seen as element of $D$ (not of $\left.D^{\prime}\right)$. Hence $\beta_{2}\left(Z, w_{1}\right) \in \mathbb{R}$. Therefore, $\beta_{2}\left(Z, w_{1}\right)=\left\langle Z, w_{1}\right\rangle$ and further

$$
\left|j\left(g, \beta^{-1} \circ v(z)\right)\right|=s^{1 / 2}\left|t^{2}+\right| \frac{1}{2} w_{1}+\left.\left.Z\right|^{2}\left|=s^{1 / 2}\right|\left(t, \frac{1}{2} w_{1}+Z\right)\right|^{2} .
$$

Let $(u, W) \in \mathbb{R} \times \mathfrak{z}$. For the derivative $v_{G}(g)^{\prime}(z)$ we find

$$
v_{G}(g)^{\prime}(z)(u, W)=-s^{-1 / 2}\left(t, \psi\left(\frac{1}{2} w_{1}+Z\right)\right)^{-1}(u, \psi(W))\left(t, \psi\left(\frac{1}{2} w_{1}+Z\right)\right)^{-1} .
$$

Then

$$
\begin{aligned}
\left|v_{G}(g)^{\prime}(z)(u, W)\right|= & s^{-1 / 2}\left|\left(t, \psi\left(\frac{1}{2} w_{1}+Z\right)\right)\right|^{-1} \cdot|(u, \psi(W))| \\
& \cdot\left|\left(t, \psi\left(\frac{1}{2} w_{1}+Z\right)\right)\right|^{-1} \\
= & s^{-1 / 2}\left|\left(t, \frac{1}{2} w_{1}+Z\right)\right|^{-2}|(u, W)| .
\end{aligned}
$$

Thus,

$$
\left|v_{G}(g)^{\prime}(z)\right|=s^{-1 / 2}\left|\left(t, \frac{1}{2} w_{1}+Z\right)\right|^{-2}=\left|j\left(g, \beta^{-1} \circ v(z)\right)\right|^{-1} .
$$

This completes the proof.

Lemma 4.39 and Proposition 4.33 immediately imply the following characterization of exteriors of isometric spheres.

Proposition 4.40 Let $g \in G^{r e s} \backslash G_{\infty}^{r e s}$. Then

$$
\operatorname{ext} I(g)=\left\{z \in D^{\prime}|| v_{G}(g)^{\prime}(z) \mid<1\right\} .
$$

Hence [2, Theorem 2.30] is a special case of Proposition 4.38. Lemma 2.31 in [2] states an extension of Theorem 2.30 for subgroups $\Gamma$ of $G^{\text {res }}$ with $\Gamma_{\infty} \neq\{$ id $\}$. Unfortunately, the hypotheses of [2, Lemma 2.31] are not completely stated, for which reason we cannot compare this lemma with Theorem 3.18. 


\subsection{Complex hyperbolic spaces}

The isometric spheres for isometries of complex hyperbolic spaces in [12] are identical to those in [11]. Kamiya uses the model $H$ and defines the Cygan metric by formula (4.7). Recall the map $\varphi_{H}$ from Sect. 4.4.6. Let $f \in \operatorname{PU}(1, n ; \mathbb{C})=\operatorname{PU}\left(\Psi_{2}, \mathbb{C}\right)$ such that $\varphi_{H}^{-1}(f)$ does not fix $\infty$. Further suppose that $\left(a_{i j}\right)_{i, j=1, \ldots, n+1}$ is a matrix representative of $f$. Then Kamiya defines the isometric sphere of $f$ to be the set

$$
I(f):=\left\{z \in H \mid \rho\left(z, \varphi_{H}^{-1}\left(f^{-1}\right) \infty\right)=R_{f}\right\}
$$

where $R_{f}:=\left|a_{12}\right|^{-1 / 2}$. One easily proves that this definition does not depend on the choice of the matrix representative. The following lemma shows that our definition of isometric spheres covers this one.

Lemma 4.41 Suppose that $f=\left(a_{i j}\right)_{i, j=1, \ldots, n+1} \in \mathrm{PU}(1, n ; \mathbb{C})$ with $\varphi_{H}^{-1}(f)(\infty) \neq \infty$. Then $R_{f}=R\left(\varphi_{H}^{-1}(f)\right)$.

Proof Set $g:=\varphi_{H}^{-1}(f)$. We have

$$
f\left(\begin{array}{l}
0 \\
1 \\
0
\end{array}\right)=\left(\begin{array}{c}
a_{12} \\
\vdots \\
a_{n+1,2}
\end{array}\right) .
$$

Therefore,

$$
|j(g, \infty)|=\left|a_{12}\right| .
$$

Since $\left|j\left(g^{-1}, \infty\right)\right|=|j(g, \infty)|$, Lemma 4.30 shows that $R(g)=\left|a_{12}\right|^{-1 / 2}$.

[12, Theorem 3.1] states the existence of isometric fundamental domains for discrete subgroups $\Gamma$ of $G^{\text {res }}$ for which, after possible conjugation of $\Gamma$, we have $\infty \in \Omega(\Gamma)$ and $\Gamma_{\infty}=\{\mathrm{id}\}$. By [21, Theorem 5.3.5], $\Gamma$ is discrete if and only if $\Gamma$ is properly discontinuous. Therefore, Kamiya's Theorem is a special case of Proposition 4.38.

In [18], Parker uses a section of the projection map from $E \backslash\{0\}$ to horospherical coordinates which is reminiscent of the ball model. Therefore, we expect that, as in the real case, this definition is not equivalent to the definition from [12].

\subsection{Quaternionic hyperbolic spaces}

In [14], Kim and Parker propose a definition of isometric spheres for isometries in $G^{\text {res }} \backslash G_{\infty}^{\text {res }}$ of quaternionic hyperbolic space. They use the model $H$ and horospherical coordinates for the definition.

With the bijection

$$
\left\{\begin{array}{l}
\mathfrak{z} \times \mathfrak{v} \rightarrow \mathfrak{z} \times \mathfrak{v} \\
(Z, X) \mapsto\left(Z, \frac{1}{2} X\right),
\end{array}\right.
$$

our Heisenberg group $N$ and our Cygan metric is transferred into their one. After shuffling coordinates, they characterize (see [14, Proposition 4.3]) the isometric sphere $I(g)$ of an element $g=\left(a_{i j}\right)_{i, j=1, \ldots, n+1}$ in $\operatorname{PSp}(n, 1 ; \mathbb{H})$ with $\varphi_{H}^{-1}(g)(\infty) \neq \infty$ as

$$
I(g)=\left\{\left.z \in H\left|\rho\left(z, \varphi_{H}^{-1}\left(g^{-1}\right) \infty\right)=\sqrt{2} \cdot\right| a_{12}\right|^{1 / 2}\right\} .
$$


They use a slightly different indefinite form on $E$ for the definition of the hyperbolic space. Despite this difference we can apply the calculation in Sect. 4.9 we see that our definition of isometric sphere provides $\left|a_{12}\right|^{1 / 2}$ as radius. The factor $\sqrt{2}$ in [14] is due to the factor $\frac{1}{2}$ in their choice of the section of the projection from $E_{-}\left(\Psi_{2}\right)$ to $H$.

Acknowledgments The author was partially supported by the International Research Training Group 1133 "Geometry and Analysis of Symmetries", the Sonderforschungsbereich/Transregio 45 "Periods, moduli spaces and arithmetic of algebraic varieties", and the Max-Planck-Institut für Mathematik in Bonn.

Open Access This article is distributed under the terms of the Creative Commons Attribution Noncommercial License which permits any noncommercial use, distribution, and reproduction in any medium, provided the original author(s) and source are credited.

\section{References}

1. Apanasov, B.: Discrete groups in space and uniformization problems. Mathematics and its Applications (Soviet Series), vol. 40. Kluwer, Dordrecht (translated and revised from the 1983 Russian original) (1991)

2. Apanasov, B.: Conformal Geometry of Discrete Groups and Manifolds, de Gruyter Expositions in Mathematics, vol. 32. Walter de Gruyter, Berlin (2000)

3. Borel, A., Ji, L.: Compactifications of Symmetric and Locally Symmetric Spaces. Mathematics: Theory \& Applications. Birkhäuser Boston, Boston (2006)

4. Bourbaki, N.: Algebra I. Chapters 1-3. Elements of Mathematics (Berlin). Springer, Berlin (translated from the French, Reprint of the 1989 English translation) (1998)

5. Chen, S., Greenberg, L.: Hyperbolic spaces. In: Ahlfors, L.V., Kra, I., Maskit, B., Nirenberg, L. (eds.) Contributions to Analysis (a collection of papers dedicated to Lipman Bers), pp. 49-87. Academic Press, New York (1974)

6. Cowling, M., Dooley, A., Korányi, A., Ricci, F.: H-type groups and Iwasawa decompositions. Adv. Math. 87(1), 1-41 (1991)

7. Cowling, M., Dooley, A., Korányi, A., Ricci, F.: An approach to symmetric spaces of rank one via groups of Heisenberg type. J. Geom. Anal. 8(2), 199-237 (1998)

8. Eberlein, P.: Geometry of Nonpositively Curved Manifolds. Chicago Lectures in Mathematics. University of Chicago Press, Chicago (1996)

9. Eberlein, P., O'Neill, B.: Visibility manifolds. Pac. J. Math. 46, 45-109 (1973)

10. Ford, L.: Automorphic Functions. Chelsea, New York (1972)

11. Goldman, W.: Complex Hyperbolic Geometry. Oxford Mathematical Monographs. The Clarendon Press, Oxford University Press, Oxford Science Publications, New York (1999)

12. Kamiya, S.: Generalized isometric spheres and fundamental domains for discrete subgroups of PU $(1, n ;$ C). Proc. Jpn. Acad. Ser. A Math. Sci. 79(5), 105-109 (2003)

13. Katok, S.: Fuchsian Groups. Chicago Lectures in Mathematics. University of Chicago Press, Chicago (1992)

14. Kim, I., Parker, J.: Geometry of quaternionic hyperbolic manifolds. Math. Proc. Camb. Philos. Soc. 135(2), 291-320 (2003)

15. Korányi, A., Ricci, F.: A unified approach to compact symmetric spaces of rank one. To appear in Colloquium Math

16. Korányi, A., Ricci, F.: A classification-free construction of rank-one symmetric spaces. Bull. Kerala Math. Assoc. (2005) (Special Issue), 73-88 (2007)

17. Marden, A.: Outer Circles. An Introduction to Hyperbolic 3-Manifolds. Cambridge University Press, Cambridge (2007)

18. Parker, J.: On Ford isometric spheres in complex hyperbolic space. Math. Proc. Camb. Philos. Soc. 115(3), 501-512 (1994)

19. Pohl, A.: Symbolic dynamics for the geodesic flow on locally symmetric good orbifolds of rank one. Dissertation (2009)

20. von Querenburg, B.: Mengentheoretische Topologie, 2nd edn. Springer, Berlin (1979) (Hochschultext)

21. Ratcliffe, J.: Foundations of Hyperbolic Manifolds, Graduate Texts in Mathematics, vol. 149. 2nd edn. Springer, New York (2006) 